Supporting Information

\title{
Transition-Metal- and Light-Free Generation of an Iminyl Radical: Facile Approach to Oxindoles and Isoquinolinediones with a Quaternary Carbon Center via Cyanoalkylarylation
}

Lanjun Su, ${ }^{a}$ Pan Xue, ${ }^{b}$ Xin Zhu, ${ }^{b}$ Huan Sun ${ }^{\star a}$, Jikai Liu*a, Chengming Wang ${ }^{* b}$

a. School of Pharmaceutical Sciences

South-Central University for Nationalities

Wuhan, 430074, China

Email: liujikai@mail.scuec.edu.cn, sunh15@163.com

b. Department of Chemistry, College of Chemistry and Materials Science Jinan University

Guangzhou, 511443, China

Email: cmwang2019@jnu.edu.cn

Table of Contents:

\begin{tabular}{lr}
\hline General Methods and Materials & S2 \\
Conditions Screening & S3 \\
General Procedure & S5 \\
Characterization of Products & S6 \\
Diversity of the Products & S17 \\
Mechanism Study & S19 \\
References & S23 \\
NMR Spectra Images of Products & S24 \\
\hline
\end{tabular}




\section{General Methods and Materials:}

Unless specified, all reactions were carried out under a nitrogen atmosphere with dry solvents under anhydrous conditions. $\mathrm{N}$-arylacrylamide and cyclobutanone oxime ester starting materials were synthesized according to a previous literature. ${ }^{1,2}$ DMSO (super dry, 99.7\%) was purchased from J\&K; all other reagents were purchased and used without further purification unless specified otherwise. Solvents for chromatography were technical grade and distilled prior to use. Flash chromatography was performed using 200-300 mesh silica gel with the indicated solvent system according to standard techniques. Analytical thin-layer chromatography (TLC) was performed on pre-coated, glass-backed silica gel plates. Visualization of the developed chromatogram was performed by UV absorbance $(254 \mathrm{~nm})$. The heat source was used oil bath. ${ }^{1} \mathrm{H}$ NMR and ${ }^{13} \mathrm{C}$ NMR data were recorded on Bruker $300 \mathrm{M}$ nuclear resonance spectrometers unless otherwise specified, respectively.

Chemical shifts $(\delta)$ in ppm are reported as quoted relative to the residual signals of chloroform ( ${ }^{1} \mathrm{H} 7.26 \mathrm{ppm}$ or $\left.{ }^{13} \mathrm{C} 77.16 \mathrm{ppm}\right)$. Multiplicities are described as: $\mathrm{s}$ (singlet), brs (broad singlet), d (doublet), t (triplet), q (quartet), $m$ (multiplet); and coupling constants $(J)$ are reported in Hertz $(\mathrm{Hz}) .{ }^{13} \mathrm{C}$ NMR spectra were recorded with total proton decoupling. High resolution mass spectrometry (HRMS) analysis was performed using electrospray ionization (ESI) with a quadrupole-time of flight (QTOF) mass analyzer. HRMS (ESI) analysis was performed by The Analytical Instrumentation Center at College of Chemistry and Materials Science, Jinan University, and (HRMS) data were reported with ion mass/charge $(\mathrm{m} / \mathrm{z})$ ratios as values in atomic mass units. 


\section{Conditions Screening a}

\begin{tabular}{|c|c|c|c|c|}
\hline Entry & Solvent & Temperature & Time & Yield \\
\hline 1 & THF & $110^{\circ} \mathrm{C}$ & $24 \mathrm{~h}$ & $54 \%$ \\
\hline 2 & 1,4-dioxane & $110^{\circ} \mathrm{C}$ & $24 \mathrm{~h}$ & $53 \%$ \\
\hline 3 & $\mathrm{MeCN}$ & $110^{\circ} \mathrm{C}$ & $24 \mathrm{~h}$ & $60 \%$ \\
\hline 4 & Toluene & $110^{\circ} \mathrm{C}$ & $24 \mathrm{~h}$ & $30 \%$ \\
\hline 5 & DCE & $110^{\circ} \mathrm{C}$ & $24 \mathrm{~h}$ & $55 \%$ \\
\hline 6 & DMF & $110^{\circ} \mathrm{C}$ & $24 \mathrm{~h}$ & $46 \%$ \\
\hline 7 & $n-\mathrm{Bu}_{2} \mathrm{O}$ & $110^{\circ} \mathrm{C}$ & $24 \mathrm{~h}$ & $26 \%$ \\
\hline 8 & DMSO & $110^{\circ} \mathrm{C}$ & $24 \mathrm{~h}$ & $61 \%$ \\
\hline 9 & MTBE & $110^{\circ} \mathrm{C}$ & $24 \mathrm{~h}$ & $34 \%$ \\
\hline 10 & $i-\mathrm{PrOH}$ & $110^{\circ} \mathrm{C}$ & $24 \mathrm{~h}$ & $52 \%$ \\
\hline 11 & $\mathrm{MeOH}$ & $110^{\circ} \mathrm{C}$ & $24 \mathrm{~h}$ & $31 \%$ \\
\hline 12 & DME & $110^{\circ} \mathrm{C}$ & $24 \mathrm{~h}$ & $44 \%$ \\
\hline 13 & DMSO & $25^{\circ} \mathrm{C}$ & $24 \mathrm{~h}$ & $0 \%$ \\
\hline 14 & DMSO & $40^{\circ} \mathrm{C}$ & $24 \mathrm{~h}$ & $0 \%$ \\
\hline 15 & DMSO & $60{ }^{\circ} \mathrm{C}$ & $24 \mathrm{~h}$ & trace \\
\hline 16 & DMSO & $80^{\circ} \mathrm{C}$ & $24 \mathrm{~h}$ & $<5 \%$ \\
\hline 17 & DMSO & $100{ }^{\circ} \mathrm{C}$ & $24 \mathrm{~h}$ & $32 \%$ \\
\hline 18 & DMSO & $120^{\circ} \mathrm{C}$ & $24 \mathrm{~h}$ & $63 \%$ \\
\hline 19 & DMSO & $110{ }^{\circ} \mathrm{C}$ & $4 \mathrm{~h}$ & $39 \%$ \\
\hline 20 & DMSO & $110^{\circ} \mathrm{C}$ & $6 \mathrm{~h}$ & $43 \%$ \\
\hline 21 & DMSO & $110^{\circ} \mathrm{C}$ & $12 \mathrm{~h}$ & $55 \%$ \\
\hline 22 & DMSO & $110^{\circ} \mathrm{C}$ & $30 \mathrm{~h}$ & $59 \%$ \\
\hline $23^{b}$ & DMSO & $110^{\circ} \mathrm{C}$ & $24 \mathrm{~h}$ & $47 \%$ \\
\hline $24^{c}$ & DMSO & $110^{\circ} \mathrm{C}$ & $24 \mathrm{~h}$ & $59 \%$ \\
\hline $25^{d}$ & DMSO & $110{ }^{\circ} \mathrm{C}$ & $24 \mathrm{~h}$ & $56 \%$ \\
\hline $26^{e}$ & DMSO & $110{ }^{\circ} \mathrm{C}$ & $24 \mathrm{~h}$ & $45 \%$ \\
\hline $27^{f}$ & DMSO & $110^{\circ} \mathrm{C}$ & $24 \mathrm{~h}$ & $43 \%$ \\
\hline $28^{g}$ & DMSO & $110{ }^{\circ} \mathrm{C}$ & $24 \mathrm{~h}$ & $50 \%$ \\
\hline $29^{h}$ & DMSO & $110^{\circ} \mathrm{C}$ & $24 \mathrm{~h}$ & $56 \%$ \\
\hline $30^{i}$ & DMSO & $110{ }^{\circ} \mathrm{C}$ & $24 \mathrm{~h}$ & $66 \%$ \\
\hline $31^{j}$ & DMSO & $110^{\circ} \mathrm{C}$ & $24 \mathrm{~h}$ & $61 \%$ \\
\hline
\end{tabular}

${ }^{a}$ Reaction on a 0.25 mmol scale, using 1 a (1.0 quiv.), 2 a (1.5 equiv.), solvent $(0.5 \mathrm{M}), 110^{\circ} \mathrm{C}$, under $\mathrm{N}_{2}, 24 \mathrm{~h},{ }^{1} \mathrm{H}$ NMR yield;

${ }^{b}$ DMSO (1.0 M); 
${ }^{c} \mathrm{DMSO}(0.8 \mathrm{M})$;

${ }^{d} \operatorname{DMSO}(0.3 \mathrm{M})$;

${ }^{e} \operatorname{DMSO}(0.2 \mathrm{M})$;

${ }^{f} \mathrm{DMSO}(0.1 \mathrm{M})$;

${ }^{g}$ 1a (1.0 equiv.), 2 a (1.0 equiv.);

${ }^{h}$ 1a (1.2 equiv.), 2 a (1.0 equiv.);

i 1 a (1.5 equiv.), 2a (1.0 equiv.);

${ }^{j} 1 \mathrm{a}$ (2.0 equiv.), $2 \mathrm{a}$ (1.0 equiv.).

Note:

$\mathrm{DCE}=1,2$-dichloroethane; $\mathrm{MTBE}=$ Methyl tert-butyl ether; $\mathrm{DME}=1$,2-Dimethoxyethane 


\section{General Procedure for the Radical Cyclization Reaction}<smiles>[R]C(=C)C(=O)N([R])c1cc[R1]([H])cc1</smiles>

1<smiles>[R]C1CC(=NOC(C)(O)O[Na])C1</smiles>

2

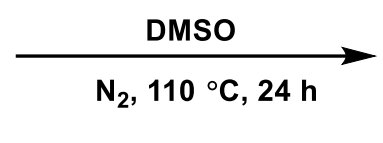

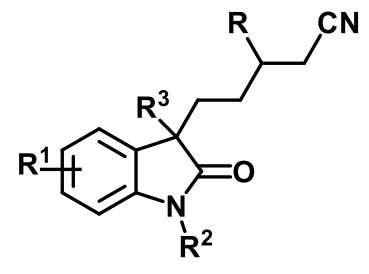

3

General procedure A: $N$-arylacrylamide $1(0.375 \mathrm{mmol}, 1.5$ equiv.) and DMSO (0.5 $\mathrm{mL}$ ) were weighed into a Schlenk tube. The reaction vessel was capped and subjected to three vacuum-purge/nitrogen-flush cycles. Then O-acyl oximes 2 (0.25 mmol, 1.0 equiv.) was added through the side-arm by syringe. The reaction was stirred under nitrogen at $110{ }^{\circ} \mathrm{C}$ for $24 \mathrm{~h}$. After reaction, the reaction was cooled to room temperature. Water $(20 \mathrm{~mL})$ was added, and the mixture was extracted with EtOAc, washed with brine, dried over $\mathrm{MgSO}_{4}$, and the organic extracts concentrated in vacuo. The residue was purified by silica gel flash chromatography using petroleum ether/EtOAc (20:1 to 5:1) to afford the desired product 3 .<smiles>[R][X]C1CCCCC1C(=O)N(C)C(=O)C(=C)C</smiles>

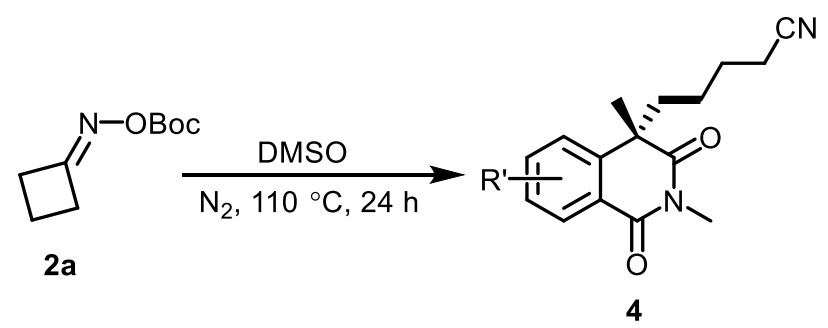

General procedure B: $N$-acrylamide $1(0.375 \mathrm{mmol}, 1.5$ equiv. $)$ and DMSO $(0.5 \mathrm{~mL})$ were added into a Schlenk tube. The reaction vessel was capped and subjected to three vacuum-purge/nitrogen-flush cycles. Then cyclobutanone oxime ester $2 \mathrm{a}$ (0.25 mmol, 1.0 equiv.) was added through the side-arm by syringe. The reaction was stirred under nitrogen at $110{ }^{\circ} \mathrm{C}$ for $24 \mathrm{~h}$. After reaction, the reaction was cooled to room temperature. Water $(20 \mathrm{~mL})$ was added, and the mixture was extracted with EtOAc, washed with brine, dried over $\mathrm{MgSO}_{4}$, and the organic extracts concentrated in vacuo. The residue was purified by silica gel flash chromatography using petroleum ether/EtOAc (20:1 to 5:1) to afford the desired product 4.

\section{Scale-up reaction $(1 \mathrm{mmol})$}

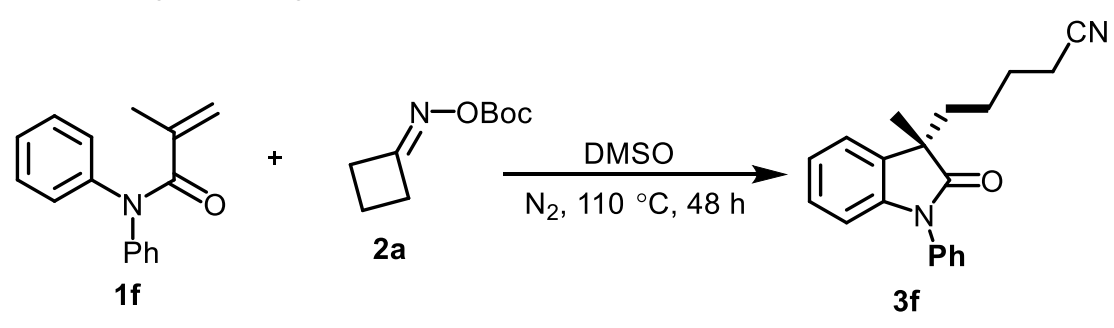


$\mathrm{N}$-phenylacrylamide $1 \mathrm{f}$ ( $356.0 \mathrm{mg}, 1.5 \mathrm{mmol}, 1.5$ equiv.) and DMSO (2.0 mL) were added into a Schlenk tube. The reaction vessel was capped and subjected to three vacuum-purge/nitrogen-flush cycles. Then O-acyl oximes 2a (185.2, $1.0 \mathrm{mmol}, 1.0$ equiv.) was added through the side-arm by syringe. The reaction was stirred under nitrogen at $110{ }^{\circ} \mathrm{C}$ for $48 \mathrm{~h}$. After reaction, the reaction was cooled to room temperature. Water $(20 \mathrm{~mL})$ was added, and the mixture was extracted with EtOAc, washed with brine, dried over $\mathrm{MgSO}_{4}$, and the organic extracts concentrated in vacuo. The residue was purified by silica gel flash chromatography using petroleum ether/EtOAc (20:1 to 5:1) to afford the product $3 f, 249.6 \mathrm{mg}, 82 \%$ yield.

\section{Characterization of Products}

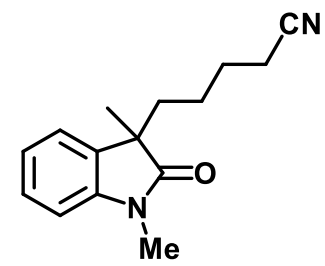

5-(1,3-dimethyl-2-oxoindolin-3-yl)pentanenitrile (3a). ${ }^{3}$ Following the General procedure $A$, the product was purified by flash chromatography on silica gel (petroleum ether/EtOAc, 20:1 to $5: 1), 35.2 \mathrm{mg}$, yield: $58 \%$, colorless oil. ${ }^{1} \mathrm{H}$ NMR $\left(\mathrm{CDCl}_{3}\right.$, $300 \mathrm{MHz}$ ): $\delta 7.26-7.21(\mathrm{~m}, 1 \mathrm{H}), 7.13(\mathrm{~d}, J=7.1 \mathrm{~Hz}, 1 \mathrm{H}), 7.03(\mathrm{t}, J=7.4 \mathrm{~Hz}, 1 \mathrm{H}), 6.82(\mathrm{~d}, J=7.7$ $\mathrm{Hz}, 1 \mathrm{H}), 3.17(\mathrm{~s}, 3 \mathrm{H}), 2.20-2.15(\mathrm{~m}, 2 \mathrm{H}), 1.93-1.83(\mathrm{~m}, 1 \mathrm{H}), 1.77-1.67(\mathrm{~m}, 1 \mathrm{H}), 1.58-1.41(\mathrm{~m}$, $2 \mathrm{H}), 1.31(\mathrm{~s}, 3 \mathrm{H}), 1.12-0.92(\mathrm{~m}, 2 \mathrm{H}) ;{ }^{13} \mathrm{C}\left\{{ }^{1} \mathrm{H}\right\} \mathrm{NMR}\left(\mathrm{CDCl}_{3}, 75 \mathrm{MHz}\right): \delta 180.3,143.2,133.5$, $127.9,122.7,122.4,119.4,108.1,48.1,37.4,26.2,25.4,23.9,23.8,16.8$.<smiles>CCN1C(=O)C(C)(CCCCC#N)c2ccccc21</smiles>

5-(1-ethyl-3-methyl-2-oxoindolin-3-yl)pentanenitrile (3b). Following the General procedure $A$, the product was purified by flash chromatography on silica gel (petroleum ether/EtOAc, 30:1 to 5:1), $42.9 \mathrm{mg}$, yield: 67\%, yellow oil. ${ }^{1} \mathrm{H} \mathrm{NMR}\left(\mathrm{CDCl}_{3}, 300\right.$ $\mathrm{MHz}): \delta 7.27-7.22(\mathrm{~m}, 1 \mathrm{H}), 7.15(\mathrm{~d}, J=7.0 \mathrm{~Hz}, 1 \mathrm{H}), 7.04(\mathrm{t}, J=7.4 \mathrm{~Hz}, 1 \mathrm{H}), 6.85(\mathrm{~d}, J=7.8 \mathrm{~Hz}$, $1 \mathrm{H}), 3.85-3.64(\mathrm{~m}, 2 \mathrm{H}), 2.19(\mathrm{t}, J=7.2 \mathrm{~Hz}, 2 \mathrm{H}), 1.96-1.86(\mathrm{~m}, 1 \mathrm{H}), 1.78-1.68(\mathrm{~m}, 1 \mathrm{H}), 1.57-1.45$ $(\mathrm{m}, 2 \mathrm{H}), 1.32(\mathrm{~s}, 3 \mathrm{H}), 1.23(\mathrm{t}, J=7.2 \mathrm{~Hz}, 3 \mathrm{H}), 1.14-0.94(\mathrm{~m}, 2 \mathrm{H}) ;{ }^{13} \mathrm{C}\left\{{ }^{1} \mathrm{H}\right\} \mathrm{NMR}\left(\mathrm{CDCl}_{3}, 75 \mathrm{MHz}\right)$ : $\delta$ 180.0, 142.3, 133.8, 127.9, 122.6, 122.5, 119.4, 108.3, 48.0, 37.5, 34.6, 25.4, 23.9, 23.7, 16.9, 12.8. IR (ATR): 2976, 2935, 2246, 1703, 1611, 1489, 1375, $756 \mathrm{~cm}^{-1}$. HRMS (ESI) m/z: $[\mathrm{M}+\mathrm{H}]^{+}$ Calcd for $\mathrm{C}_{16} \mathrm{H}_{21} \mathrm{~N}_{2} \mathrm{O}$ 257.1648; Found 257.1650. 


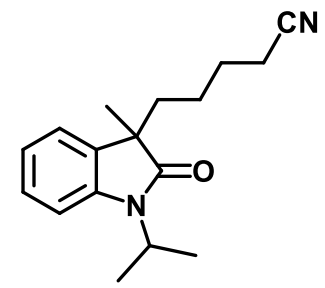

5-(1-isopropyl-3-methyl-2-oxoindolin-3-yl)pentanenitrile (3c). Following General procedure $A$, the product was purified by flash chromatography on silica gel (petroleum ether/EtOAc, $25: 1$ to $5: 1), 27.8 \mathrm{mg}$, yield: $41 \%$, colorless oil. ${ }^{1} \mathrm{H} \mathrm{NMR}\left(\mathrm{CDCl}_{3}\right.$, $300 \mathrm{MHz}): \delta$ 7.26-7.20 (m, $1 \mathrm{H}), 7.15(\mathrm{~d}, J=6.8 \mathrm{~Hz}, 1 \mathrm{H}), 7.05-7.00(\mathrm{~m}, 2 \mathrm{H}), 4.68-4.58(\mathrm{~m}, 1 \mathrm{H})$, $2.19(\mathrm{t}, J=7.2 \mathrm{~Hz}, 2 \mathrm{H}), 1.95-1.85(\mathrm{~m}, 1 \mathrm{H}), 1.77-1.67(\mathrm{~m}, 1 \mathrm{H}), 1.57-1.45(\mathrm{~m}, 8 \mathrm{H}), 1.32(\mathrm{~s}, 3 \mathrm{H})$, 1.13-0.90 (m, 2H); ${ }^{13} \mathrm{C}\left\{{ }^{1} \mathrm{H}\right\}$ NMR $\left(\mathrm{CDCl}_{3}, 75 \mathrm{MHz}\right): \delta 180.0,141.8,134.0,127.6,122.7,122.1$, $119.4,109.9,47.7,43.6,37.6,25.3,24.0,23.6,19.5,19.4,16.8$. IR (ATR): 2973, 2934, 2869, 2246, 1770, 1701, 1609, 1484, $755 \mathrm{~cm}^{-1}$. HRMS (ESI) m/z: $[\mathrm{M}+\mathrm{H}]^{+}$Calcd for $\mathrm{C}_{17} \mathrm{H}_{23} \mathrm{~N}_{2} \mathrm{O}$ 271.1805; Found 271.1806.<smiles>CCCCN1C(=O)C(C)(CCCCC#N)c2ccccc21</smiles>

5-(1-butyl-3-methyl-2-oxoindolin-3-yl)pentanenitrile (3d). Following the General procedure $A$, the product was purified by flash chromatography on silica gel (petroleum ether/EtOAc, $25: 1$ to $5: 1), 30.4 \mathrm{mg}$, yield: $43 \%$, colorless oil. ${ }^{1} \mathrm{H} \mathrm{NMR}\left(\mathrm{CDCl}_{3}\right.$, $300 \mathrm{MHz}): \delta$ 7.27-7.22 (m, 1H), $7.15(\mathrm{~d}, J=6.9 \mathrm{~Hz}, 1 \mathrm{H}), 7.06-7.02(\mathrm{~m}, 1 \mathrm{H}), 6.85(\mathrm{~d}, J=7.7 \mathrm{~Hz}$, $1 \mathrm{H}), 3.78-3.61(\mathrm{~m}, 2 \mathrm{H}), 2.22-2.17(\mathrm{~m}, 2 \mathrm{H}), 1.96-1.86(\mathrm{~m}, 1 \mathrm{H}), 1.79-1.70(\mathrm{~m}, 1 \mathrm{H}), 1.69-1.46(\mathrm{~m}$, 4H), $1.40-1.33(\mathrm{~m}, 5 \mathrm{H}), 1.17-1.00(\mathrm{~m}, 2 \mathrm{H}), 0.94(\mathrm{t}, J=7.3 \mathrm{~Hz}, 3 \mathrm{H}) ;{ }^{13} \mathrm{C}\left\{{ }^{1} \mathrm{H}\right\} \mathrm{NMR}\left(\mathrm{CDCl}_{3}, 75 \mathrm{MHz}\right)$ : $\delta$ 180.6, 177.1, 140.8, 131.9, 131.1, 128.1, 125.2, 107.7, 60.0, 47.2, 46.9, 41.4, 29.5, 27.7, 26.2, 22.0, 21.0, 13.7. IR (ATR): 2960, 2932, 2869, 2246, 1704, 1610, 1488, 1359, 755, $\mathrm{cm}^{-1}$. HRMS (ESI) $\mathrm{m} / \mathrm{z}:[\mathrm{M}+\mathrm{H}]^{+}$Calcd for $\mathrm{C}_{18} \mathrm{H}_{25} \mathrm{~N}_{2} \mathrm{O}$ 285.1961; Found 285.1963 .<smiles>CC1(CCCCC#N)C(=O)N(C2CCCCC2)c2ccccc21</smiles>

5-(1-cyclohexyl-3-methyl-2-oxoindolin-3-yl)pentanenitrile (3e). Following the General procedure $A$, the product was purified by flash chromatography on silica gel (petroleum ether/EtOAc, 30:1 to 3:1), $44.5 \mathrm{mg}$, yield: $58 \%$, colorless oil. ${ }^{1} \mathrm{H} \mathrm{NMR}\left(\mathrm{CDCl}_{3}\right.$, $300 \mathrm{MHz}): \delta$ 7.24-7.19 (m, $1 \mathrm{H}), 7.14(\mathrm{~d}, J=6.7 \mathrm{~Hz}, 1 \mathrm{H}), 7.06-7.00(\mathrm{~m}, 2 \mathrm{H}), 4.20-4.12(\mathrm{~m}, 1 \mathrm{H})$, 
2.21-2.07 (m, 4H), 1.91-1.85 (m, 3H), 1.75-1.67 (m, 4H), 1.57-1.38 (m, 4H), $1.31(\mathrm{~s}, 3 \mathrm{H}), 1.26-$ $1.21(\mathrm{~m}, 1 \mathrm{H}), 1.13-0.89(\mathrm{~m}, 2 \mathrm{H}) ;{ }^{13} \mathrm{C}\left\{{ }^{1} \mathrm{H}\right\}$ NMR $\left(\mathrm{CDCl}_{3}, 75 \mathrm{MHz}\right): \delta 180.2,142.3,134.0,127.6$, 122.6, 122.0, 119.4, 110.1, 52.1, 47.7, 37.7, 29.4, 29.2, 26.0, 25.4, 24.2, 23.7, 16.9. IR (ATR): 2931, 2860, 2246, 1702, 1601, 1484, 1361, $755 \mathrm{~cm}^{-1}$. HRMS (ESI) m/z: $[\mathrm{M}+\mathrm{H}]^{+}$Calcd for $\mathrm{C}_{20} \mathrm{H}_{27} \mathrm{~N}_{2} \mathrm{O}$ 311.2118; Found 311.2119.

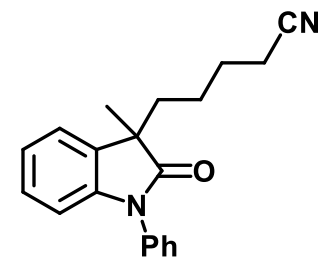

5-(3-methyl-2-oxo-1-phenylindolin-3-yl)pentanenitrile (3f). ${ }^{3}$ Following the General procedure $A$, the product was purified by flash chromatography on silica gel (petroleum ether/EtOAc, 30:1 to 5:1), $65.1 \mathrm{mg}$, yield: $86 \%$, yellow oil. ${ }^{1} \mathrm{H}$ NMR ( $\mathrm{CDCl}_{3}, 300$ $\mathrm{MHz})$ : $\delta$ 7.55-7.50 (m, 2H), 7.42-7.40 (m, 3H), 7.26-7.19 (m, 2H), 7.14-7.09 (m, $1 \mathrm{H}), 6.85(\mathrm{~d}, J$ $=7.7 \mathrm{~Hz}, 1 \mathrm{H}), 2.26-2.22(\mathrm{~m}, 2 \mathrm{H}), 2.09-1.99(\mathrm{~m}, 1 \mathrm{H}), 1.91-1.81(\mathrm{~m}, 1 \mathrm{H}), 1.64-1.53(\mathrm{~m}, 2 \mathrm{H}), 1.49$ (s, 3H), 1.34-1.13 (m, 2H); ${ }^{13} \mathrm{C}\left\{{ }^{1} \mathrm{H}\right\}$ NMR $\left(\mathrm{CDCl}_{3}, 75 \mathrm{MHz}\right): \delta 179.8,143.1,134.5,133.3,129.6$, $128.0,127.9,126.5,123.2,122.8,119.4,109.5,48.2,37.9,25.4,24.2,23.8,16.9$.<smiles>Cc1ccc(N2C(=O)C(C)(CCCCC#N)c3cc(C)ccc32)cc1</smiles>

5-(3,5-dimethyl-2-oxo-1-(p-tolyl)indolin-3-yl)pentanenitrile (3g). Following the General procedure $A$, the product was purified by flash chromatography on silica gel (petroleum ether/EtOAc, 20:1 to $5: 1), 61.9 \mathrm{mg}$, yield: $75 \%$, white solid. ${ }^{1} \mathrm{H} \mathrm{NMR}\left(\mathrm{CDCl}_{3}\right.$, $300 \mathrm{MHz}): \delta$ 7.33-7.25 (m, 4H), 7.04-6.98 (m, 2H), $6.72(\mathrm{~d}, J=8.0 \mathrm{~Hz}, 1 \mathrm{H}), 2.41(\mathrm{~s}, 3 \mathrm{H}), 2.36(\mathrm{~s}$, $3 \mathrm{H}), 2.27-2.21(\mathrm{~m}, 2 \mathrm{H}), 2.05-1.97(\mathrm{~m}, 1 \mathrm{H}), 1.88-1.78(\mathrm{~m}, 1 \mathrm{H}), 1.64-1.53(\mathrm{~m}, 2 \mathrm{H}), 1.46(\mathrm{~s}, 3 \mathrm{H})$, 1.29-1.13 (m, 2H); ${ }^{13} \mathrm{C}\left\{{ }^{1} \mathrm{H}\right\}$ NMR $\left(\mathrm{CDCl}_{3}, 75 \mathrm{MHz}\right): \delta 179.8,141.0,137.8,133.3,132.6,132.0$, $130.2,128.1,126.2,123.5,119.5,109.2,48.2,37.9,25.4,24.3,23.8,21.2,21.1,16.9$. IR (ATR): 2928, 2866, 2246, 1713, 1618, 1560, 1489, 1361, $811 \mathrm{~cm}^{-1}$. HRMS (ESI) m/z: [M+H] Calcd for $\mathrm{C}_{22} \mathrm{H}_{25} \mathrm{~N}_{2} \mathrm{O} 333.1961 ;$ Found 333.1955. 
<smiles>CC1(CCCCC#N)C(=O)N([N+](=O)[O-])c2c(Cl)cccc21</smiles>

5-(7-chloro-1,3-dimethyl-2-oxoindolin-3-yl)pentanenitrile (3h). Following the General procedure $A$, the product was purified by flash chromatography on silica gel (petroleum ether/EtOAc, 20:1 to 5:1), $24.4 \mathrm{mg}$, yield: $36 \%$, colorless oil. ${ }^{1} \mathrm{H} \mathrm{NMR}\left(\mathrm{CDCl}_{3}\right.$, $300 \mathrm{MHz}$ ): $\delta 7.18$ (dd, $J=7.9,1.3 \mathrm{~Hz}, 1 \mathrm{H}), 7.05-6.94(\mathrm{~m}, 2 \mathrm{H}), 3.57(\mathrm{~s}, 3 \mathrm{H}), 2.25-2.19(\mathrm{~m}, 2 \mathrm{H})$, 1.97-1.87 (m, 1H), 1.77-1.67 (m, 1H), 1.59-1.47 (m, 2H), $1.33(\mathrm{~s}, 3 \mathrm{H}), 1.13-0.95(\mathrm{~m}, 2 \mathrm{H}) ;{ }^{13} \mathrm{C}\left\{{ }^{1} \mathrm{H}\right\}$ $\mathrm{NMR}\left(\mathrm{CDCl}_{3}, 75 \mathrm{MHz}\right): \delta 180.6,139.2,136.4,130.4,123.6,121.0,119.5,115.7,48.1,37.7,29.6$, 25.5, 24.4, 23.8, 16.9. IR (ATR): 2936, 2868, 2246, 1713, 1607, 1463, 1367, $740 \mathrm{~cm}^{-1}$. HRMS (ESI) $\mathrm{m} / \mathrm{z}:[\mathrm{M}+\mathrm{H}]^{+}$Calcd for $\mathrm{C}_{15} \mathrm{H}_{18} \mathrm{ClN}_{2} \mathrm{O} 277.1102$; Found 277.1104 .

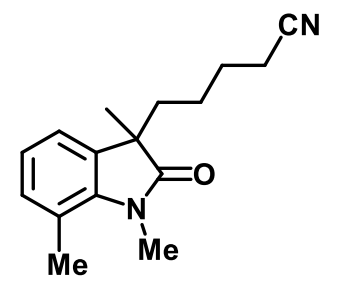

5-(1,3,7-trimethyl-2-oxoindolin-3-yl)pentanenitrile (3i). ${ }^{3}$ Following the General procedure $A$, the product was purified by flash chromatography on silica gel (petroleum ether/EtOAc, 20:1 to 3:1), $38.9 \mathrm{mg}$, yield: $61 \%$, yellow oil. ${ }^{1} \mathrm{H} \mathrm{NMR}\left(\mathrm{CDCl}_{3}, 300\right.$ $\mathrm{MHz})$ : $\delta 7.00-6.91(\mathrm{~m}, 3 \mathrm{H}), 3.48(\mathrm{~s}, 3 \mathrm{H}), 2.57(\mathrm{~s}, 3 \mathrm{H}), 2.20(\mathrm{t}, \mathrm{J}=7.1 \mathrm{~Hz}, 2 \mathrm{H}), 1.95-1.85(\mathrm{~m}, 1 \mathrm{H})$, 1.75-1.65 (m, 1H), 1.58-1.45 (m, 2H), $1.31(\mathrm{~s}, 3 \mathrm{H}), 1.10-0.95(\mathrm{~m}, 2 \mathrm{H}) ;{ }^{13} \mathrm{C}\left\{{ }^{1} \mathrm{H}\right\} \mathrm{NMR}\left(\mathrm{CDCl}_{3}, 75\right.$ MHz): $\delta 181.0,140.9,134.1,131.6,122.5,120.3,119.7,119.5,47.4,37.6,29.4,25.4,24.3$, 23.7, 19.0, 16.8 .<smiles>CN1C(=O)C(C)(CCCCC#N)c2cccc(-c3ccccc3)c21</smiles>

5-(1,3-dimethyl-2-oxo-7-phenylindolin-3-yl)pentanenitrile (3j). ${ }^{3}$ Following the General procedure $A$, the product was purified by flash chromatography on silica gel (petroleum ether/EtOAc, 20:1 to $10: 1), 50.3 \mathrm{mg}$, yield: $63 \%$, yellow oil. ${ }^{1} \mathrm{H} \mathrm{NMR}\left(\mathrm{CDCl}_{3}\right.$, $300 \mathrm{MHz}): \delta$ 7.40-7.34 (m, 5H), 7.17-7.14 (m, 1H), 7.10-7.04 (m, 2H), $2.72(\mathrm{~s}, 3 \mathrm{H}), 2.24(\mathrm{t}, J=$ $7.0 \mathrm{~Hz}, 2 \mathrm{H}), 2.00-1.90(\mathrm{~m}, 1 \mathrm{H}), 1.83-1.73(\mathrm{~m}, 1 \mathrm{H}), 1.62-1.50(\mathrm{~m}, 2 \mathrm{H}), 1.39(\mathrm{~s}, 3 \mathrm{H}), 1.18-1.06(\mathrm{~m}$, $2 \mathrm{H}) ;{ }^{13} \mathrm{C}\left\{{ }^{1} \mathrm{H}\right\}$ NMR $\left(\mathrm{CDCl}_{3}, 75 \mathrm{MHz}\right): \delta 181.4,140.1,138.9,134.6,131.0,129.9,127.8,127.7$, $125.6,122.0,121.4,119.5,47.4,37.8,30.2,25.5,24.3,23.8,16.9$. 
<smiles>Cc1ccc2c(c1)N(C)C(=O)C2(C)CCCCC#N</smiles>

$3 k$<smiles>Cc1cccc2c1C(C)(CCCCC#N)C(=O)N2C</smiles>

$3 \mathbf{k}^{\prime}$

5-(1,3,6-trimethyl-2-oxoindolin-3-yl)pentanenitrile (3k) and 5-(1,3,4-trimethyl-2oxoindolin-3-yl)pentanenitrile (3k'). ${ }^{3}$ Following the General procedure $A$, the product was purified by flash chromatography on silica gel (petroleum ether/EtOAc, 30:1 to $5: 1$ ), isolated as an inseparable mixture, $\mathbf{3 k}: \mathbf{3 \mathbf { k } ^ { \prime }}=1: 1.7,40.6 \mathrm{mg}$, total yield: $64 \%$, colorless oil. ${ }^{1} \mathrm{H}$ NMR $\left(\mathrm{CDCl}_{3}, 300 \mathrm{MHz}\right): \delta$ 7.19-7.13 $(\mathrm{m}, 0.6 \mathrm{H}), 7.03(\mathrm{~d}, J=7.5 \mathrm{~Hz}, 0.4 \mathrm{H}), 6.88-6.81(\mathrm{~m}$, $1 \mathrm{H}), 6.68(\mathrm{~d}, J=8.2 \mathrm{~Hz}, 1 \mathrm{H}), 3.18(\mathrm{~d}, J=1.8 \mathrm{~Hz}, 3 \mathrm{H}), 2.36(\mathrm{~d}, J=7.4 \mathrm{~Hz}, 3 \mathrm{H}), 2.22-2.17(\mathrm{~m}, 2 \mathrm{H})$, 2.01-1.96 (m, 1.3H), 1.93-1.83 (m, 0.5H), 1.77-1.67 (m, 0.5H), 1.58-1.46 (m, 2H), $1.41(\mathrm{~s}, 2 \mathrm{H})$, $1.31(\mathrm{~s}, 1 \mathrm{H}), 1.11-0.86(\mathrm{~m}, 2 \mathrm{H}) ;{ }^{13} \mathrm{C}\left\{{ }^{1} \mathrm{H}\right\} \mathrm{NMR}\left(\mathrm{CDCl}_{3}, 75 \mathrm{MHz}\right): \delta 180.7,180.4,143.5,143.3$, 138.0, 134.1, 130.6, 130.0, 127.8, 125.2, 123.2, 122.2, 119.5, 119.5, 109.1, 106.0, 49.3, 48.0, $37.5,35.4,26.3,26.2,25.5,25.4,24.2,24.0,23.9,22.4,21.8,18.2,16.9,16.8$.<smiles>Cc1ccc2c(c1)C(C)(CCCCC#N)C(=O)N2C</smiles>

5-(1,3,5-trimethyl-2-oxoindolin-3-yl)pentanenitrile (3I). ${ }^{3}$ Following the General procedure $A$, the product was purified by flash chromatography on silica gel (petroleum ether/EtOAc, 20:1 to 5:1), $41.9 \mathrm{mg}$, yield: $66 \%$, colorless oil. ${ }^{1} \mathrm{H} \mathrm{NMR}\left(\mathrm{CDCl}_{3}\right.$, $300 \mathrm{MHz}$ ): $\delta 7.05$ (d, J= $7.8 \mathrm{~Hz}, 1 \mathrm{H}), 6.96(\mathrm{~s}, 1 \mathrm{H}), 6.72(\mathrm{~d}, J=7.9 \mathrm{~Hz}, 1 \mathrm{H}), 3.18(\mathrm{~s}, 3 \mathrm{H}), 2.33(\mathrm{~s}$, $3 \mathrm{H}), 2.23-2.17(\mathrm{~m}, 2 \mathrm{H}), 1.94-1.84(\mathrm{~m}, 1 \mathrm{H}), 1.77-1.67(\mathrm{~m}, 1 \mathrm{H}), 1.58-1.46(\mathrm{~m}, 2 \mathrm{H}), 1.32(\mathrm{~s}, 3 \mathrm{H})$, 1.11-0.97 (m, 2H); ${ }^{13} \mathrm{C}\left\{{ }^{1} \mathrm{H}\right\}$ NMR $\left(\mathrm{CDCl}_{3}, 75 \mathrm{MHz}\right): \delta 180.3,140.8,133.6,132.2,128.2,123.3$, $119.5,107.9,48.2,37.5,26.2,25.5,24.0,23.8,21.2,16.9$.<smiles>COc1ccc2c(c1)C(C)(CCCCC#N)C(=O)N2C</smiles>

5-(5-methoxy-1,3-dimethyl-2-oxoindolin-3-yl)pentanenitrile $(3 \mathrm{~m}) .^{3}$ Following the General procedure $A$, the product was purified by flash chromatography on silica gel (petroleum ether/EtOAc, 15:1 to 5:1), $36.8 \mathrm{mg}$, yield: $54 \%$, yellow oil. ${ }^{1} \mathrm{H}$ NMR $\left(\mathrm{CDCl}_{3}, 300\right.$ $\mathrm{MHz})$ : $\delta$ 6.79-6.71 (m, 3H), 3.78 (s, 3H), 3.17 (s, 3H), 2.22-2.17 (m, 2H), 1.94-1.85 (m, 1H), 1.75$1.66(\mathrm{~m}, 1 \mathrm{H}), 1.57-1.45(\mathrm{~m}, 2 \mathrm{H}), 1.32(\mathrm{~s}, 3 \mathrm{H}), 1.13-0.93(\mathrm{~m}, 2 \mathrm{H}) ;{ }^{13} \mathrm{C}\left\{{ }^{1} \mathrm{H}\right\} \mathrm{NMR}\left(\mathrm{CDCl}_{3}, 75 \mathrm{MHz}\right)$ : $\delta 180.0,156.2,136.8,135.0,119.5,111.8,110.3,108.4,55.8,48.6,37.5,26.3,25.5,24.0,23.8$, 
<smiles>CN1C(=O)C(C)(CCCCC#N)c2cc(F)ccc21</smiles>

5-(5-fluoro-1,3-dimethyl-2-oxoindolin-3-yl)pentanenitrile (3n). Following the General procedure $A$, the product was purified by flash chromatography on silica gel (petroleum ether/EtOAc, 15:1 to 5:1), $29.1 \mathrm{mg}$, yield: $45 \%$, colorless oil. ${ }^{1} \mathrm{H} \mathrm{NMR}\left(\mathrm{CDCl}_{3}\right.$, $300 \mathrm{MHz}): \delta 6.98-6.87(\mathrm{~m}, 2 \mathrm{H}), 6.78-6.73(\mathrm{~m}, 1 \mathrm{H}), 3.18(\mathrm{~s}, 3 \mathrm{H}), 2.20(\mathrm{t}, J=7.2 \mathrm{~Hz}, 2 \mathrm{H}), 1.95-$ $1.85(\mathrm{~m}, 1 \mathrm{H}), 1.76-1.66(\mathrm{~m}, 1 \mathrm{H}), 1.58-1.44(\mathrm{~m}, 2 \mathrm{H}), 1.32(\mathrm{~s}, 3 \mathrm{H}), 1.13-0.93(\mathrm{~m}, 2 \mathrm{H}) ;{ }^{19} \mathrm{~F} \mathrm{NMR}$ ( $\left.\mathrm{CDCl}_{3}, 282 \mathrm{MHz}\right): \delta-120.4(\mathrm{~s}, 1 \mathrm{~F}) ;{ }^{13} \mathrm{C}\left\{{ }^{1} \mathrm{H}\right\} \mathrm{NMR}\left(\mathrm{CDCl}_{3}, 75 \mathrm{MHz}\right): \delta 180.0,159.5(J=239.2 \mathrm{~Hz})$, $139.1(J=1.8 \mathrm{~Hz}), 135.3(J=7.7 \mathrm{~Hz}), 119.4,114.4(J=23.3 \mathrm{~Hz}), 110.7(J=24.4 \mathrm{~Hz}), 108.7(J=$ $8.1 \mathrm{~Hz}$ ), 48.7, 37.4, 26.4, 25.4, 23.8, 23.8, 16.9 . IR (ATR): 2935, 2868, 2246, 1706, 1621, 1493, $1351,741 \mathrm{~cm}^{-1}$. HRMS (ESI) m/z: [M+Na] ${ }^{+}$Calcd for $\mathrm{C}_{15} \mathrm{H}_{17} \mathrm{FN}_{2} \mathrm{NaO} 283.1217$; Found 283.1226.<smiles>CC1(CCCCC#N)C(=O)N([N+](=O)[O-])c2ccc(C(F)(F)F)cc21</smiles>

5-(1,3-dimethyl-2-oxo-5-(trifluoromethyl)indolin-3-yl)pentanenitrile (3o). ${ }^{3}$ Following the General procedure $A$, the product was purified by flash chromatography on silica gel (petroleum ether/EtOAc, 15:1 to 3:1), $58.2 \mathrm{mg}$, yield: 75\%, yellow oil. ${ }^{1} \mathrm{H}$ NMR $\left(\mathrm{CDCl}_{3}\right.$, $300 \mathrm{MHz}$ ): $\delta 7.55$ (d, J = $8.1 \mathrm{~Hz}, 1 \mathrm{H}), 7.37(\mathrm{~s}, 1 \mathrm{H}), 6.92(\mathrm{~d}, J=8.2 \mathrm{~Hz}, 1 \mathrm{H}), 3.23(\mathrm{~s}, 3 \mathrm{H}), 2.21(\mathrm{t}, J$ $=7.2 \mathrm{~Hz}, 2 \mathrm{H}), 1.99-1.89(\mathrm{~m}, 1 \mathrm{H}), 1.82-1.72(\mathrm{~m}, 1 \mathrm{H}), 1.59-1.46(\mathrm{~m}, 2 \mathrm{H}), 1.36(\mathrm{~s}, 3 \mathrm{H}), 1.14-0.93$ $(\mathrm{m}, 2 \mathrm{H}) ;{ }^{19} \mathrm{~F} \mathrm{NMR}\left(\mathrm{CDCl}_{3}, 282 \mathrm{MHz}\right): \delta-61.3(\mathrm{~s}, 3 \mathrm{~F}) ;{ }^{13} \mathrm{C}\left\{{ }^{1} \mathrm{H}\right\} \mathrm{NMR}\left(\mathrm{CDCl}_{3}, 75 \mathrm{MHz}\right): \delta 180.3$, $146.3,134.2,125.9$ (q, $J=4.0 \mathrm{~Hz}$ ), 124.9 (q, $J=32.3 \mathrm{~Hz}$ ), 122.7, 119.5 (q, $J=3.6 \mathrm{~Hz}$ ), 119.4, $108.0,48.2,37.4,26.4,25.4,23.8,23.7,16.9$.<smiles>CN1C(=O)C(C)(CCCCC#N)c2cc([N+](=O)[O-])ccc21</smiles>

5-(1,3-dimethyl-5-nitro-2-oxoindolin-3-yl)pentanenitrile (3p). ${ }^{3}$ Following the General procedure $A$, the product was purified by flash chromatography on silica gel (petroleum ether/EtOAc, 10:1 to 3:1), $23 \mathrm{mg}$, yield: 32\%, yellow oil. ${ }^{1} \mathrm{H}$ NMR $\left(\mathrm{CDCl}_{3}, 300\right.$ $\mathrm{MHz}): \delta 8.25$ (dd, J = 8.6, 2.2 Hz, 1H), $8.04(\mathrm{~d}, J=2.1 \mathrm{~Hz}, 1 \mathrm{H}), 6.94(\mathrm{~d}, J=8.6 \mathrm{~Hz}, 1 \mathrm{H}), 3.27(\mathrm{~s}$, $3 \mathrm{H}), 2.23(\mathrm{t}, J=7.1 \mathrm{~Hz}, 2 \mathrm{H}), 2.02-1.92(\mathrm{~m}, 1 \mathrm{H}), 1.86-1.76(\mathrm{~m}, 1 \mathrm{H}), 1.60-1.49(\mathrm{~m}, 2 \mathrm{H}), 1.40(\mathrm{~s}$, 
3H), 1.13-0.93 (m, 2H); ${ }^{13} \mathrm{C}\left\{{ }^{1} \mathrm{H}\right\} \mathrm{NMR}\left(\mathrm{CDCl}_{3}, 75 \mathrm{MHz}\right): \delta 180.4,149.0,143.6,134.4,125.5,119.3$, $118.4,107.8,48.3,37.3,26.7,25.3,23.7,23.7,16.9$.

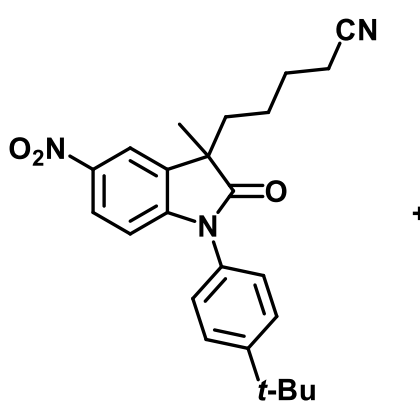

$3 q$<smiles>CC(C)(C)c1ccc2c(c1)C(C)(CCCCC#N)C(=O)N2c1ccc([N+](=O)[O-])cc1</smiles>

$3 \mathbf{q}^{\prime}$

5-(1-(4-(tert-butyl)phenyl)-3-methyl-5-nitro-2-oxoindolin-3-yl)pentanenitrile (3q) and 5-(5-(tert-butyl)-3-methyl-1-(4-nitrophenyl)-2-oxoindolin-3-yl)pentanenitrile $\left(\mathbf{3} \mathbf{q}^{\prime}\right)$. Following the General procedure $A$, the product was purified by flash chromatography on silica gel (petroleum ether/EtOAc, 20:1 to 5:1), isolated as an inseparable mixture, $\mathbf{3 q}: \mathbf{3 \mathbf { q } ^ { \prime }}=1.2: 1,46.5 \mathrm{mg}$, total yield: $46 \%$, yellow oil. ${ }^{1} \mathrm{H} \mathrm{NMR}\left(\mathrm{CDCl}_{3}\right.$, $300 \mathrm{MHz}): \delta 8.37(\mathrm{~d}, J=8.9 \mathrm{~Hz}, 1 \mathrm{H}), 8.18-8.13(\mathrm{~m}, 1 \mathrm{H}), 7.68(\mathrm{~d}, J=8.9 \mathrm{~Hz}, 1 \mathrm{H}), 7.56(\mathrm{~d}, J=8.5$ $\mathrm{Hz}, 1 \mathrm{H}), 7.28(\mathrm{~d}, J=8.3 \mathrm{~Hz}, 2 \mathrm{H}), 6.93(\mathrm{~d}, J=8.7 \mathrm{~Hz}, 1 \mathrm{H}), 2.29-2.24(\mathrm{~m}, 2 \mathrm{H}), 2.15-1.82(\mathrm{~m}, 2 \mathrm{H})$, 1.66-1.49 (m, 5H), $1.35(\mathrm{~d}, J=5.7 \mathrm{~Hz}, 9 \mathrm{H}), 1.28-1.22(\mathrm{~m}, 2 \mathrm{H}) ;{ }^{13} \mathrm{C}\left\{{ }^{1} \mathrm{H}\right\} \mathrm{NMR}\left(\mathrm{CDCl}_{3}, 75 \mathrm{MHz}\right): \delta$ 179.9, 179.8, 152.2, 149.0, 147.7, 146.1, 143.8, 140.6, 138.9, 134.2, 133.1, 130.6, 127.0, 126.1, 126.0, 125.3, 125.0, 124.8, 120.2, 119.4, 119.3, 118.7, 109.3, 109.1, 48.7, 48.4, 38.0, 37.8, 34.9, 34.8, 31.6, 31.3, 25.3, 24.4, 24.0, 23.8, 23.8, 17.0, 16.9. IR (ATR): 2960, 2869, 2246, 1729, 1614, 1517, 1336, $755 \mathrm{~cm}^{-1}$. HRMS (ESI) m/z: $[\mathrm{M}+\mathrm{Na}]^{+}$Calcd for $\mathrm{C}_{24} \mathrm{H}_{27} \mathrm{~N}_{3} \mathrm{NaO}_{3}$ 428.1945; Found 428.1952.<smiles>Cn1c(=O)c(CCCCC#N)c([PH2+])c2ccccc21</smiles>

5-(1-methyl-2-oxo-3-phenylindolin-3-yl)pentanenitrile (3r). Following the General procedure $A$, the product was purified by flash chromatography on silica gel (petroleum ether/EtOAc, 20:1 to 3:1), $23.5 \mathrm{mg}$, yield: $31 \%$, colorless oil. ${ }^{1} \mathrm{H} \mathrm{NMR}\left(\mathrm{CDCl}_{3}\right.$, $300 \mathrm{MHz}): \delta$ 7.37-7.23 (m, 7H), 7.17-7.12 (m, 1H), $6.93(\mathrm{~d}, J=7.8 \mathrm{~Hz}, 1 \mathrm{H}), 3.23(\mathrm{~s}, 3 \mathrm{H}), 2.45-$ $2.35(\mathrm{~m}, 1 \mathrm{H}), 2.26-2.16(\mathrm{~m}, 3 \mathrm{H}), 1.68-1.55(\mathrm{~m}, 2 \mathrm{H}), 1.31-1.03(\mathrm{~m}, 2 \mathrm{H}) ;{ }^{13} \mathrm{C}\left\{{ }^{1} \mathrm{H}\right\} \mathrm{NMR}\left(\mathrm{CDCl}_{3}, 75\right.$ $\mathrm{MHz}): \delta 178.4,143.9,139.9,131.7,128.7,128.5,127.5,126.8,124.8,122.9,119.5,108.6,56.4$, 37.1, 26.5, 25.6, 24.0, 17.0. IR (ATR): 2937, 2867, 2246, 1706, 1611, 1493, 1371, $754 \mathrm{~cm}^{-1}$. HRMS (ESI) m/z: [M+Na] ${ }^{+}$Calcd for $\mathrm{C}_{20} \mathrm{H}_{20} \mathrm{~N}_{2} \mathrm{NaO}$ 327.1468; Found 327.1469. 
<smiles>CN1C(=O)C(C)(CCC(CC#N)c2ccccc2)c2ccccc21</smiles>

5-(1,3-dimethyl-2-oxoindolin-3-yl)-3-phenylpentanenitrile (3s). ${ }^{3}$ Following the General procedure $A$, the product was purified by flash chromatography on silica gel (petroleum ether/EtOAc, 25:1 to 5:1), Isolated as an inseparable mixture, $d r=1: 1,43.2$ $\mathrm{mg}$, yield: $54 \%$, colorless oil. ${ }^{1} \mathrm{H}$ NMR $\left(\mathrm{CDCl}_{3}, 300 \mathrm{MHz}\right): \delta$ 7.34-7.25 $(\mathrm{m}, 4 \mathrm{H}), 7.16-7.03(\mathrm{~m}$, $3.5 \mathrm{H}), 6.96(\mathrm{~d}, J=7.3 \mathrm{~Hz}, 0.5 \mathrm{H}), 6.86(\mathrm{~d}, J=7.7 \mathrm{~Hz}, 1 \mathrm{H}), 3.22(\mathrm{~d}, J=4.8 \mathrm{~Hz}, 3 \mathrm{H}), 2.86-2.69(\mathrm{~m}$, 1H), 2.46-2.41 (m, 2H), 1.90-1.57 (m, 2H), 1.54-1.36 (m, $2 \mathrm{H}), 1.28(\mathrm{~d}, J=8.3 \mathrm{~Hz}, 3 \mathrm{H}) ;{ }^{13} \mathrm{C}\left\{{ }^{1} \mathrm{H}\right\}$ NMR ( $\left.\mathrm{CDCl}_{3}, 75 \mathrm{MHz}\right): \delta 180.2,180.1,143.2,143.1,140.9,140.8,133.5,133.3,128.9,128.0$, $127.5,127.5,127.2,127.1,122.7,122.6,122.4,122.3,118.2,108.2,48.0,48.0,42.2,42.1,35.7$, $35.6,29.4,26.2,25.4,25.0,23.9,23.8$.

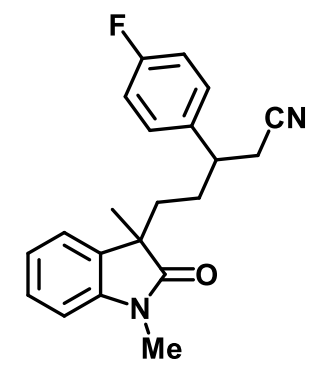

5-(1,3-dimethyl-2-oxoindolin-3-yl)-3-(4-fluorophenyl)pentanenitrile (3t). Following the General procedure $A$, the product was purified by flash chromatography on silica gel (petroleum ether/EtOAc, 25:1 to $5: 1$ ), Isolated as an inseparable mixture, $d r=1: 1.2$, $44.8 \mathrm{mg}$, yield: $53 \%$, colorless oil. ${ }^{1} \mathrm{H}$ NMR $\left(\mathrm{CDCl}_{3}, 300 \mathrm{MHz}\right): \delta 7.31-7.26(\mathrm{~m}, 1 \mathrm{H}), 7.15-6.94$ $(\mathrm{m}, 6 \mathrm{H}), 6.86(\mathrm{~d}, J=7.7 \mathrm{~Hz}, 1 \mathrm{H}), 3.21(\mathrm{~d}, J=3.8 \mathrm{~Hz}, 3 \mathrm{H}), 2.85-2.69(\mathrm{~m}, 1 \mathrm{H}), 2.49-2.33(\mathrm{~m}, 2 \mathrm{H})$, 1.83-1.63 (m, 1H), 1.54-1.33 (m, 2H), 1.28-1.14 (m, 4H); $\left.{ }^{19} \mathrm{~F} \mathrm{NMR} \mathrm{(CDCl}, 282 \mathrm{MHz}\right): \delta-114.8(\mathrm{~s}$, 1F), -114.9 (s, 1F); ${ }^{13} \mathrm{C}\left\{{ }^{1} \mathrm{H}\right\}$ NMR (CDCl $3,75 \mathrm{MHz}$ ): $\delta 180.2,180.1,162.0$ (d, $J=244.4 \mathrm{~Hz}$ ), 162.0 (d, $J=244.6 \mathrm{~Hz}), 143.2,143.2,136.6(\mathrm{~d}, J=8.1 \mathrm{~Hz}), 136.5(\mathrm{~d}, J=8.0 \mathrm{~Hz}), 133.4,133.2,128.8(\mathrm{~d}$, $J=7.9 \mathrm{~Hz}$ ), $128.7(\mathrm{~d}, J=8.0 \mathrm{~Hz}), 128.1,122.7,122.7,122.4,122.3,118.0,115.8(\mathrm{~d}, J=21.3 \mathrm{~Hz}$ ), 115.8 (d, $J=21.2 \mathrm{~Hz}$ ), 108.2, 48.0, 48.0, 41.5, 41.5, 35.7, 35.6, 29.6, 29.5, 26.2, 25.5, 25.2, 23.9, 23.9. IR (ATR): 2929, 2248, 1705, 1612, 1510, 1375, 1350, $753 \mathrm{~cm}^{-1}$. HRMS (ESI) m/z: [M+Na] Calcd for $\mathrm{C}_{21} \mathrm{H}_{21} \mathrm{FN}_{2} \mathrm{NaO}$ 359.1530; Found 359.1533. 
<smiles>Cc1ccc(C(CCN)CCC2(C)C(=O)N(C)c3ccccc32)cc1</smiles>

5-(1,3-dimethyl-2-oxoindolin-3-yl)-3-(p-tolyl)pentanenitrile (3u). ${ }^{3}$ Following the General procedure $A$, the product was purified by flash chromatography on silica gel (petroleum ether/EtOAc, 20:1 to 5:1), Isolated as an inseparable mixture, $d r=1: 1.1,44.2$ $\mathrm{mg}$, yield: $53 \%$, colorless oil. ${ }^{1} \mathrm{H} \mathrm{NMR}\left(\mathrm{CDCl}_{3}, 300 \mathrm{MHz}\right): \delta$ 7.31-7.26 $(\mathrm{m}, 1 \mathrm{H}), 7.16-6.92(\mathrm{~m}, 6 \mathrm{H})$, $6.86(\mathrm{~d}, J=7.4 \mathrm{~Hz}, 1 \mathrm{H}), 3.22(\mathrm{~d}, J=4.8 \mathrm{~Hz}, 3 \mathrm{H}), 2.83-2.66(\mathrm{~m}, 1 \mathrm{H}), 2.44-2.39(\mathrm{~m}, 2 \mathrm{H}), 2.33(\mathrm{~d}, J$ $=5.5 \mathrm{~Hz}, 3 \mathrm{H}), 1.89-1.73(\mathrm{~m}, 1 \mathrm{H}), 1.75-1.54(\mathrm{~m}, 1 \mathrm{H}), 1.51-1.34(\mathrm{~m}, 2 \mathrm{H}), 1.28(\mathrm{~d}, J=7.5 \mathrm{~Hz}, 3 \mathrm{H})$; ${ }^{13} \mathrm{C}\left\{{ }^{1} \mathrm{H}\right\}$ NMR $\left(\mathrm{CDCl}_{3}, 75 \mathrm{MHz}\right): \delta 180.3,180.2,143.2,143.2,137.9,137.8,137.1,137.1,133.6$, 133.4, 129.6, 128.0, 127.1, 127.0, 122.7, 122.6, 122.4, 122.4, 118.3, 118.3, 108.2, 48.1, 48.0, $41.8,41.8,35.8,35.7,29.5,29.4,26.2,26.0,25.2,24.0,23.8,21.1,21.1$.<smiles>CC1(CCCCC#N)C(=O)N2CCCc3cccc1c32</smiles>

5-(1-methyl-2-oxo-1,2,5,6-tetrahydro-4H-pyrrolo[3,2,1-ij]quinolin-1yl)pentanenitrile (3v). ${ }^{3}$ Following the General procedure $A$, the product was purified by flash chromatography on silica gel (petroleum ether/EtOAc, 15:1 to 3:1), $23.8 \mathrm{mg}$, yield: $35 \%$, colorless liquid. ${ }^{1} \mathrm{H}$ NMR $\left(\mathrm{CDCl}_{3}, 300 \mathrm{MHz}\right): \delta 7.01-6.90(\mathrm{~m}, 3 \mathrm{H}), 3.70(\mathrm{t}, J=5.8 \mathrm{~Hz}$, $2 \mathrm{H}), 2.78(\mathrm{t}, J=6.0 \mathrm{~Hz}, 2 \mathrm{H}), 2.21(\mathrm{t}, J=7.0 \mathrm{~Hz}, 2 \mathrm{H}), 2.02-1.94(\mathrm{~m}, 2 \mathrm{H}), 1.92-1.82(\mathrm{~m}, 1 \mathrm{H}), 1.79-$ $1.69(\mathrm{~m}, 1 \mathrm{H}), 1.58-1.47(\mathrm{~m}, 2 \mathrm{H}), 1.33(\mathrm{~s}, 3 \mathrm{H}), 1.17-1.03(\mathrm{~m}, 2 \mathrm{H}) ;{ }^{13} \mathrm{C}\left\{{ }^{1} \mathrm{H}\right\} \mathrm{NMR}\left(\mathrm{CDCl}_{3}, 75 \mathrm{MHz}\right)$ : $\delta 179.2,138.9,132.0,126.7,122.1,120.3,120.2,119.5,49.4,38.7,37.1,25.4,24.5,23.8,23.5$, $21.2,16.8$.<smiles></smiles>

5-(2,4-dimethyl-1,3-dioxo-1,2,3,4-tetrahydroisoquinolin-4-yl)pentanenitrile (4a). ${ }^{4}$ Following the General procedure $B$, the product was purified by flash chromatography on silica gel (petroleum ether/EtOAc, $20: 1$ to $10: 1$ ), $43.9 \mathrm{mg}$, yield: $65 \%$, yellow oil. ${ }^{1} \mathrm{H}$ $\operatorname{NMR}\left(\mathrm{CDCl}_{3}, 300 \mathrm{MHz}\right): \delta 8.22(\mathrm{~d}, J=7.9 \mathrm{~Hz}, 1 \mathrm{H}), 7.66-7.61(\mathrm{~m}, 1 \mathrm{H}), 7.44-7.38(\mathrm{~m}, 2 \mathrm{H}), 3.35(\mathrm{~s}$, $3 \mathrm{H}), 2.35-2.25(\mathrm{~m}, 1 \mathrm{H}), 2.20-2.15(\mathrm{~m}, 2 \mathrm{H}), 1.94-1.84(\mathrm{~m}, 1 \mathrm{H}), 1.58(\mathrm{~s}, 3 \mathrm{H}), 1.55-1.42(\mathrm{~m}, 2 \mathrm{H})$, 1.08-0.81 (m, 2H); ${ }^{13} \mathrm{C}\left\{{ }^{1} \mathrm{H}\right\} \mathrm{NMR}\left(\mathrm{CDCl}_{3}, 75 \mathrm{MHz}\right): \delta 176.4,164.2,143.0,134.2,129.0,127.6$, $125.1,124.8,119.2,47.6,41.7,29.7,27.2,25.3,24.4,16.8$. 
<smiles>Cc1ccc2c(c1)C(CCCC#N)(CCCCC#N)C(=O)N2C</smiles>

5-(2,4,6-trimethyl-1,3-dioxo-1,2,3,4-tetrahydroisoquinolin-4-yl)pentanenitrile (4b). ${ }^{4}$ Following the General procedure $B$, the product was purified by flash chromatography on silica gel (petroleum ether/EtOAc, 20:1 to $10: 1), 19.6 \mathrm{mg}$, yield: $26 \%$, colorless oil. ${ }^{1} \mathrm{H}$ $\operatorname{NMR}\left(\mathrm{CDCl}_{3}, 300 \mathrm{MHz}\right): \delta 8.12(\mathrm{~d}, J=8.0 \mathrm{~Hz}, 1 \mathrm{H}), 7.24(\mathrm{~d}, J=8.9 \mathrm{~Hz}, 1 \mathrm{H}), 7.18(\mathrm{~s}, 1 \mathrm{H}), 3.36(\mathrm{~s}$, $3 \mathrm{H}), 2.45(\mathrm{~s}, 3 \mathrm{H}), 2.35-2.17(\mathrm{~m}, 3 \mathrm{H}), 1.93-1.83(\mathrm{~m}, 1 \mathrm{H}), 1.59-1.47(\mathrm{~m}, 5 \mathrm{H}), 1.07-0.87(\mathrm{~m}, 2 \mathrm{H})$; ${ }^{13} \mathrm{C}\left\{{ }^{1} \mathrm{H}\right\} \mathrm{NMR}\left(\mathrm{CDCl}_{3}, 75 \mathrm{MHz}\right): \delta 176.7,164.4,145.3,143.1,129.1,128.8,125.5,122.5,119.3$, $47.6,41.8,29.8,27.2,25.4,24.5,22.1,16.9$.<smiles>CN1C(=O)c2ccc(Cl)cc2CC1(CCCCC#N)C(N)=O</smiles>

5-(6-chloro-2,4-dimethyl-1,3-dioxo-1,2,3,4-tetrahydroisoquinolin-4yl)pentanenitrile (4c). ${ }^{4}$ Following the General procedure $B$, the product was purified by flash chromatography on silica gel (petroleum ether/EtOAc, 20:1 to 10:1), $46 \mathrm{mg}$, yield: $60 \%$, white solid. ${ }^{1} \mathrm{H}$ NMR $\left(\mathrm{CDCl}_{3}, 300 \mathrm{MHz}\right): \delta 8.16(\mathrm{~d}, J=8.4 \mathrm{~Hz}, 1 \mathrm{H}), 7.42-7.37(\mathrm{~m}, 2 \mathrm{H})$, $3.34(\mathrm{~s}, 3 \mathrm{H}), 2.35-2.17(\mathrm{~m}, 3 \mathrm{H}), 1.89-1.79(\mathrm{~m}, 1 \mathrm{H}), 1.58-1.47(\mathrm{~m}, 5 \mathrm{H}), 1.05-0.87(\mathrm{~m}, 2 \mathrm{H}) ;{ }^{13} \mathrm{C}\left\{{ }^{1} \mathrm{H}\right\}$ NMR $\left(\mathrm{CDCl}_{3}, 75 \mathrm{MHz}\right): \delta 175.7,163.4,144.8,140.9,130.6,128.3,125.3,124.0,119.2,47.7$, $41.8,29.5,27.3,25.2,24.4,16.8$.<smiles>CN1C(=O)c2ccc(C#N)cc2C(CCCC#N)(CCCCC#N)C1=O</smiles>

4-(4-cyanobutyl)-2,4-dimethyl-1,3-dioxo-1,2,3,4-tetrahydroisoquinoline-6-

carbonitrile (4d). ${ }^{4}$ Following the General procedure $B$, the product was purified by flash chromatography on silica gel (petroleum ether/EtOAc, 15:1 to 5:1), $47.7 \mathrm{mg}$, yield: $65 \%$, white solid. ${ }^{1} \mathrm{H} N M R\left(\mathrm{CDCl}_{3}, 300 \mathrm{MHz}\right): \delta 8.35(\mathrm{~d}, J=8.6 \mathrm{~Hz}, 1 \mathrm{H}), 7.73-7.71(\mathrm{~m}, 2 \mathrm{H}), 3.38$ $(\mathrm{s}, 3 \mathrm{H}), 2.40-2.30(\mathrm{~m}, 1 \mathrm{H}), 2.22(\mathrm{t}, J=7.1 \mathrm{~Hz}, 2 \mathrm{H}), 1.93-1.83(\mathrm{~m}, 1 \mathrm{H}), 1.62-1.49(\mathrm{~m}, 5 \mathrm{H}), 1.10-$ $0.98(\mathrm{~m}, 1 \mathrm{H}), 0.94-0.79(\mathrm{~m}, 1 \mathrm{H}) ;{ }^{13} \mathrm{C}\left\{{ }^{1} \mathrm{H}\right\}$ NMR $\left(\mathrm{CDCl}_{3}, 75 \mathrm{MHz}\right): \delta 175.1,162.8,144.1,130.8$, $129.9,129.5,128.2,119.1,117.8,117.6,47.7,41.7,29.5,27.6,25.1,24.4,16.9$.<smiles>CN1C(=O)c2ccc(C(F)(F)F)cc2C(CCCC#N)(CCCC#N)C1=O</smiles>

5-(2,4-dimethyl-1,3-dioxo-6-(trifluoromethyl)-1,2,3,4-tetrahydroisoquinolin-4- 
yl)pentanenitrile (4e). ${ }^{4}$ Following the General procedure $B$, the product was purified by flash chromatography on silica gel (petroleum ether/EtOAc, 20:1 to 5:1), $51.1 \mathrm{mg}$, yield: $61 \%$, white solid. ${ }^{1} \mathrm{H} \mathrm{NMR}\left(\mathrm{CDCl}_{3}, 300 \mathrm{MHz}\right): \delta 8.36(\mathrm{~d}, J=8.2 \mathrm{~Hz}, 1 \mathrm{H}), 7.68(\mathrm{~d}, J=8.3 \mathrm{~Hz}$, $1 \mathrm{H}), 7.64(\mathrm{~s}, 1 \mathrm{H}), 3.37(\mathrm{~s}, 3 \mathrm{H}), 2.39-2.29(\mathrm{~m}, 1 \mathrm{H}), 2.20(\mathrm{t}, J=7.2 \mathrm{~Hz}, 2 \mathrm{H}), 1.95-1.85(\mathrm{~m}, 1 \mathrm{H}), 1.63$ $(\mathrm{s}, 3 \mathrm{H}), 1.59-1.47(\mathrm{~m}, 2 \mathrm{H}), 1.11-0.98(\mathrm{~m}, 1 \mathrm{H}), 0.93-0.78(\mathrm{~m}, 1 \mathrm{H}) ;{ }^{19} \mathrm{~F} \mathrm{NMR}\left(\mathrm{CDCl}_{3}, 282 \mathrm{MHz}\right): \delta$ $-63.1(\mathrm{~s}, 3 \mathrm{~F}) ;{ }^{13} \mathrm{C}\left\{{ }^{1} \mathrm{H}\right\} \mathrm{NMR}\left(\mathrm{CDCl}_{3}, 75 \mathrm{MHz}\right): \delta 175.5,163.1,143.9,135.7$ (q, $\left.J=32.6 \mathrm{~Hz}\right), 130.0$, $127.8,124.4(q, J=3.5 \mathrm{~Hz}), 123.4(q, J=271.3 \mathrm{~Hz}), 122.2(q, J=3.8 \mathrm{~Hz}), 119.1,47.8,41.8,29.3$, $27.4,25.1,24.3,16.7$.<smiles>CCC1(CCCCC#N)C(=O)N(C)C(=O)c2cc(C)ccc21</smiles>

5-(2,4,7-trimethyl-1,3-dioxo-1,2,3,4-tetrahydroisoquinolin-4-yl)pentanenitrile (4f). ${ }^{4}$ Following the General procedure $B$, the product was purified by flash chromatography on silica gel (petroleum ether/EtOAc, 20:1 to $5: 1$ ), $35.5 \mathrm{mg}$, yield: $50 \%$, colorless oil. ${ }^{1} \mathrm{H}$ $\operatorname{NMR}\left(\mathrm{CDCl}_{3}, 300 \mathrm{MHz}\right): \delta 8.02(\mathrm{~s}, 1 \mathrm{H}), 7.44(\mathrm{~d}, J=7.9 \mathrm{~Hz}, 1 \mathrm{H}), 7.27(\mathrm{~d}, J=8.0 \mathrm{~Hz}, 1 \mathrm{H}), 3.34(\mathrm{~s}$, $3 \mathrm{H}), 2.40(\mathrm{~s}, 3 \mathrm{H}), 2.33-2.23(\mathrm{~m}, 1 \mathrm{H}), 2.20-2.15(\mathrm{~m}, 2 \mathrm{H}), 1.91-1.81(\mathrm{~m}, 1 \mathrm{H}), 1.56-1.41(\mathrm{~m}, 5 \mathrm{H})$, 1.07-0.84 (m, 2H); ${ }^{13} \mathrm{C}\left\{{ }^{1} \mathrm{H}\right\} \mathrm{NMR}\left(\mathrm{CDCl}_{3}, 75 \mathrm{MHz}\right): \delta 176.6,164.5,140.1,137.5,135.3,129.0$, 125.0, 124.6, 119.3, 47.3, 41.7, 29.8, 27.2, 25.3, 24.5, 21.0, 16.8 .<smiles></smiles>

\section{5-(6,7-dichloro-2,4-dimethyl-1,3-dioxo-1,2,3,4-tetrahydroisoquinolin-4-}

yl)pentanenitrile $(\mathbf{4 g})$. Following the General procedure $B$, the product was purified by flash chromatography on silica gel (petroleum ether/EtOAc, 20:1 to 10:1), $44.3 \mathrm{mg}$, yield: $52 \%$, colorless oil. ${ }^{1} \mathrm{H}$ NMR $\left(\mathrm{CDCl}_{3}, 300 \mathrm{MHz}\right): \delta 8.28(\mathrm{~s}, 1 \mathrm{H}), 7.48(\mathrm{~s}, 1 \mathrm{H}), 3.35(\mathrm{~s}, 3 \mathrm{H})$, 2.35-2.19 $(\mathrm{m}, 3 \mathrm{H}), 1.88-1.78(\mathrm{~m}, 1 \mathrm{H}), 1.59-1.48(\mathrm{~m}, 5 \mathrm{H}), 1.10-0.84(\mathrm{~m}, 2 \mathrm{H}) ;{ }^{13} \mathrm{C}\left\{{ }^{1} \mathrm{H}\right\} \mathrm{NMR}\left(\mathrm{CDCl}_{3}\right.$, $75 \mathrm{MHz}$ ): $\delta 175.3,162.5,142.5,139.1,132.6,130.7,127.5,124.7,119.1,47.5,41.8,29.5,27.5$, 25.2, 24.4, 16.8. IR (ATR): 2943, 2869, 2246, 1714, 1665, 1594, 1426, $781 \mathrm{~cm}^{-1}$. HRMS (ESI) m/z: $[\mathrm{M}+\mathrm{Na}]^{+}$Calcd for $\mathrm{C}_{16} \mathrm{H}_{16} \mathrm{Cl}_{2} \mathrm{~N}_{2} \mathrm{NaO}_{2}$ 361.0481; Found 361.0485. 


\section{Diversity of the Products}

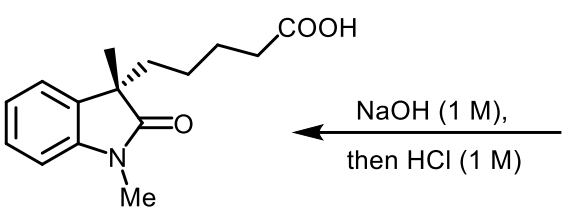

5b, $90 \%$

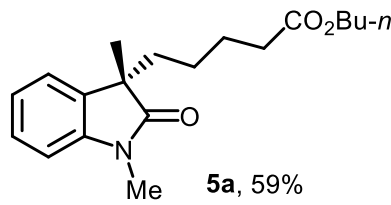
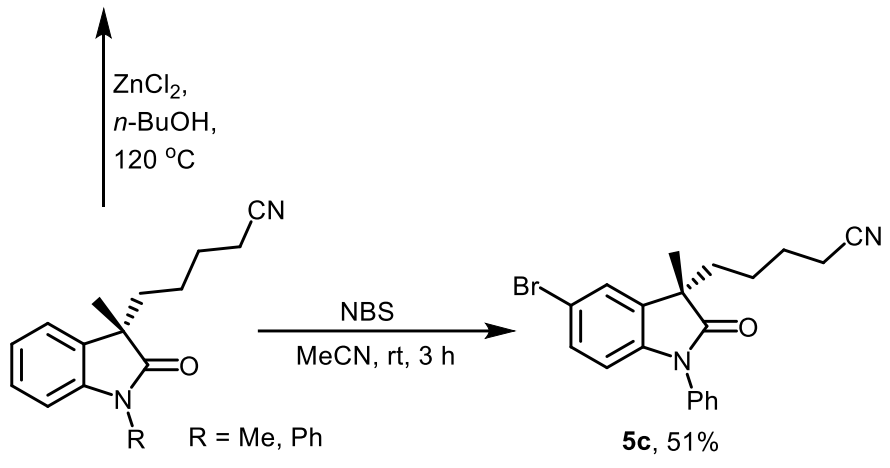

$3 a$ or $3 f$

\section{(a) Alcoholysis with $\boldsymbol{n}$-BuOH}

To a $10 \mathrm{ml}$ oven-dried Schlenk tube was charged with $3 a$ ( $0.2 \mathrm{mmol}, 1.0$ equiv), $\mathrm{ZnCl}_{2}$ ( $0.2 \mathrm{mmol}, 1.0$ equiv). $n$-BuOH $(1 \mathrm{~mL}$ ) was then injected into the tube by syringe. The resulting mixture was stirred vigorously for $24 \mathrm{~h}$ at $120^{\circ} \mathrm{C}$. Upon completion of the reaction, the mixture was diluted with EtOAc. The solvent was then removed under vacuo. The reaction was then extracted with EtOAc for three times. The combined organic extracts were washed with brine, dried with $\mathrm{MgSO}_{4}$, filtrated and concentrated in vacuo. The product was purified by flash column chromatography $(P E / E t O A C=20: 1)$ to yield $\mathbf{5 a}$ as a colorless oil.

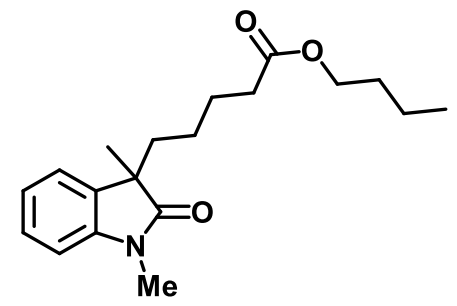

butyl 5-(1,3-dimethyl-2-oxoindolin-3-yl)pentanoate (5a). $37.5 \mathrm{mg}$, yield: 59\%, colorless oil. ${ }^{1} \mathrm{H}$ NMR $\left(\mathrm{CDCl}_{3}, 300 \mathrm{MHz}\right): \delta$ 7.27-7.22 (m, $\left.1 \mathrm{H}\right), 7.14(\mathrm{~d}, J=7.0 \mathrm{~Hz}, 1 \mathrm{H}), 7.05(\mathrm{t}, J=7.4 \mathrm{~Hz}$, $1 \mathrm{H}), 6.82(\mathrm{~d}, J=7.7 \mathrm{~Hz}, 1 \mathrm{H}), 3.98(\mathrm{t}, J=6.6 \mathrm{~Hz}, 2 \mathrm{H}), 3.19(\mathrm{~s}, 3 \mathrm{H}), 2.18-2.13(\mathrm{~m}, 2 \mathrm{H}), 1.94-1.84$ $(\mathrm{m}, 1 \mathrm{H}), 1.77-1.67(\mathrm{~m}, 1 \mathrm{H}), 1.57-1.48(\mathrm{~m}, 4 \mathrm{H}), 1.33-1.24(\mathrm{~m}, 5 \mathrm{H}), 1.08-0.87(\mathrm{~m}, 5 \mathrm{H}) ;{ }^{13} \mathrm{C}\left\{{ }^{1} \mathrm{H}\right\}$ NMR (CDCl $\left.{ }_{3}, 75 \mathrm{MHz}\right): \delta$ 180.7, 173.7, 143.4, 134.1, 127.8, 122.6, 122.5, 108.0, 64.2, 48.4, 38.1, 34.1, 30.7, 26.2, 25.1, 24.1, 23.9, 19.2, 13.8. IR (ATR): 2960, 2933, 2870, 1711, 1613, 1493, $1377,1247,751 \mathrm{~cm}^{-1}$. HRMS (ESI) m/z: [M+H] $]^{+}$Calcd for $\mathrm{C}_{19} \mathrm{H}_{28} \mathrm{NO}_{3} 318.2064$; Found 318.2067.

\section{(b) Hydrolysis}

To a solution of aqueous $\mathrm{NaOH}(1.0 \mathrm{M}, 1.3 \mathrm{~mL}$ ) was added nitrile $3 a(61 \mathrm{mg}, 0.25$ $\mathrm{mmol}$ ). The flask was placed into a preheated $100{ }^{\circ} \mathrm{C}$ (oil bath) and stirred for $24 \mathrm{~h}$. After cooling down to room temperature and the aqueous phase was acidified with 
concentrated $\mathrm{HCl}(\mathrm{pH}=2)$, the mixture was extracted with EtOAc $(3 \times 10 \mathrm{~mL})$ and the combined organic layers were washed with brine, dried over anhydrous $\mathrm{MgSO}_{4}$, filtered, and concentrated under reduced pressure by rotary evaporation. Purification by flash column chromatography on silica gel $(P E / E t O A c=5: 1)$ afforded the title compound as a white solid.

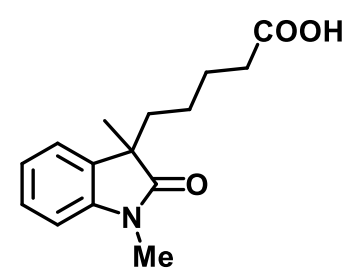

5-(1,3-dimethyl-2-oxoindolin-3-yl)pentanoic acid (5b). ${ }^{3} 71.72 \mathrm{mg}$, yield: $90 \%$, white solid. ${ }^{1} \mathrm{H}$ NMR $\left(\mathrm{CDCl}_{3}, 300 \mathrm{MHz}\right): \delta 9.21(\mathrm{~s}, 1 \mathrm{H}), 7.26-7.22(\mathrm{~m}, 1 \mathrm{H}), 7.14(\mathrm{~d}, J=7.1 \mathrm{~Hz}, 1 \mathrm{H}), 7.07-$ $7.02(\mathrm{~m}, 1 \mathrm{H}), 6.83(\mathrm{~d}, J=7.7 \mathrm{~Hz}, 1 \mathrm{H}), 3.19(\mathrm{~s}, 3 \mathrm{H}), 2.18(\mathrm{t}, J=7.5 \mathrm{~Hz}, 2 \mathrm{H}), 1.95-1.85(\mathrm{~m}, 1 \mathrm{H})$, 1.77-1.67 (m, 1H), 1.53-1.42 (m, 2H), $1.32(\mathrm{~s}, 3 \mathrm{H}), 1.10-0.96(\mathrm{~m}, 1 \mathrm{H}), 0.91-0.79(\mathrm{~m}, 1 \mathrm{H}) ;{ }^{13} \mathrm{C}\left\{{ }^{1} \mathrm{H}\right\}$ $\operatorname{NMR}\left(\mathrm{CDCl}_{3}, 75 \mathrm{MHz}\right): \delta$ 180.9, 178.7, 143.1, 133.9, 127.7, 122.7, 122.4, 108.1, 48.4, 37.9, 33.7, $26.2,24.7,23.9,23.8$.

\section{(c) Bromination}

To a Schlenktube were added $\mathbf{3 f}$ (1 mmol, 76.7mg), $\mathrm{N}$-bromosuccinimide (NBS) (1.1 equiv., $39.2 \mathrm{mg}$ ) and $\mathrm{CH}_{3} \mathrm{CN}(2 \mathrm{~mL})$. The reaction mixture was stirred at room temperature for $3 \mathrm{~h}$. Concentration of the reaction mixture in vacuo followed by silica gel chromatography eluting with PE/EtOAc $(10: 1)$ afforded the $\mathbf{5 c}$ as a white solid.<smiles>CC1(CCCCC#N)C(=O)N(c2ccccc2)c2ccc(Br)cc21</smiles>

5-(5-bromo-3-methyl-2-oxo-1-phenylindolin-3-yl)pentanenitrile (5c). $38.7 \mathrm{mg}$, yield: $51 \%$, white solid. ${ }^{1} \mathrm{H} \mathrm{NMR}\left(\mathrm{CDCl}_{3}, 300 \mathrm{MHz}\right): \delta 7.55-7.49(\mathrm{~m}, 2 \mathrm{H}), 7.43-7.30(\mathrm{~m}, 5 \mathrm{H}), 6.72(\mathrm{~d}, J$ $=8.2 \mathrm{~Hz}, 1 \mathrm{H}), 2.26(\mathrm{t}, J=7.2 \mathrm{~Hz}, 2 \mathrm{H}), 2.08-1.98(\mathrm{~m}, 1 \mathrm{H}), 1.87-1.77(\mathrm{~m}, 1 \mathrm{H}), 1.65-1.54(\mathrm{~m}, 2 \mathrm{H})$, $1.47(\mathrm{~s}, 3 \mathrm{H}), 1.31-1.12(\mathrm{~m}, 2 \mathrm{H}) ;{ }^{13} \mathrm{C}\left\{{ }^{1} \mathrm{H}\right\} \mathrm{NMR}\left(\mathrm{CDCl}_{3}, 75 \mathrm{MHz}\right): \delta 179.1,142.2,135.5,134.1$, 130.8, 129.8, 128.3, 126.4, 126.0, 119.4, 115.9, 111.0, 48.5, 37.9, 25.4, 24.2, 23.8, 17.0. IR (ATR): 3065, 2933, 2865, 2246, 1719, 1601, 1502, 1474, 1359, 1244, $761 \mathrm{~cm}^{-1}$. HRMS (ESI) m/z: $[\mathrm{M}+\mathrm{Na}]^{+}$Calcd for $\mathrm{C}_{20} \mathrm{H}_{19} \mathrm{BrN}_{2} \mathrm{NaO}$ 405.0573; Found 405.0577. 


\section{Mechanism Study}

\section{Radial capture experiments}<smiles>C=C(C)C(=O)N(C)c1ccccc1</smiles>

$1 \mathrm{a}$<smiles>CC(C)(C)ON=C1CCC1</smiles>

$2 a$
TEMPO ( 1.50 equiv.)

DMSO, $\mathrm{N}_{2}, 110^{\circ} \mathrm{C}, 24 \mathrm{~h}$

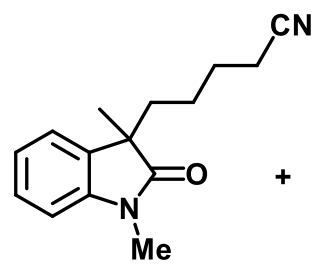

3a, ND

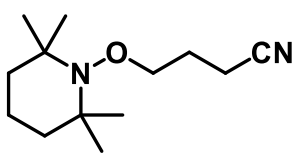

$6 a$, yield: $35 \%$

Procedures:

\section{(a) TEMPO}

$\mathrm{N}$-arylacrylamide $1 \mathrm{a}$ ( $0.375 \mathrm{mmol}, 52.5 \mathrm{mg}, 1.5$ equiv.), TEMPO (58.6 mg, 1.5 equiv.), DMSO $(0.5 \mathrm{~mL})$ were weighed into a Schlenk tube. The reaction vessel was capped and subjected to three vacuum-purge/nitrogen-flush cycles. Then $2 \mathrm{a}(0.25 \mathrm{mmol}, 44.4 \mu \mathrm{L}$, 1.0 equiv.) was added through the side-arm by syringe. The reaction was stirred under argon at $110{ }^{\circ} \mathrm{C}$ for $24 \mathrm{~h}$. After reaction, the mixture was extracted by EtOAc, then the solvent was evaporated under reduced pressure and it was submitted to ${ }^{1} \mathrm{H}$ NMR. No product was detected by NMR.

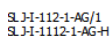

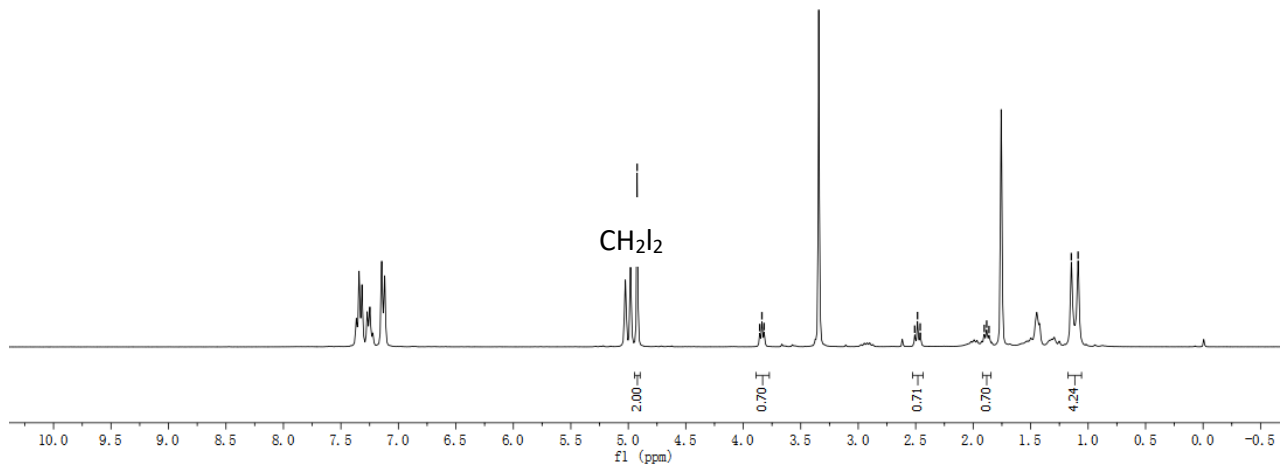


(b) (1-cyclopropylvinyl)benzene<smiles>C=C(C)C(=O)N(C)c1ccccc1</smiles>

$1 \mathrm{a}$<smiles>CC(C)(C)ON=C1CCC1</smiles>

$2 \mathrm{a}$

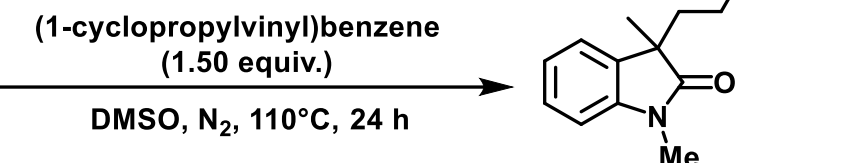

3a, yield: $16 \%$

$N$-arylacrylamide 1a ( $0.375 \mathrm{mmol}, 52.5 \mathrm{mg}, 1.5$ equiv.), (1-cyclopropylvinyl)benzene (54.1 mg, 1.5 equiv.), DMSO $(0.5 \mathrm{~mL})$ were weighed into a Schlenk tube. The reaction vessel was capped and subjected to three vacuum-purge/nitrogen-flush cycles. Then 2a $(0.25 \mathrm{mmol}, 44.4 \mu \mathrm{L}, 1.0$ equiv.) was added through the side-arm by syringe. The reaction was stirred under argon at $110{ }^{\circ} \mathrm{C}$ for $24 \mathrm{~h}$. After reaction, the mixture was extracted by EtOAc, then the solvent was evaporated under reduced pressure and it was submitted to ${ }^{1} \mathrm{H}$ NMR. The product 3 a was got in $16 \%$ NMR yield.

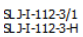
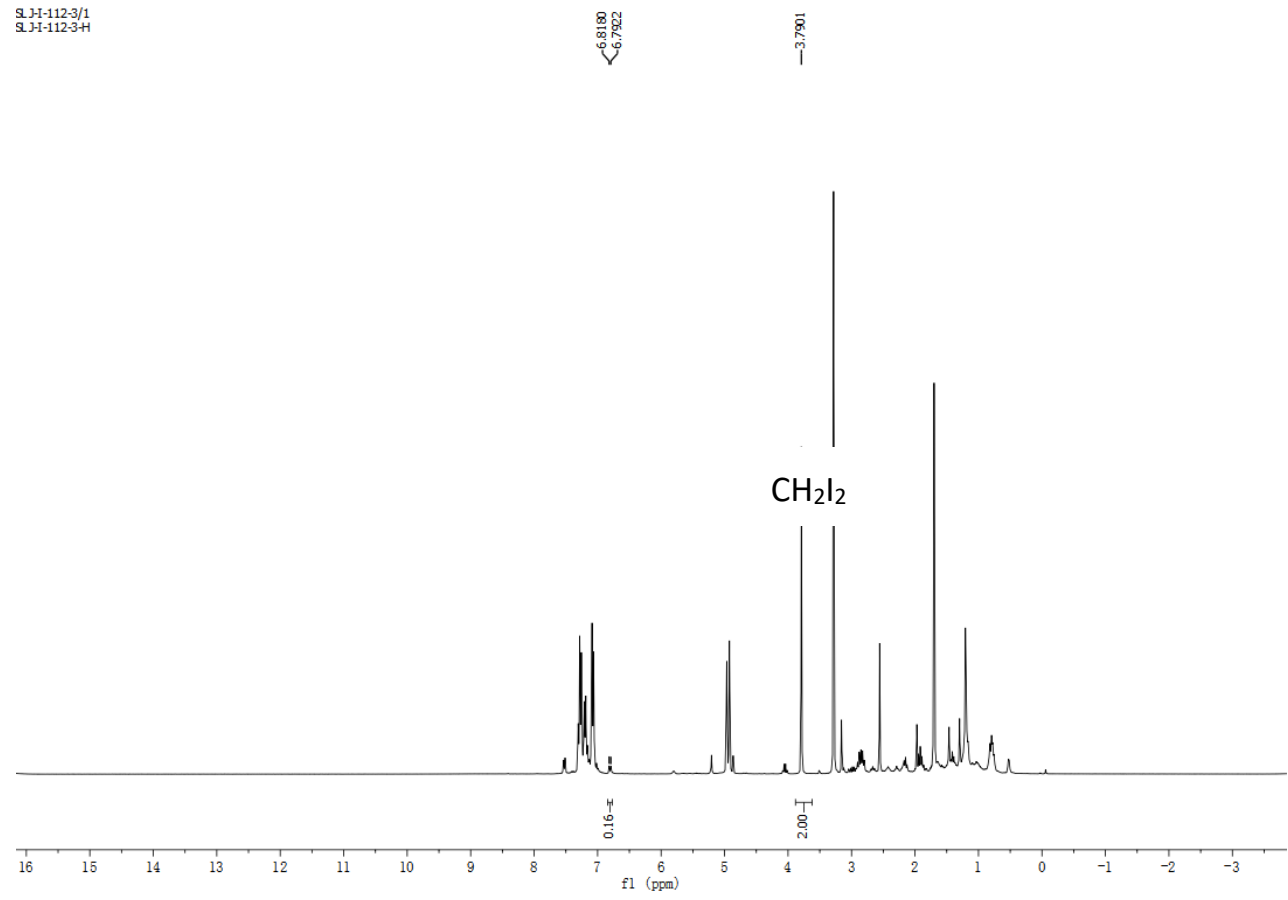

(c) 1,1-Diphenylethylene<smiles>C=C(C)C(=O)N(C)c1ccccc1</smiles>

$1 \mathrm{a}$<smiles>O=C(OCc1ccccc1)ON=C1CCC1</smiles>

$2 \mathrm{a}$

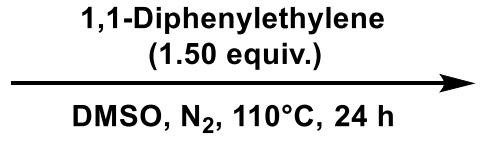

DMSO, $\mathrm{N}_{2}, 110^{\circ} \mathrm{C}, 24 \mathrm{~h}$<smiles>CN1C(=O)C(C)(C)c2ccccc21</smiles>

3a, yield: $8 \%$

$N$-arylacrylamide 1a (0.375 mmol, 52.5 mg, 1.5 equiv.), 1,1-Diphenylethylene 
(66.2 $\mu \mathrm{L}, 1.5$ equiv.), DMSO $(0.5 \mathrm{~mL})$ were weighed into a Schlenk tube. The reaction vessel was capped and subjected to three vacuum-purge/nitrogen-flush cycles. Then 2a ( $0.25 \mathrm{mmol}, 44.4 \mu \mathrm{L}, 1.0$ equiv.) was added through the side-arm by syringe. The reaction was stirred under argon at $110{ }^{\circ} \mathrm{C}$ for $24 \mathrm{~h}$. After reaction, the mixture was extracted by EtOAc, then the solvent was evaporated under reduced pressure and it was submitted to ${ }^{1} \mathrm{H}$ NMR. The product 3 a was got in $8 \%$ NMR yield.

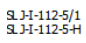
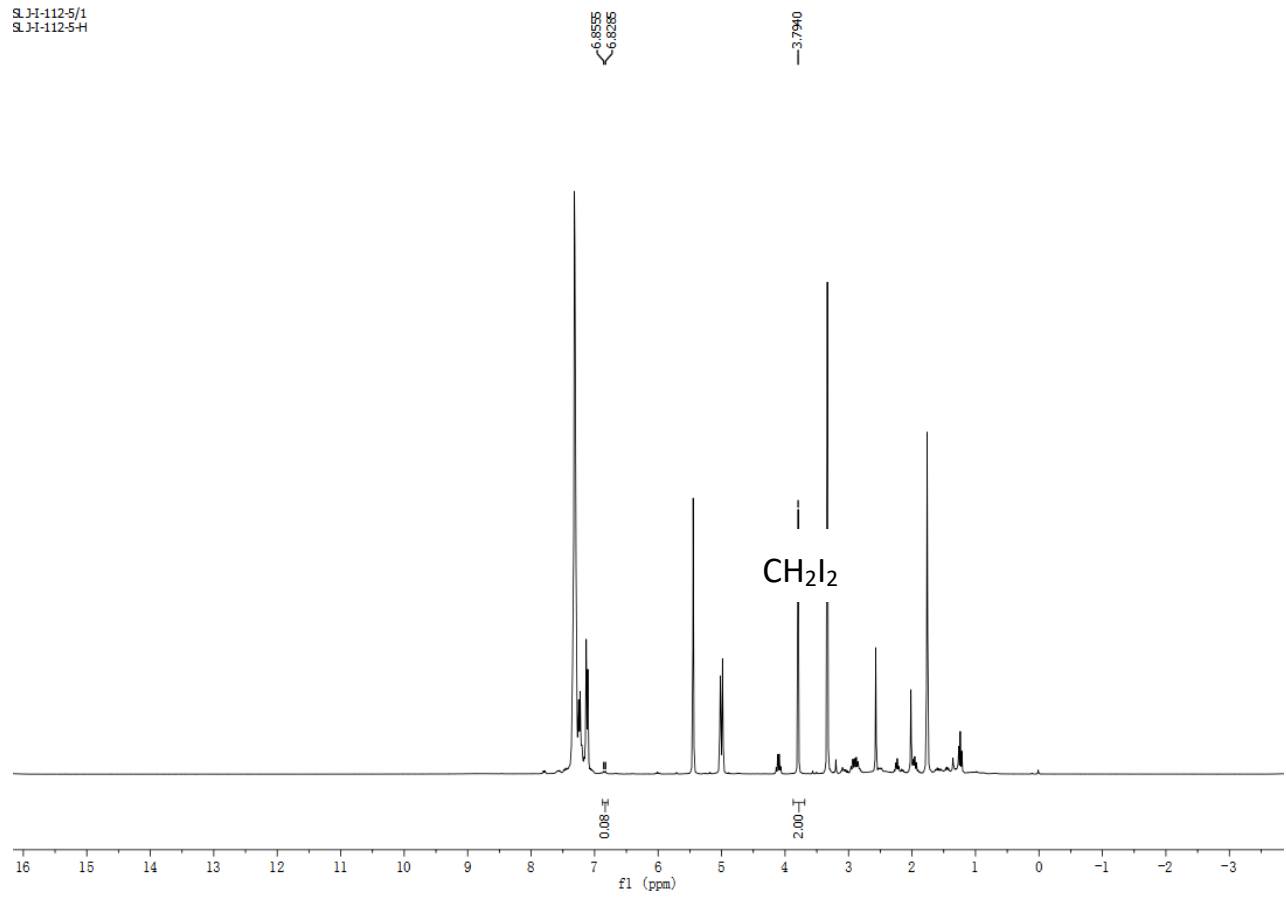

(d) Under $\mathrm{O}_{2}$

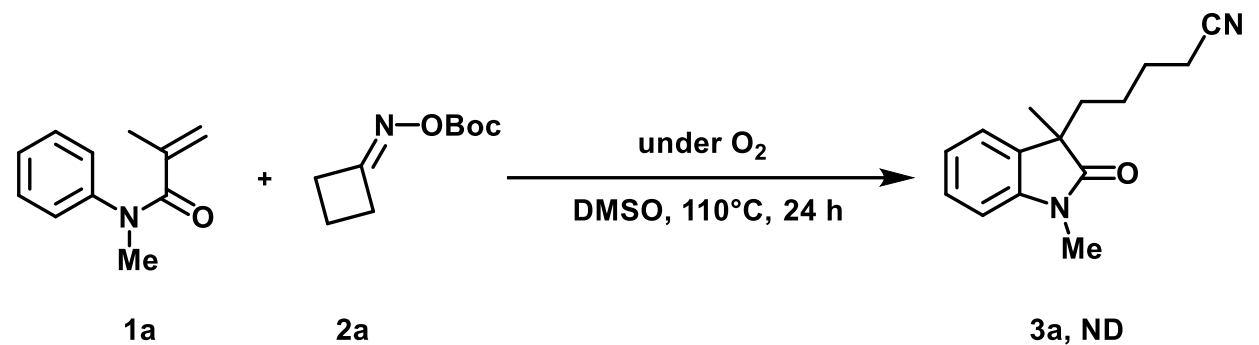

$\mathrm{N}$-arylacrylamide 1a (0.375 mmol, $52.5 \mathrm{mg}, 1.5$ equiv.), DMSO $(0.5 \mathrm{~mL})$ were weighed into a Schlenk tube. The reaction vessel was capped and subjected to $\mathrm{O}_{2}$ (via $\mathrm{O}_{2}$ balloon). Then $2 \mathrm{a}(0.25 \mathrm{mmol}, 44.4 \mu \mathrm{L}, 1.0$ equiv.) was added through the side-arm by syringe. The reaction was stirred under $\mathrm{O}_{2}$ at $110{ }^{\circ} \mathrm{C}$ for $24 \mathrm{~h}$. After reaction, the mixture was extracted by EtOAc, then the solvent was evaporated under reduced pressure and it was submitted to ${ }^{1} \mathrm{H}$ NMR. No product was detected by NMR. 
(e) 1,1-Diphenylethylene<smiles>C=C(c1ccccc1)c1ccccc1</smiles><smiles>CC(C)(C)ON=C1CCC1</smiles>

2a

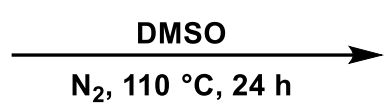

$\mathrm{N}_{2}, 110^{\circ} \mathrm{C}, 24 \mathrm{~h}$

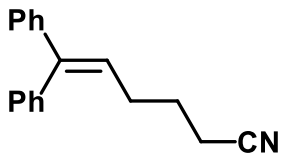

6b, yield: $5 \%$

1,1-Diphenylethylene ( $0.375 \mathrm{mmol}, 66.2 \mu \mathrm{L}, 1.5$ equiv.), DMSO $(0.5 \mathrm{~mL})$ were weighed into a Schlenk tube. The reaction vessel was capped and subjected to three vacuumpurge/ nitrogen-flush cycles. Then $2 \mathrm{a}(0.25 \mathrm{mmol}, 44.4 \mu \mathrm{L}, 1.0$ equiv.) was added through the side-arm by syringe. The reaction was stirred under argon at $110{ }^{\circ} \mathrm{C}$ for 24 h. After reaction, the mixture was extracted by EtOAc, then the solvent was evaporated under reduced pressure and it was submitted to ${ }^{1} \mathrm{H}$ NMR. The product $6 \mathbf{6}$ was got in $5 \%$ NMR yield.

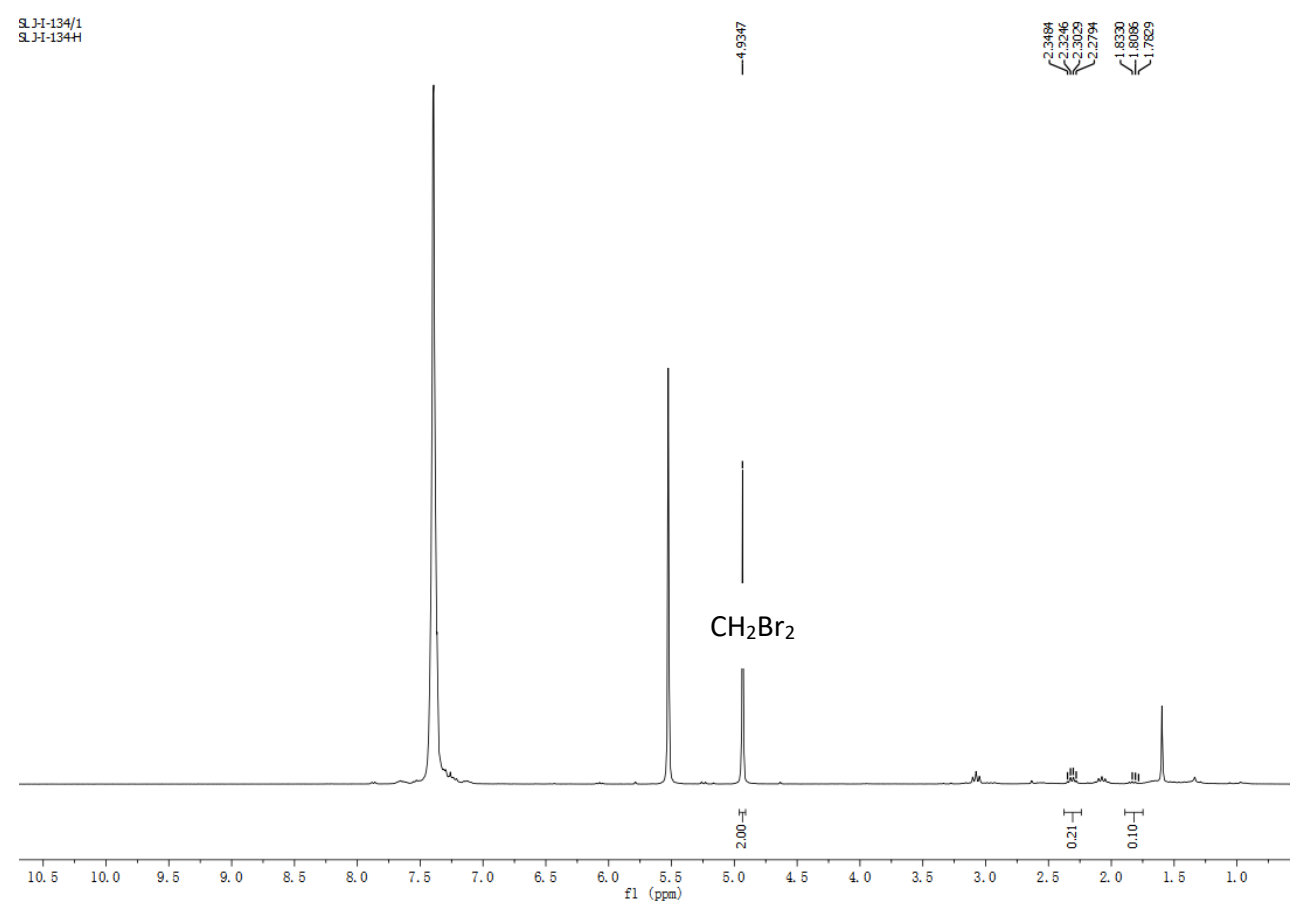




\section{References}

(1) Kilaru, P.; Acharya, S. P.; Zhao, P. A tethering directing group strategy for ruthenium-catalyzed intramolecular alkene hydroarylation. Chem. Commun. 2018, 54, 924-927.

(2) a) Liu, Z.; Shen, H.; Xiao, H.; Wang, Z.; Zhu, L.; Li, C. Copper-Catalyzed Ring-Opening Radical Trifluoromethylation of Cycloalkanone Oximes. Org. Lett. 2019, 21, 52015205; b) Sietmann, J.; Ong, M.; Mück-Lichtenfeld, C.; Daniliuc, C. G.; Wiest, J. M. Desymmetrization of Prochiral Cyclobutanones via Nitrogen Insertion: A Concise Route to Chiral $\gamma$-Lactams. Angew. Chem. Int. Ed. 2021, 60, 9719-9723.

(3) Wu, J.; Zhang, J.-Y.; Gao, P.; Xu, S.-L.; Guo, L.-N. Copper-catalyzed redox-neutral cyanoalkylarylation of activated alkenes with cyclobutanone oxime esters. J. Org. Chem. 2018, 83, 1046-1055.

(4) Wang, Z.; Yan, X.; He, X.; Yan, X.; Li, X.; Xu, X. Iminyl-Radical-Mediated Cyanoalkylarylation of Activated Alkenes Enabled by Silver-Catalyzed Decarboxylation of $\alpha$-Imino Oxy Acids. Synlett 2020, 31, 809-812. 
NMR Spectra Images of Products

${ }^{1} \mathbf{H}$ NMR of compound $3 \mathbf{3 a}\left(300 \mathrm{MHz}\right.$ in $\left.\mathrm{CDCl}_{3}\right)$

s. $-\mathrm{I}-\mathrm{-}-\mathrm{0}-1 / 1$

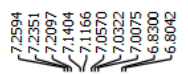

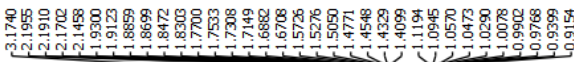

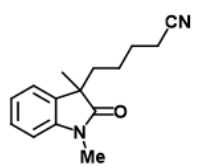

3a

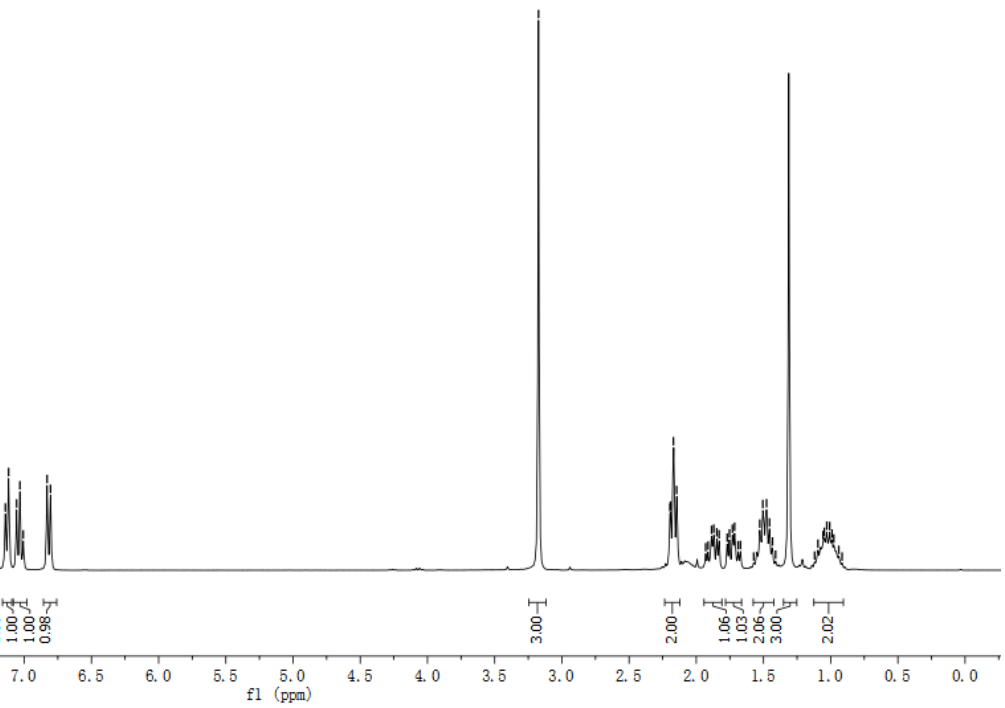

${ }^{13} \mathrm{C}\left\{{ }^{1} \mathrm{H}\right\}$ NMR of compound $\mathbf{3 a}\left(75 \mathrm{MHz}\right.$ in $\left.\mathrm{CDCl}_{3}\right)$
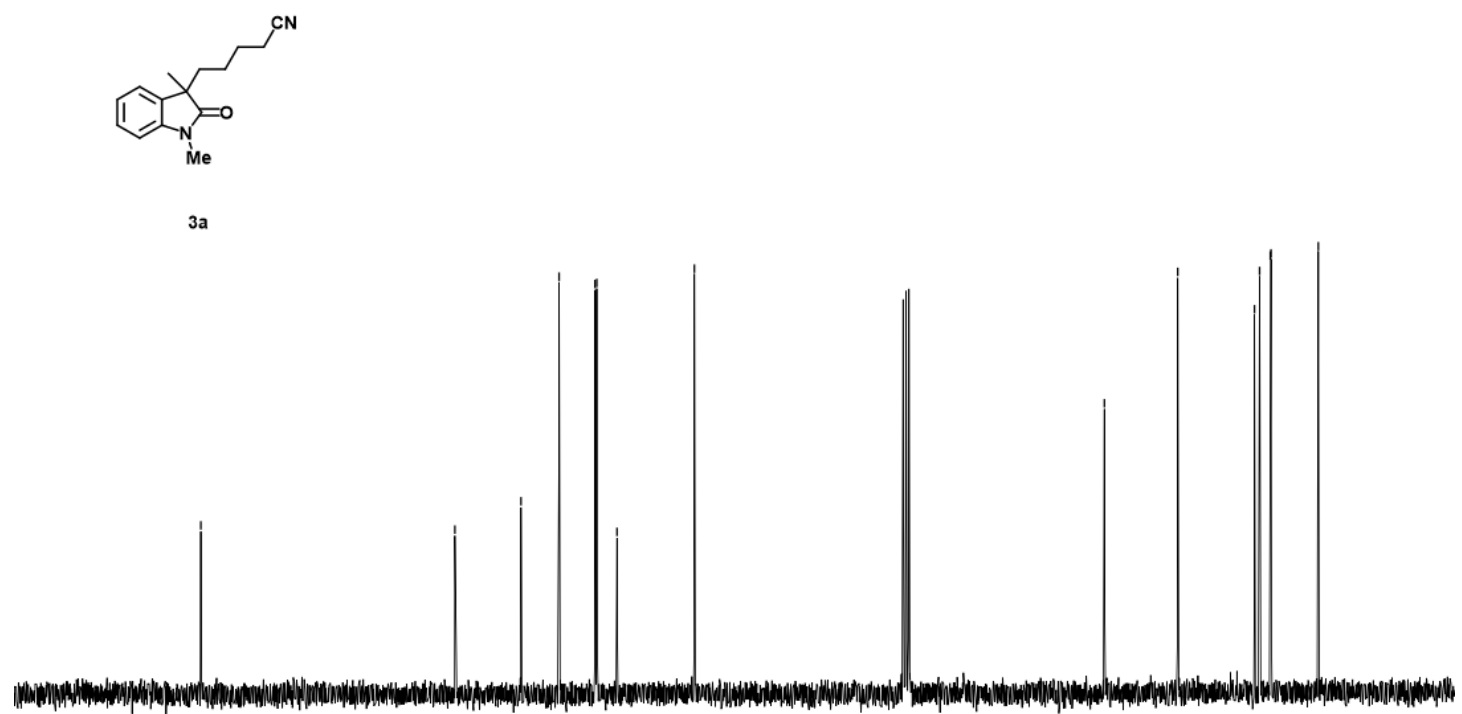
${ }^{1} \mathbf{H}$ NMR of compound $\mathbf{3 b}\left(300 \mathrm{MHz}\right.$ in $\left.\mathrm{CDCl}_{3}\right)$

sji-80-3/1

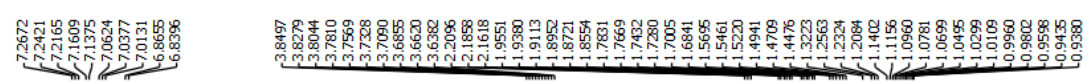

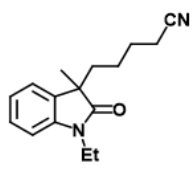

3b

${ }^{13} \mathbf{C}\left\{{ }^{1} \mathrm{H}\right\}$ NMR of compound $\mathbf{3 b}\left(75 \mathrm{MHz}\right.$ in $\left.\mathrm{CDCl}_{3}\right)$

$\mathrm{sj}-\mathrm{-}-80-3-\mathrm{c} / 1$

亭

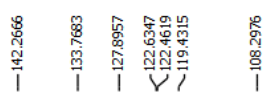

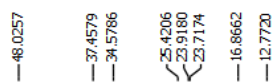

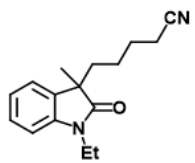

3b

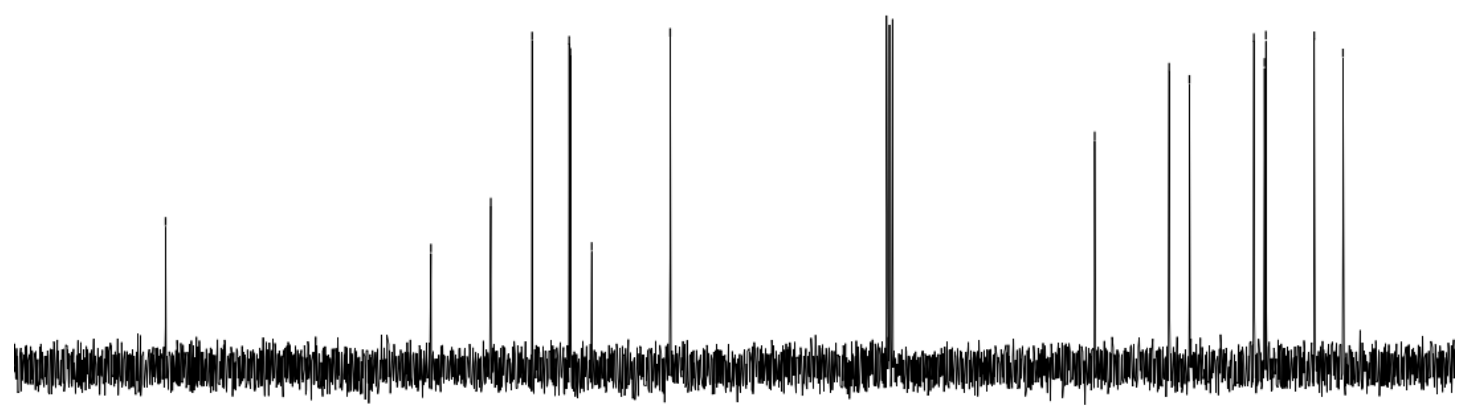

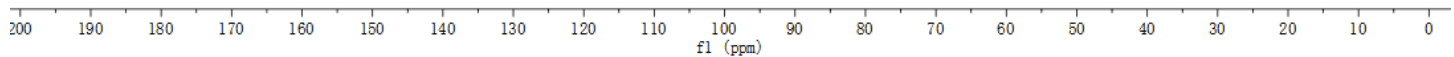


${ }^{1} \mathbf{H}$ NMR of compound $3 \mathbf{c}\left(300 \mathrm{MHz}\right.$ in $\left.\mathrm{CDCl}_{3}\right)$

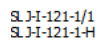

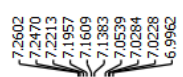

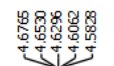

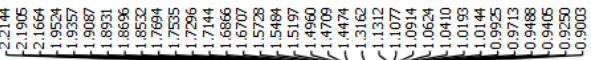

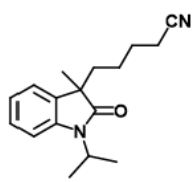

$3 c$

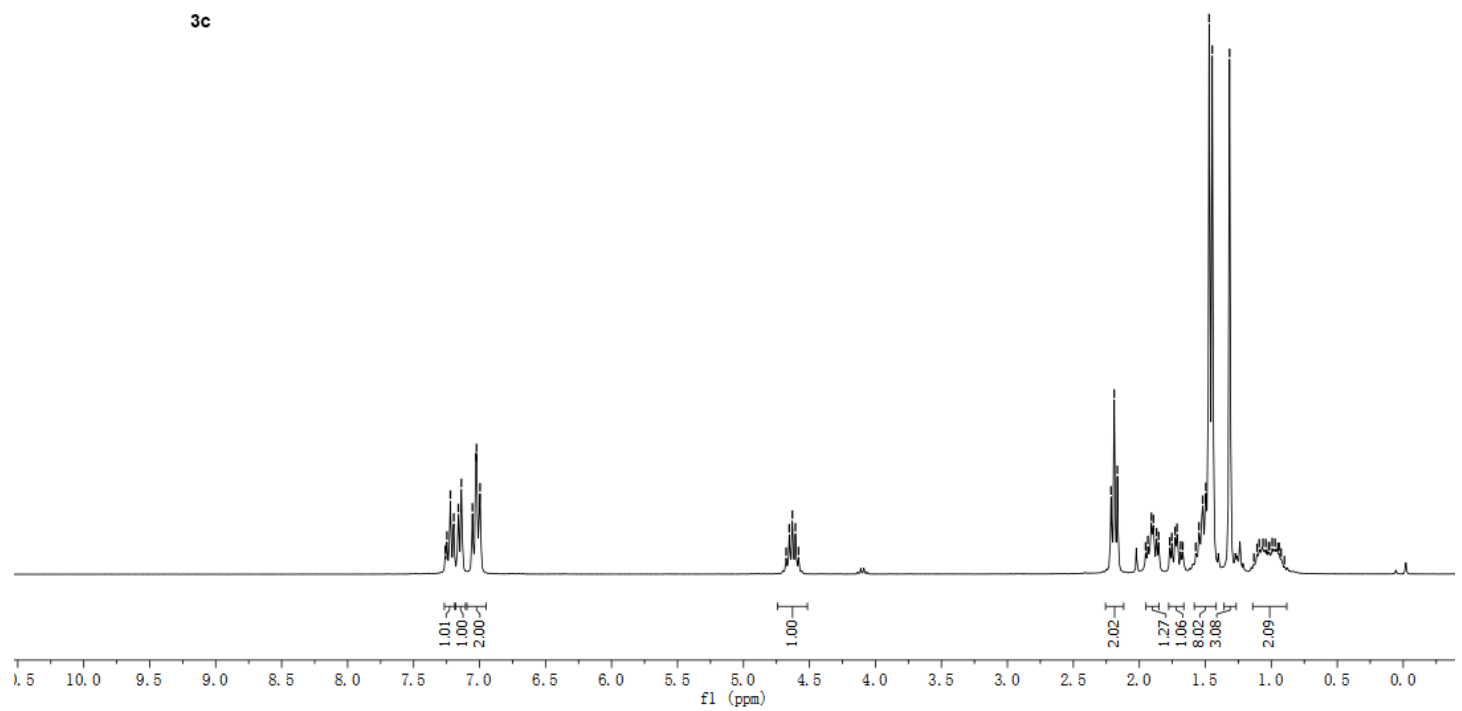

${ }^{13} \mathrm{C}\left\{{ }^{1} \mathrm{H}\right\}$ NMR of compound $3 \mathrm{c}\left(75 \mathrm{MHz}\right.$ in $\left.\mathrm{CDCl}_{3}\right)$

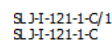

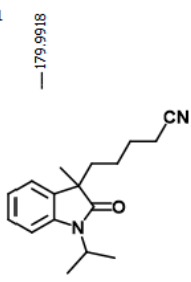

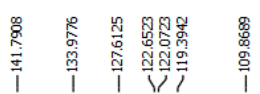

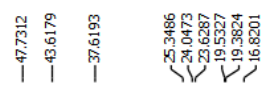

$3 c$

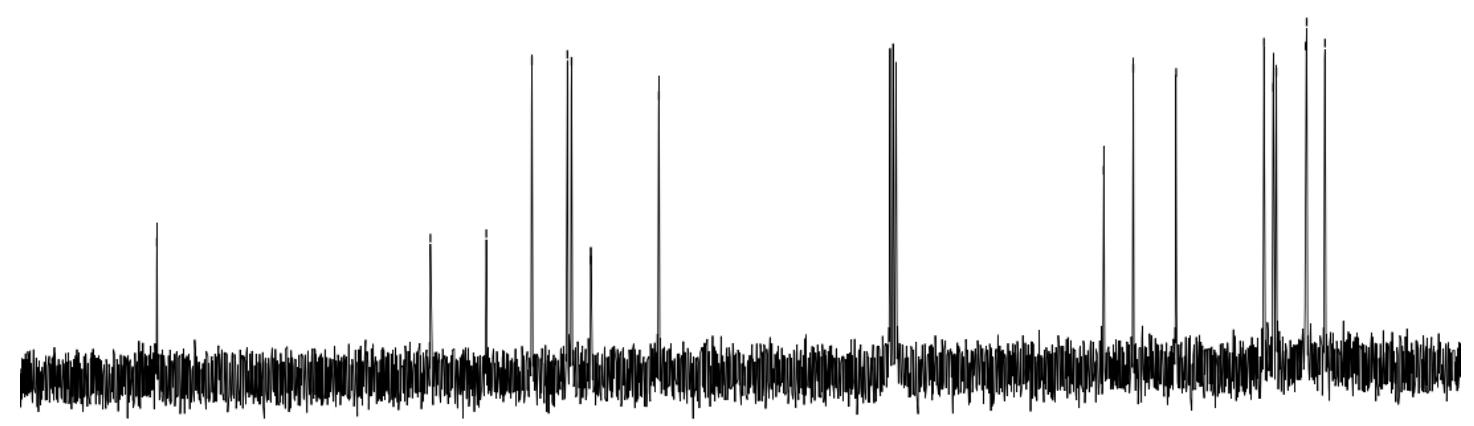

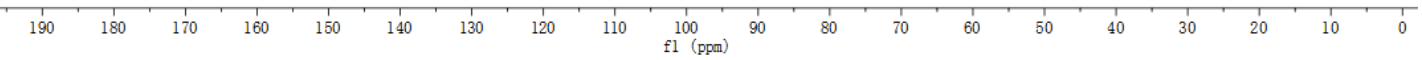


${ }^{1} \mathbf{H}$ NMR of compound $\mathbf{3 d}\left(300 \mathrm{MHz}\right.$ in $\left.\mathrm{CDCl}_{3}\right)$

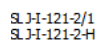
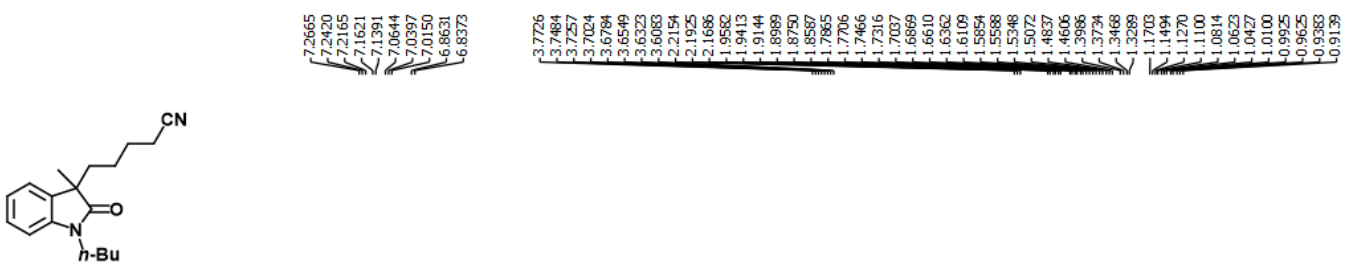

$3 d$

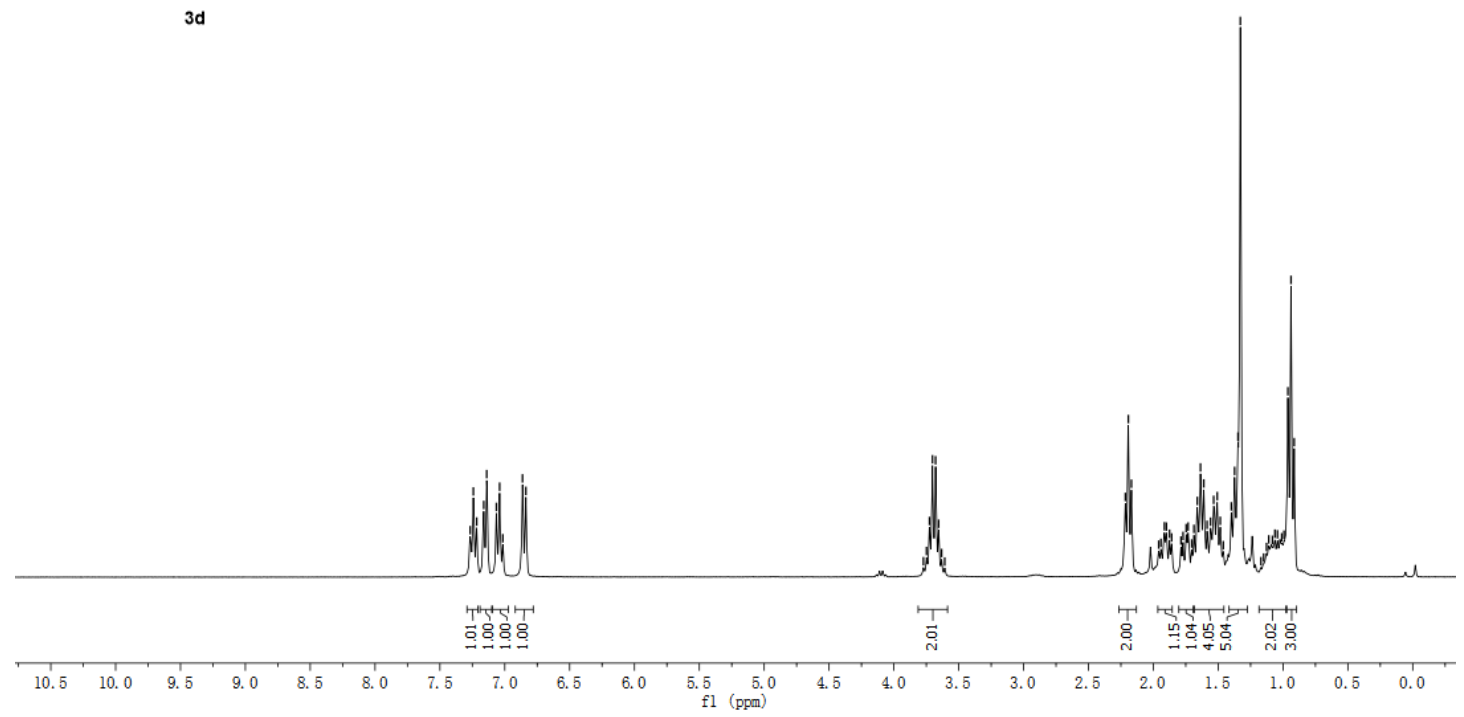

${ }^{13} \mathrm{C}\left\{{ }^{1} \mathrm{H}\right\}$ NMR of compound $\mathbf{3 d}\left(75 \mathrm{MHz}\right.$ in $\left.\mathrm{CDCl}_{3}\right)$

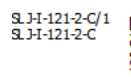

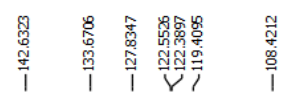

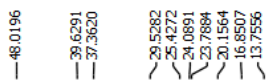

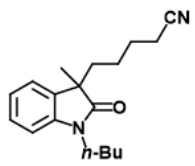

3d

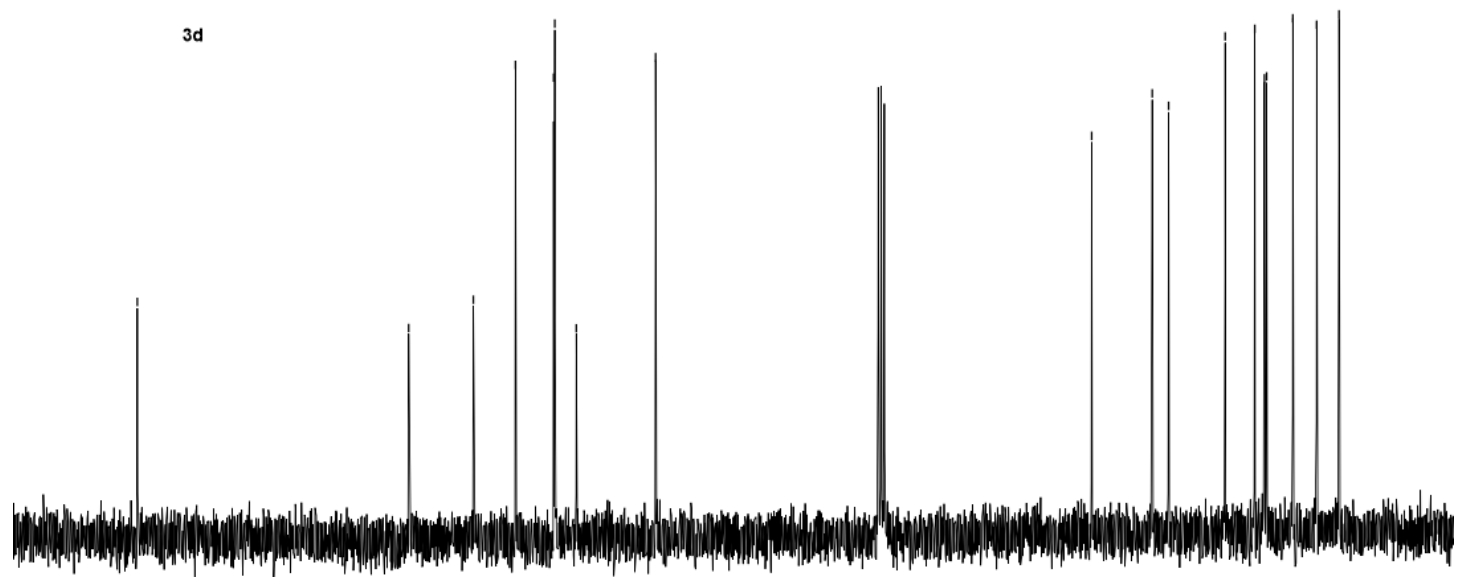

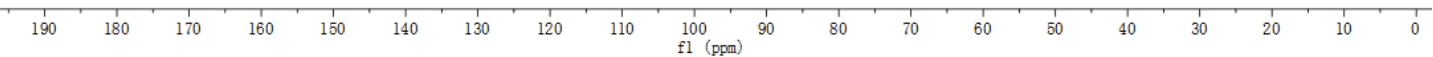


${ }^{1} \mathbf{H}$ NMR of compound $3 e\left(300 \mathrm{MHz}\right.$ in $\left.\mathrm{CDCl}_{3}\right)$

sjj-80-5/1
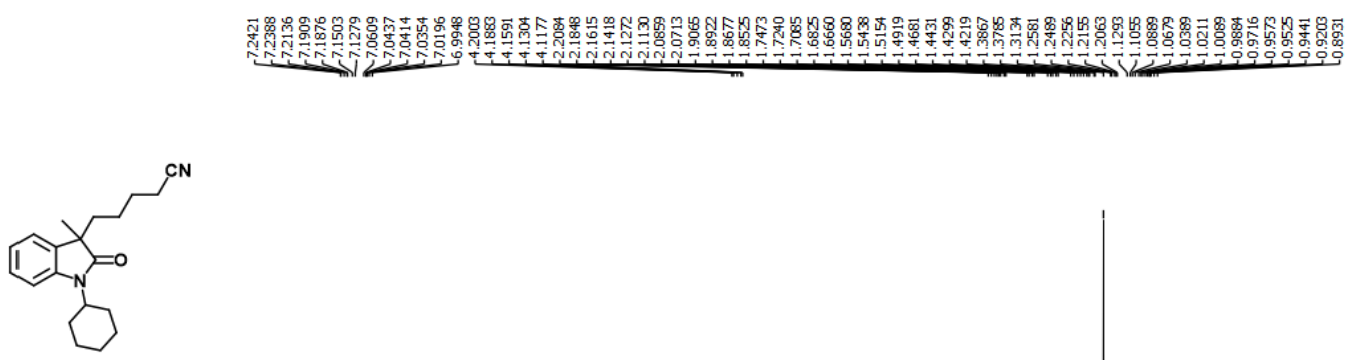

3e

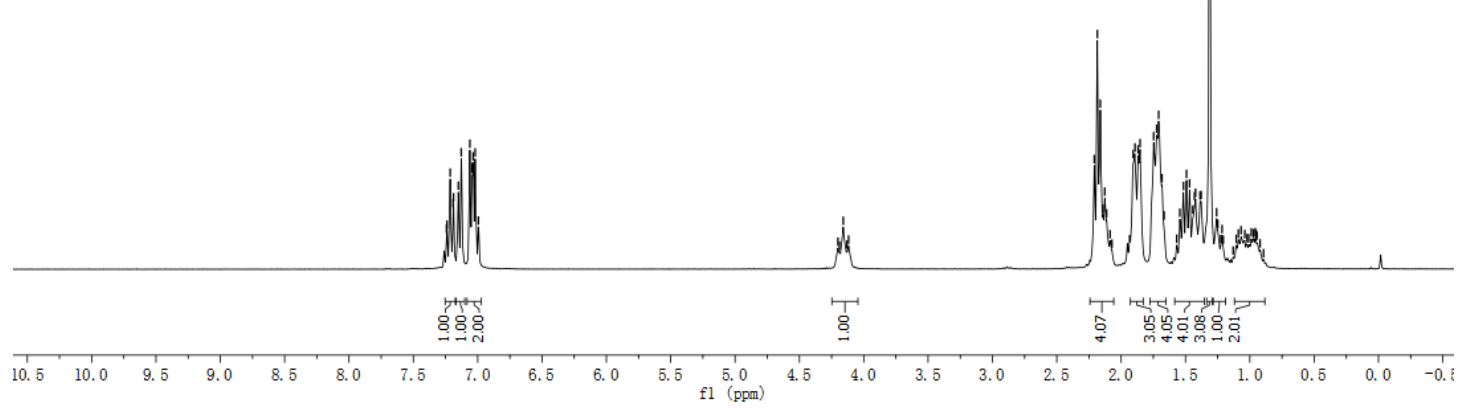

${ }^{13} \mathrm{C}\left\{{ }^{1} \mathrm{H}\right\}$ NMR of compound $3 e\left(75 \mathrm{MHz}\right.$ in $\left.\mathrm{CDCl}_{3}\right)$

dj $-80-5-c / 2$

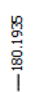

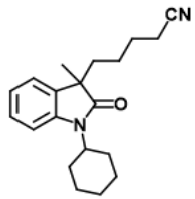

3e
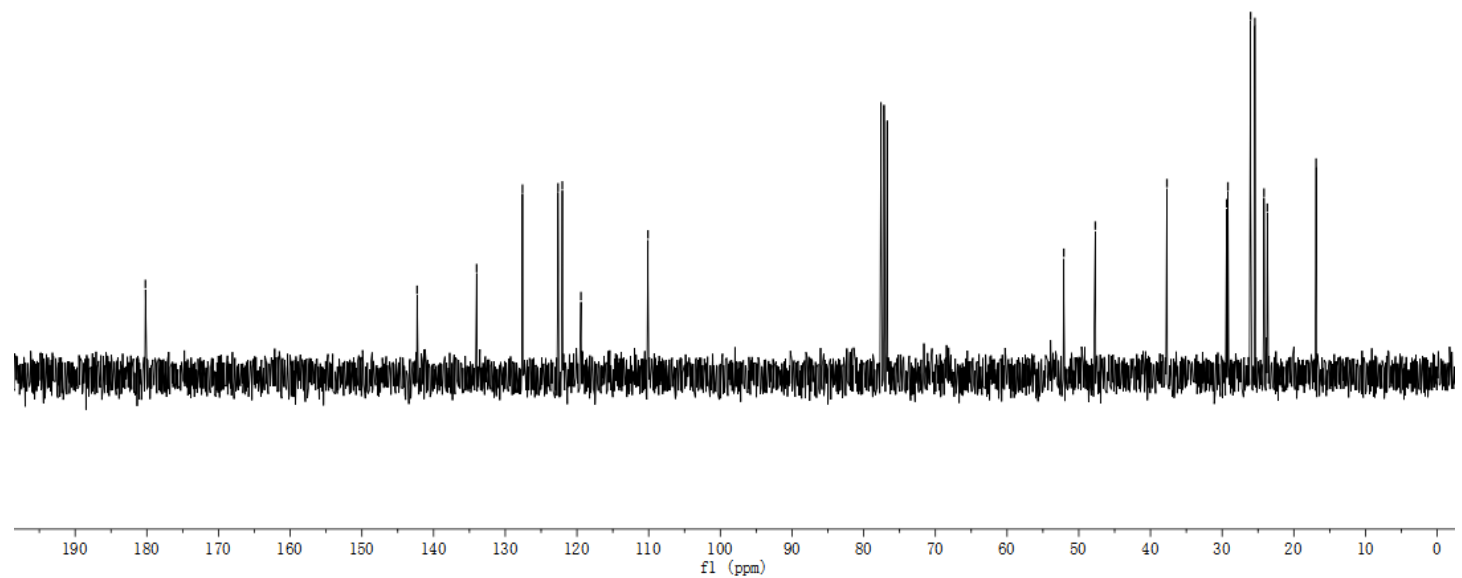
${ }^{1} \mathbf{H}$ NMR of compound $3 \mathbf{f}\left(300 \mathrm{MHz}\right.$ in $\left.\mathrm{CDCl}_{3}\right)$

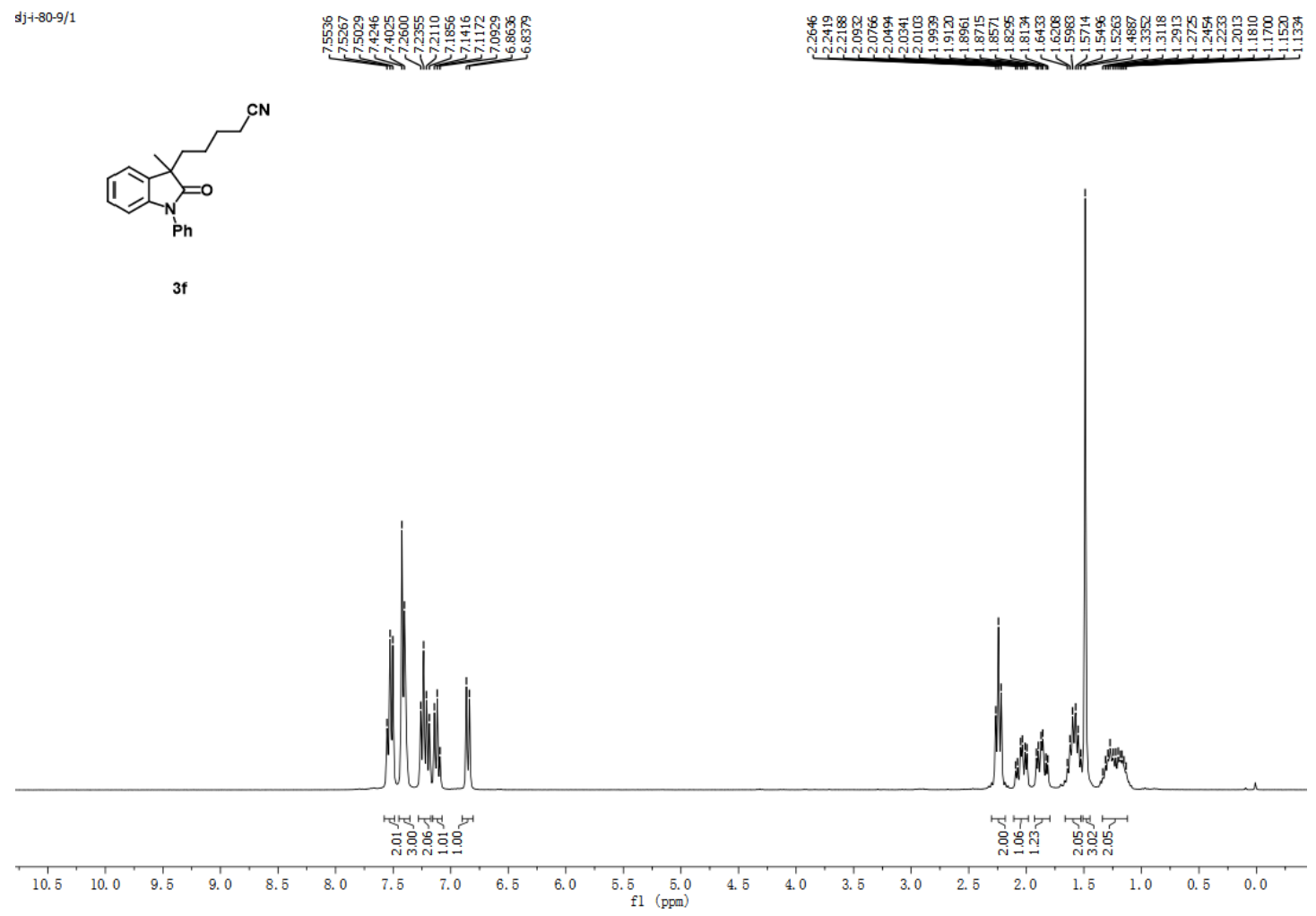

${ }^{13} \mathrm{C}\left\{{ }^{1} \mathrm{H}\right\}$ NMR of compound $3 \mathrm{f}\left(75 \mathrm{MHz}\right.$ in $\left.\mathrm{CDCl}_{3}\right)$

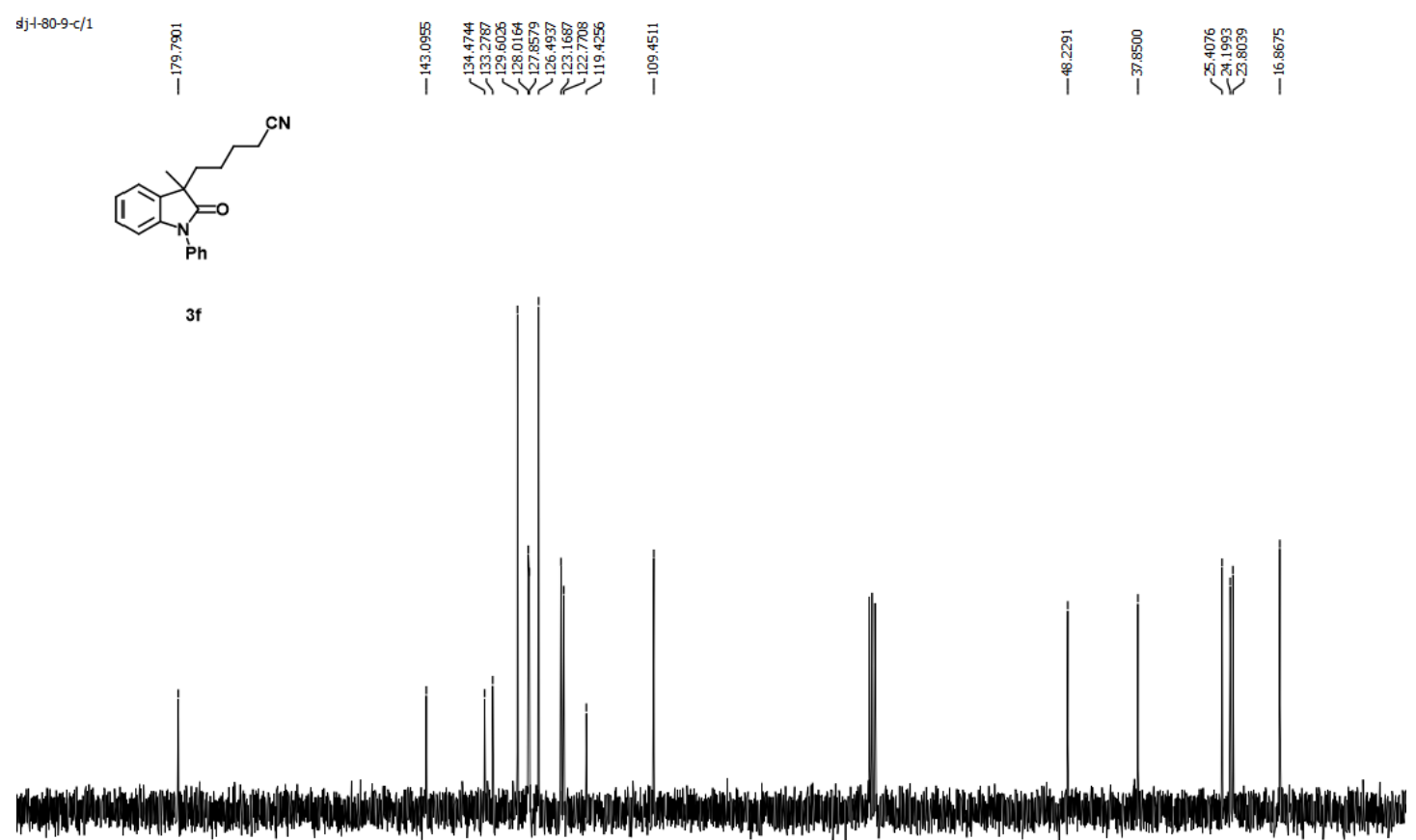

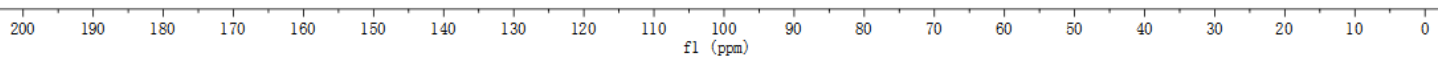


${ }^{1} \mathbf{H}$ NMR of compound $\mathbf{3 g}$ (300 MHz in $\mathrm{CDCl}_{3}$ )

sjj-80-11/1

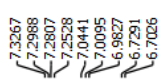

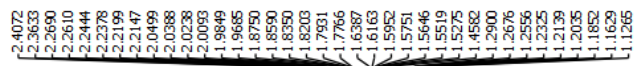

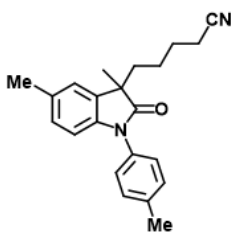

$3 \mathrm{~g}$

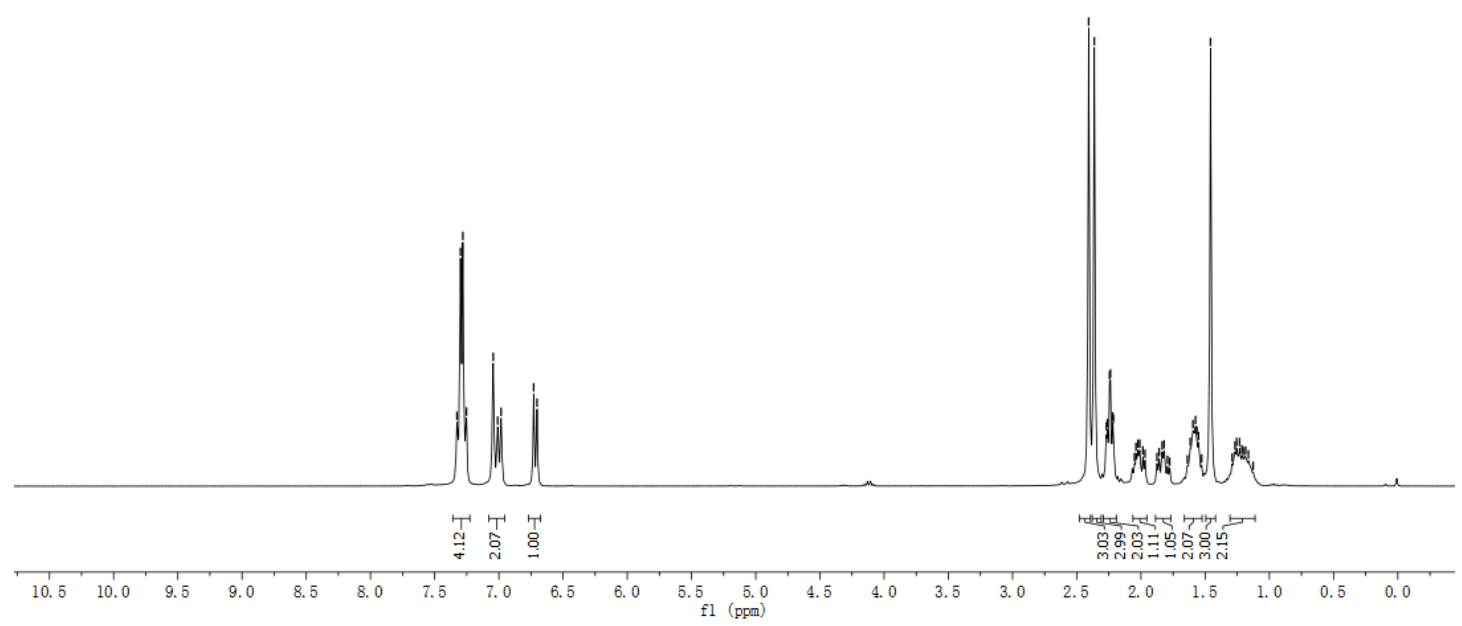

${ }^{13} \mathrm{C}\left\{{ }^{1} \mathrm{H}\right\}$ NMR of compound $\mathbf{3 g}$ (75 MHz in $\mathrm{CDCl}_{3}$ )

djj--80-11-c/ 1

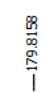<smiles>Cc1ccc(N2C(=O)C(C)(CCCCC#N)c3cc(C)ccc32)cc1</smiles>

$3 g$

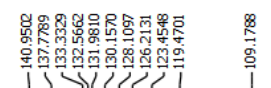

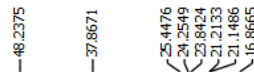

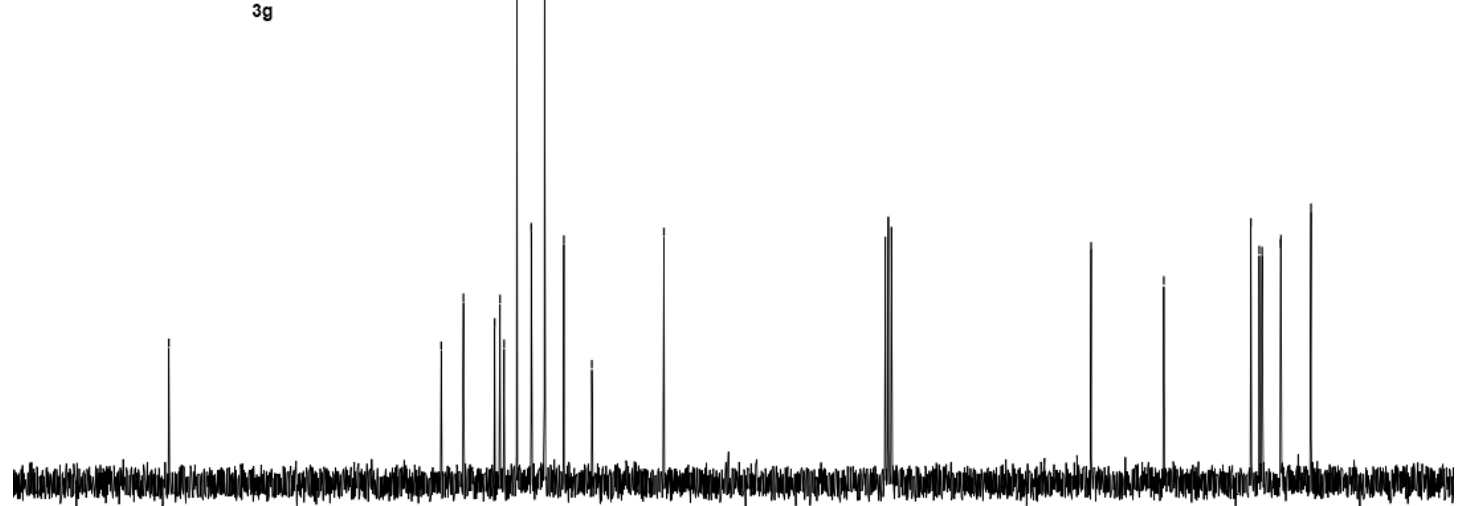

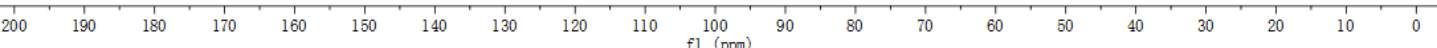


${ }^{1} \mathbf{H}$ NMR of compound $\mathbf{3 h}\left(300 \mathrm{MHz}\right.$ in $\left.\mathrm{CDCl}_{3}\right)$

sji-80-12/1

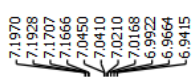

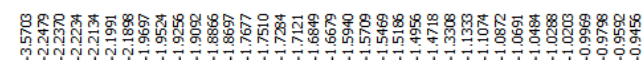

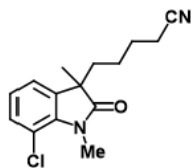

$3 \mathrm{~h}$

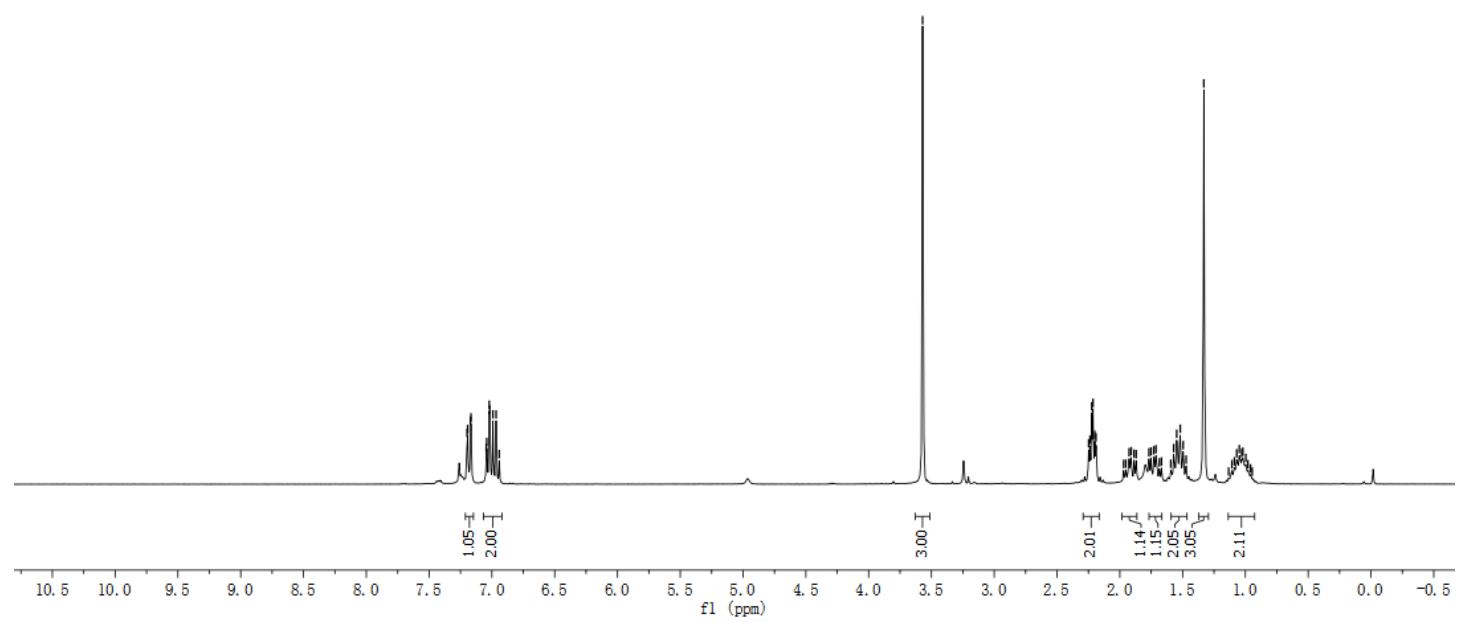

${ }^{13} \mathrm{C}\left\{{ }^{1} \mathrm{H}\right\}$ NMR of compound $3 \mathrm{~h}\left(75 \mathrm{MHz}\right.$ in $\left.\mathrm{CDCl}_{3}\right)$

djj-80-12-c/1

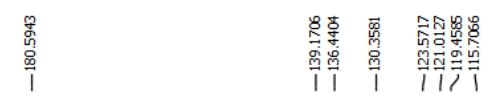

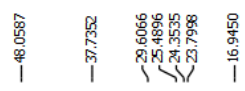

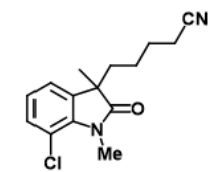

$3 \mathrm{~h}$

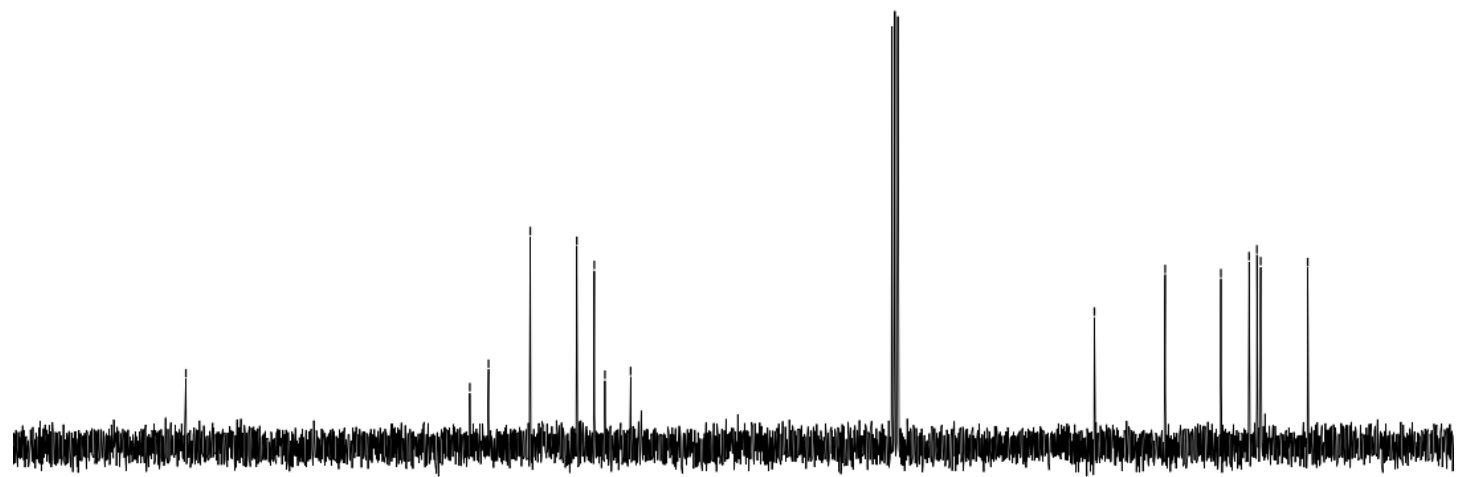

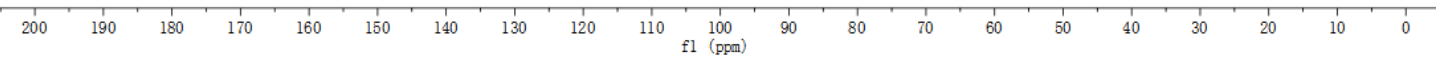


${ }^{1} \mathbf{H}$ NMR of compound $\mathbf{3 i}\left(300 \mathrm{MHz}\right.$ in $\left.\mathrm{CDCl}_{3}\right)$

s.) -- $-89-3 / 1$

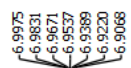

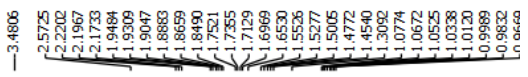

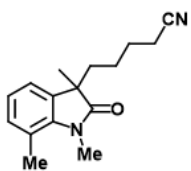

$3 i$

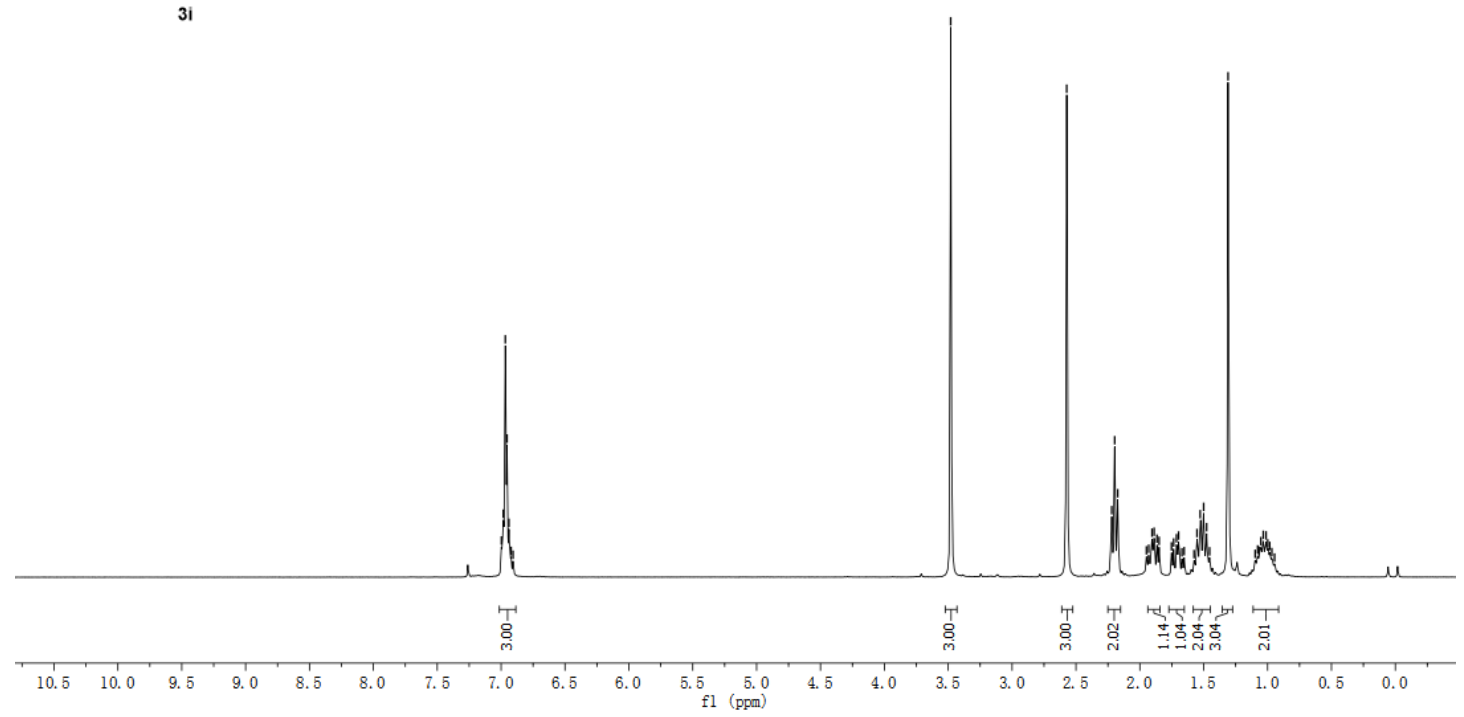

${ }^{13} \mathrm{C}\left\{{ }^{1} \mathrm{H}\right\}$ NMR of compound $3 \mathbf{i}\left(75 \mathrm{MHz}\right.$ in $\mathrm{CDCl}_{3}$ )

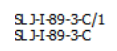

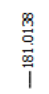

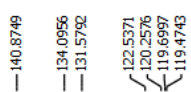

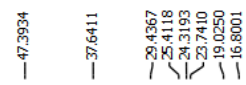

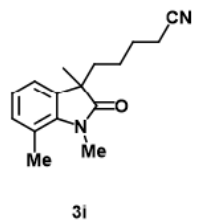

31

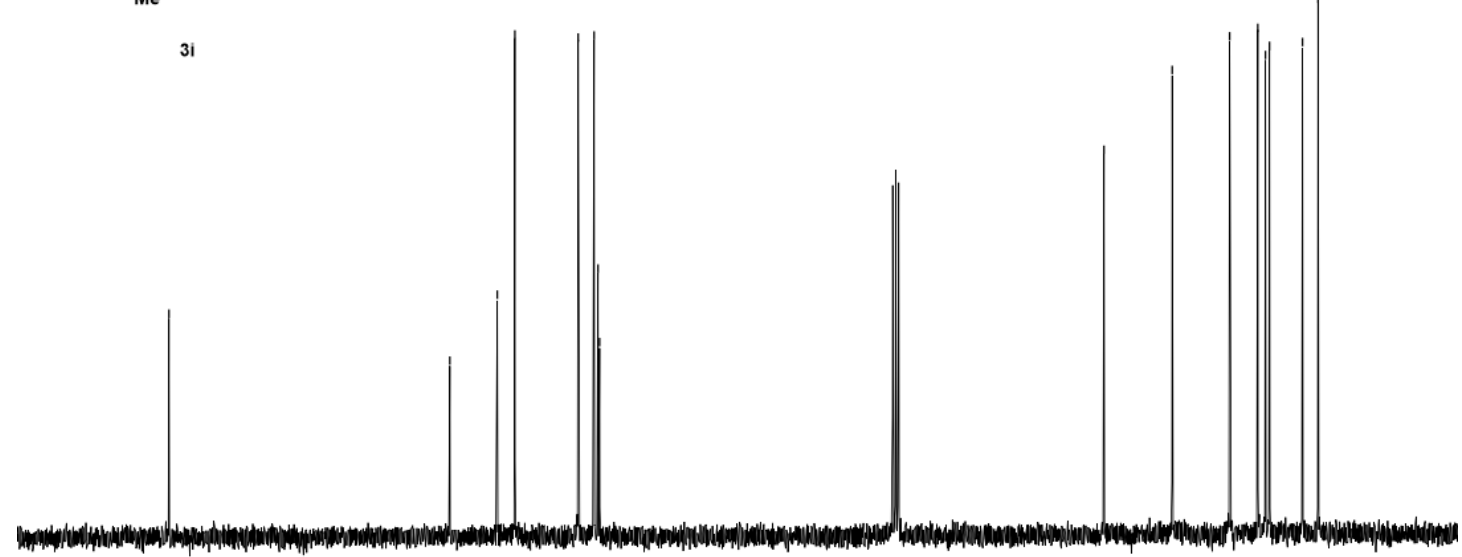

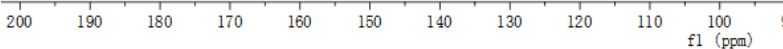


${ }^{1} \mathbf{H}$ NMR of compound $\mathbf{3 j}\left(300 \mathrm{MHz}\right.$ in $\mathrm{CDCl}_{3}$ )

s. $-[-193-1 / 1$

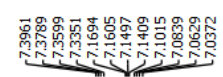

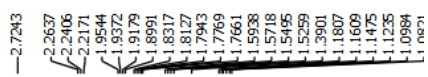

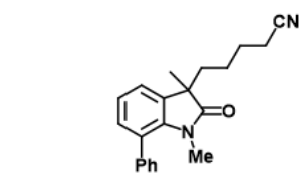

3j

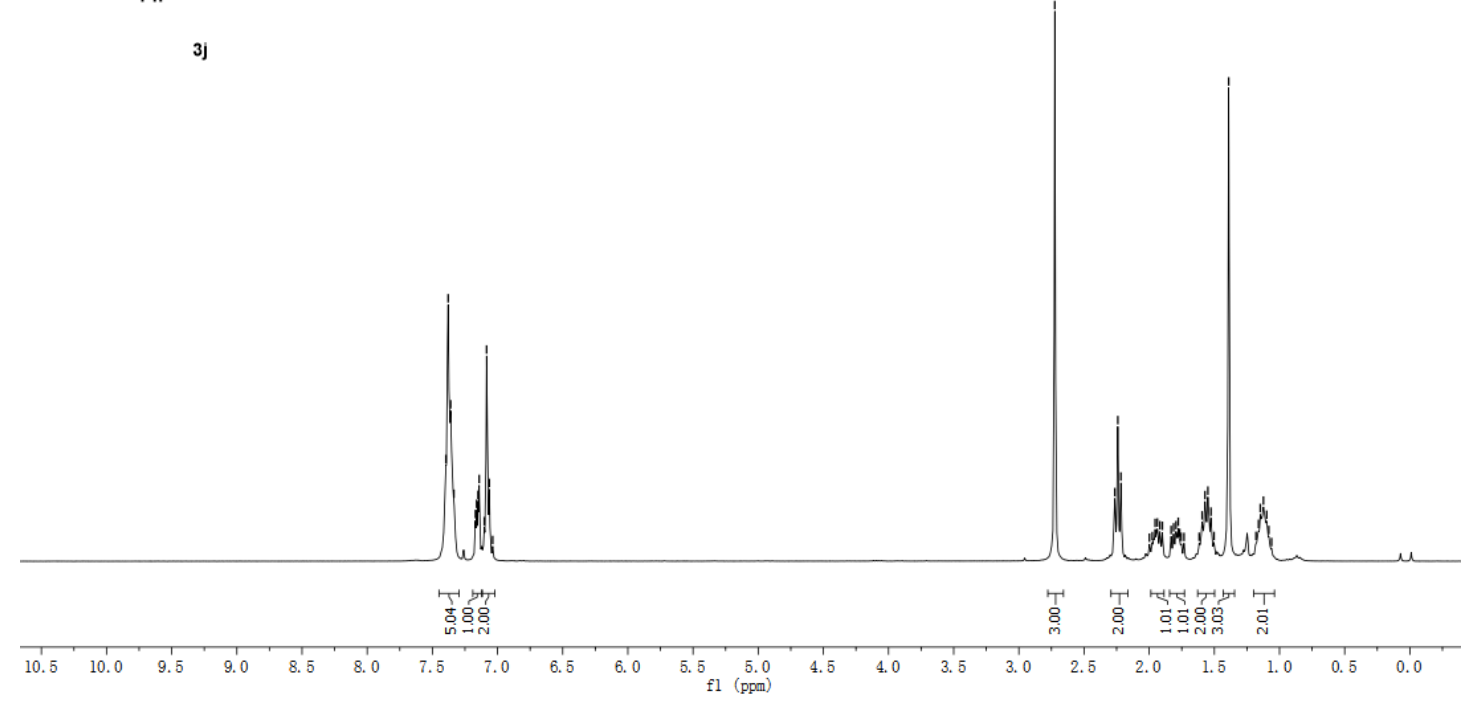

${ }^{13} \mathbf{C}\left\{{ }^{1} \mathrm{H}\right\}$ NMR of compound $\mathbf{3} \mathbf{j}\left(75 \mathrm{MHz}\right.$ in $\mathrm{CDCl}_{3}$ )

s. $-1-193-1-C / 2$

\begin{tabular}{l}
$\frac{8}{0}$ \\
$\frac{0}{0}$ \\
$\frac{0}{1}$ \\
\hline
\end{tabular}

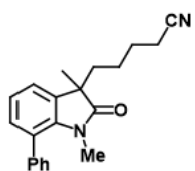

3j

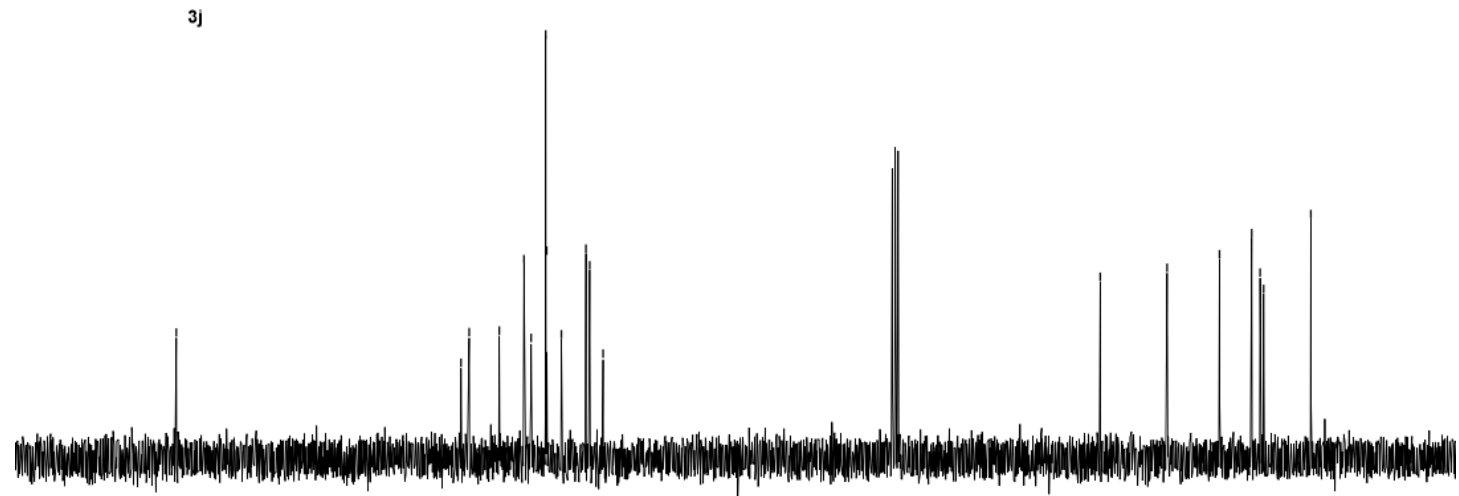

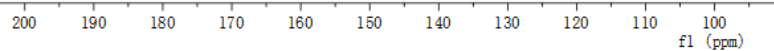


${ }^{\mathbf{1}} \mathbf{H} \mathbf{N M R}$ of compound $\mathbf{3 k}$ and $\mathbf{3 k}$ ' (300 $\mathrm{MHz}$ in $\mathrm{CDCl}_{3}$ )

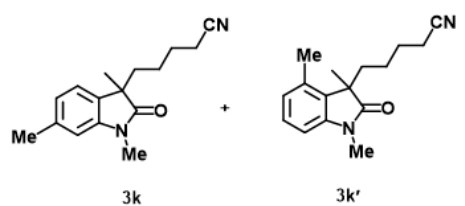

$3 k$

$3 \mathrm{k}^{\prime}$

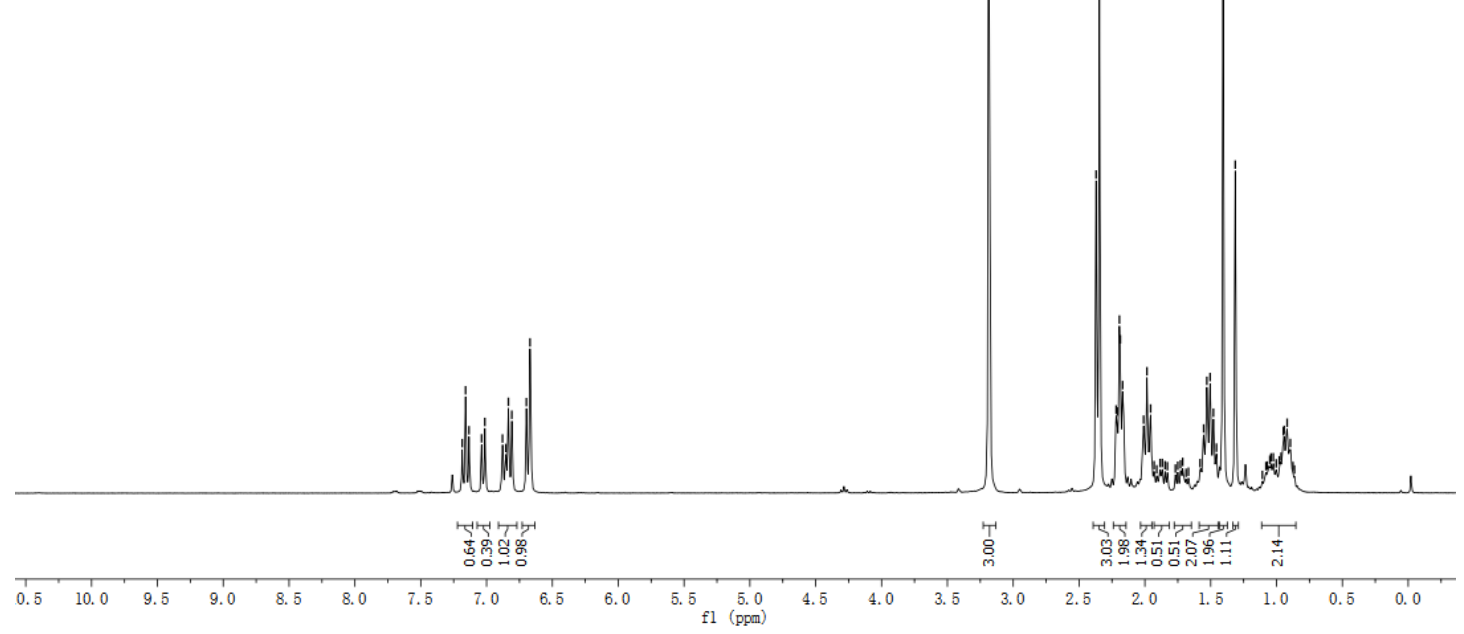

${ }^{13} \mathbf{C}\left\{{ }^{1} \mathbf{H}\right\}$ NMR of compound $\mathbf{3 k}$ and $\mathbf{3 k}$ ' (75 $\mathrm{MHz}$ in $\mathrm{CDCl}_{3}$ )

sj--1-80-4-c/1
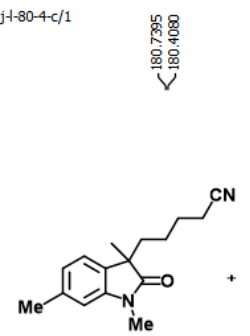

$3 k$

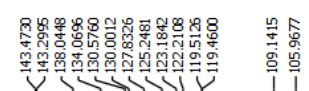<smiles>Cc1cccc2c1C(C)(CCCCC#N)C(=O)N2C</smiles>

$3 \mathbf{k}^{\prime}$

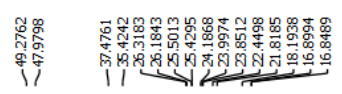

年

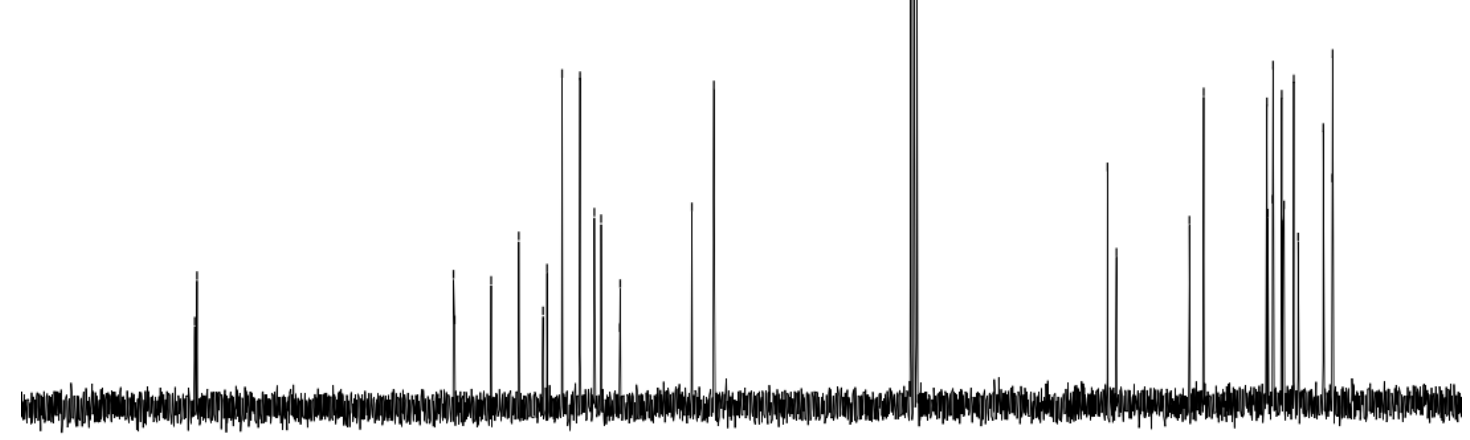

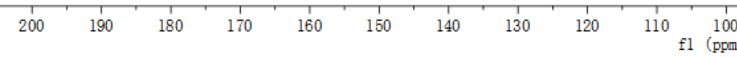


${ }^{\mathbf{1}} \mathbf{H}$ NMR of compound $\mathbf{3 I}\left(300 \mathrm{MHz}\right.$ in $\mathrm{CDCl}_{3}$ )

จ. $\mathrm{J}-\mathrm{I}-80-2 / 1$

31

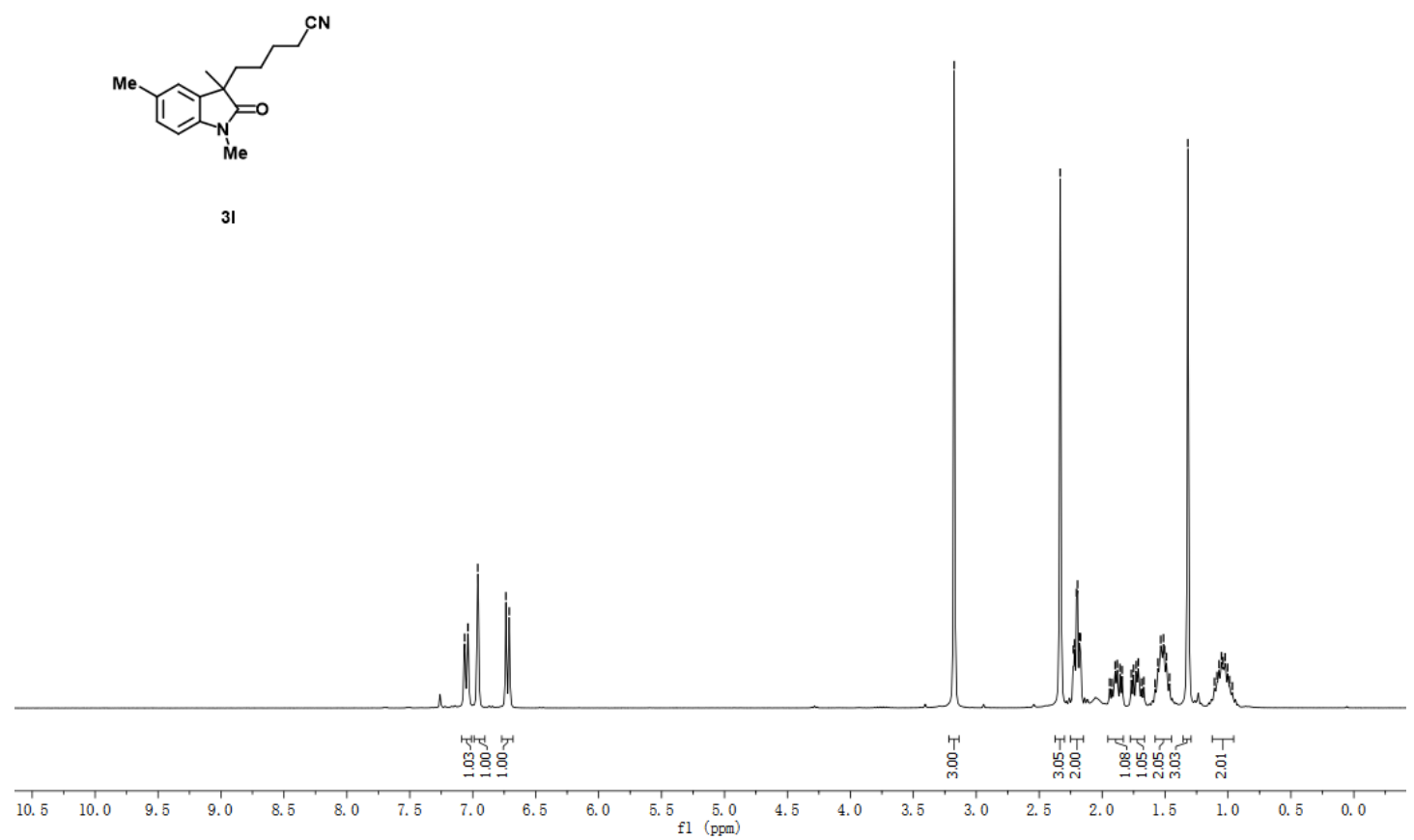

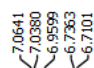

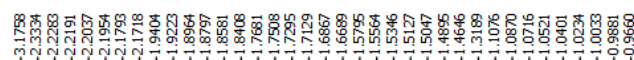

${ }^{13} \mathrm{C}\left\{{ }^{1} \mathrm{H}\right\}$ NMR of compound $3 \mathbf{3}\left(75 \mathrm{MHz}\right.$ in $\mathrm{CDCl}_{3}$ )

$\$ j-1-80-2-c / 1$

愈

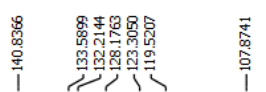

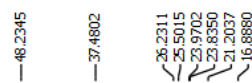

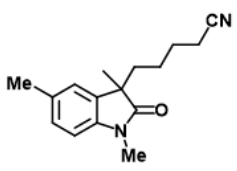

3)

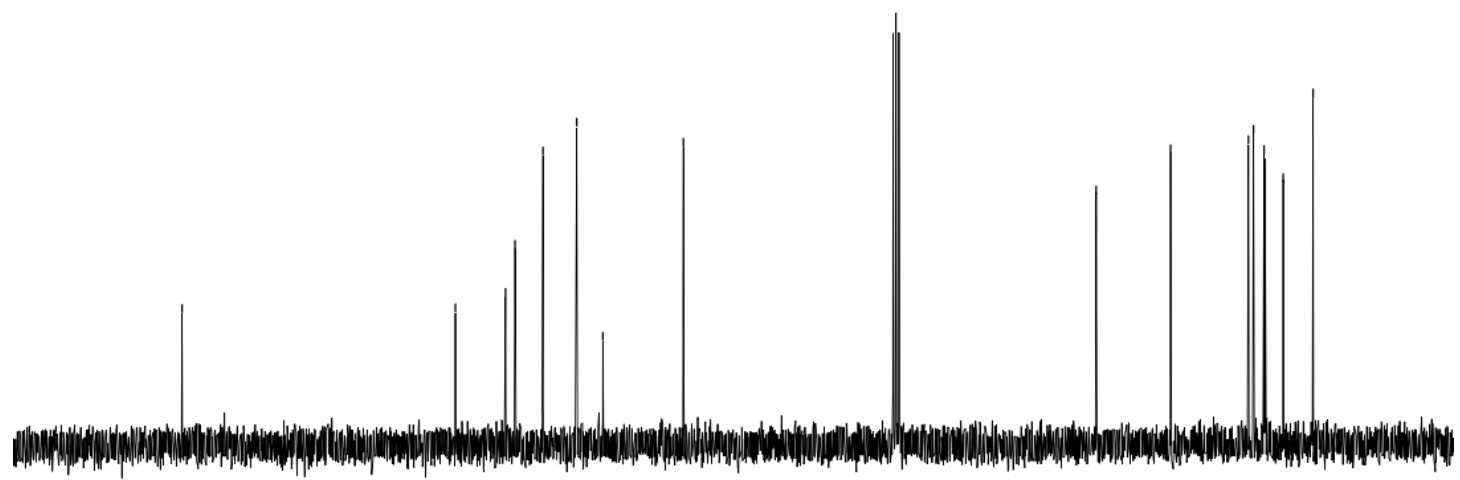

$170 \quad 160$

$150 \quad 140$

$130 \quad 120$

$10 \stackrel{100}{\text { fl }(\mathrm{ppm})}$ 
${ }^{1} \mathbf{H}$ NMR of compound $3 \mathrm{~m}\left(300 \mathrm{MHz}\right.$ in $\mathrm{CDCl}_{3}$ )

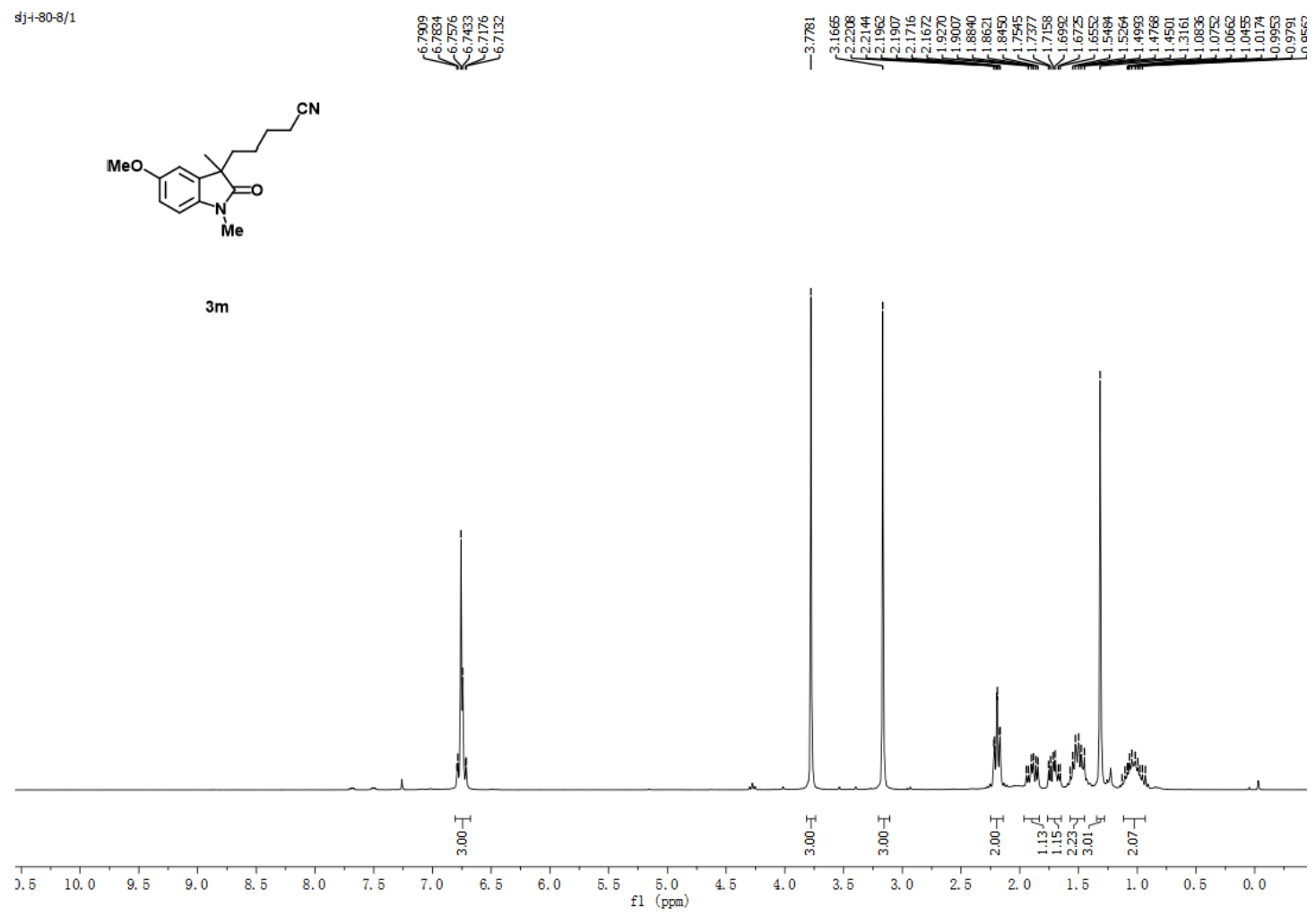

${ }^{13} \mathrm{C}\left\{{ }^{1} \mathrm{H}\right\}$ NMR of compound $3 \mathrm{~m}\left(75 \mathrm{MHz}\right.$ in $\left.\mathrm{CDCl}_{3}\right)$

sjj-80-8-c/1

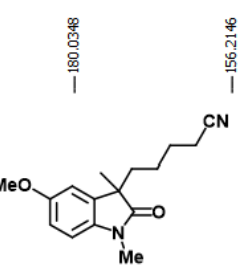

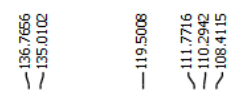

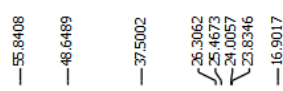

$3 m$

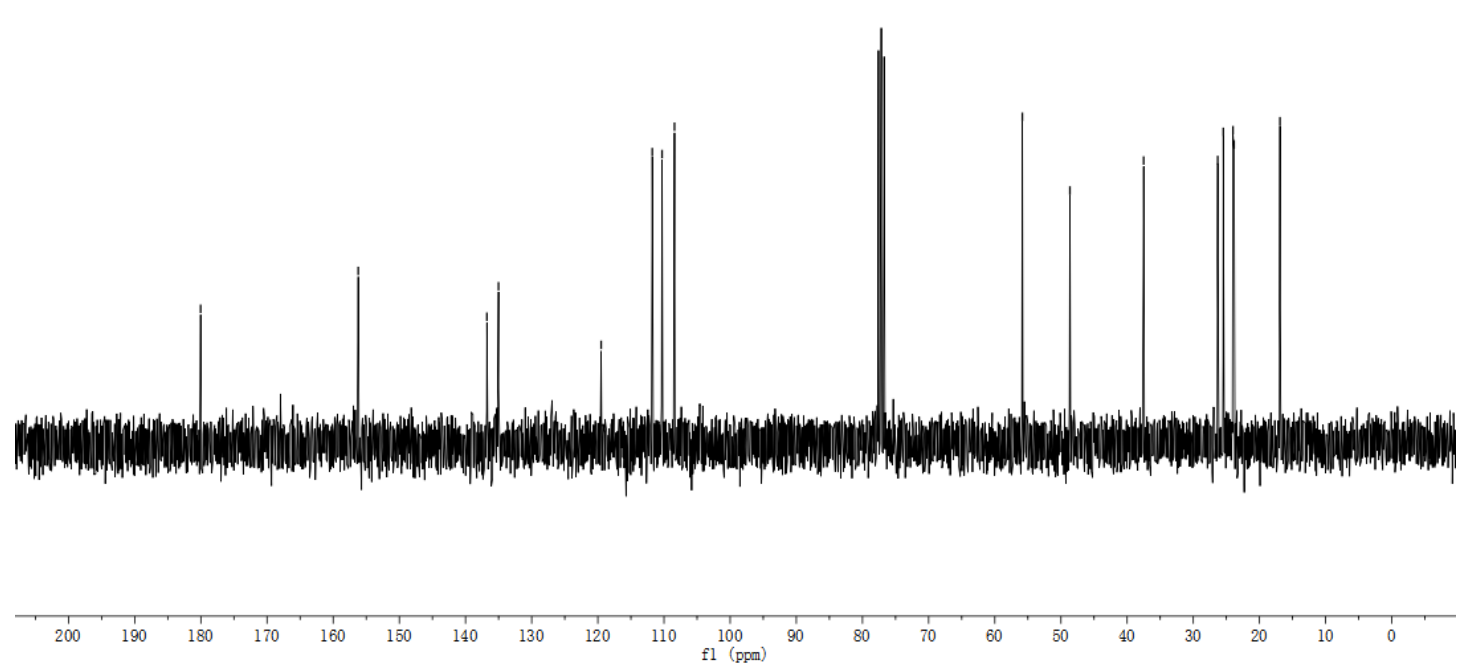


${ }^{1} \mathbf{H}$ NMR of compound $\mathbf{3 n}\left(300 \mathrm{MHz}\right.$ in $\left.\mathrm{CDCl}_{3}\right)$

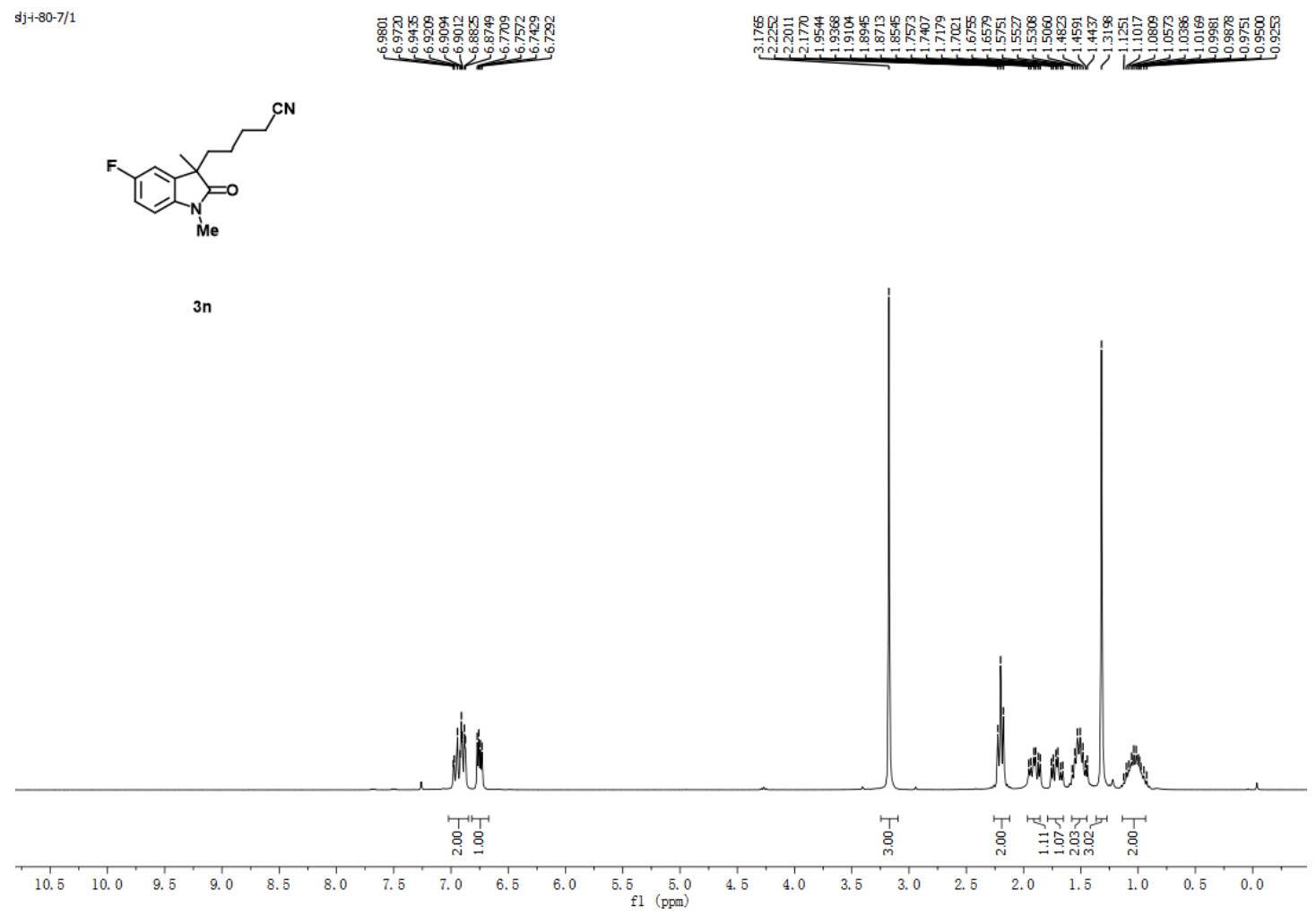

${ }^{13} \mathrm{C}\left\{{ }^{1} \mathrm{H}\right\}$ NMR of compound $3 \mathrm{n}\left(75 \mathrm{MHz}\right.$ in $\left.\mathrm{CDCl}_{3}\right)$

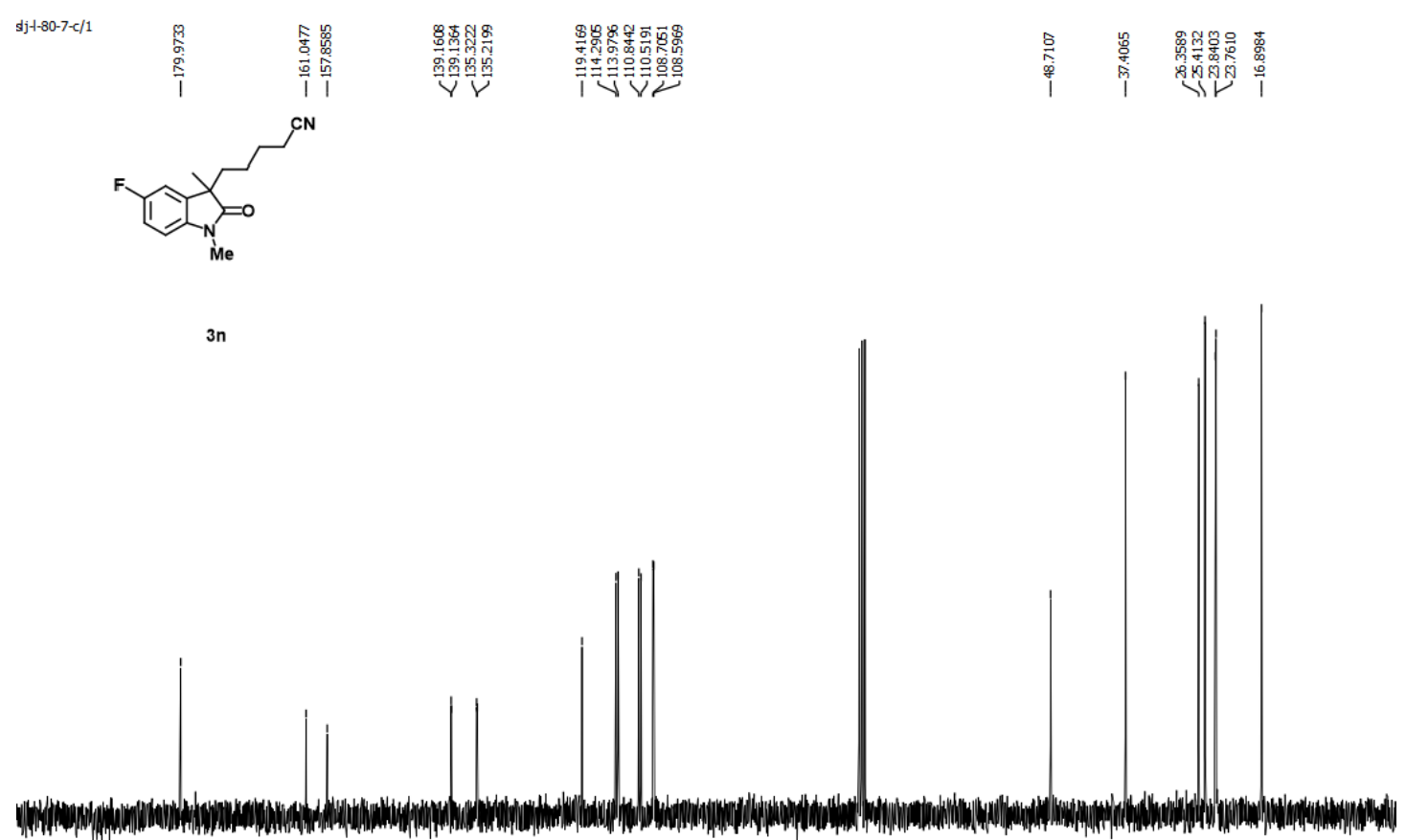

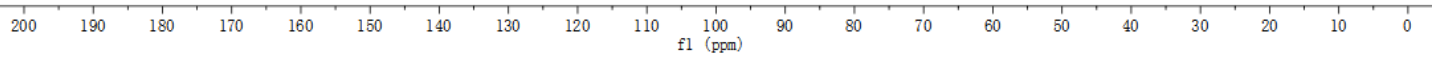


${ }^{19} \mathrm{~F} \mathrm{NMR}$ of compound $3 \mathrm{n}\left(282 \mathrm{MHz}\right.$ in $\left.\mathrm{CDCl}_{3}\right)$ jj $j-80-7 F / 1$

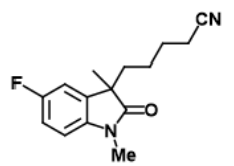

3n

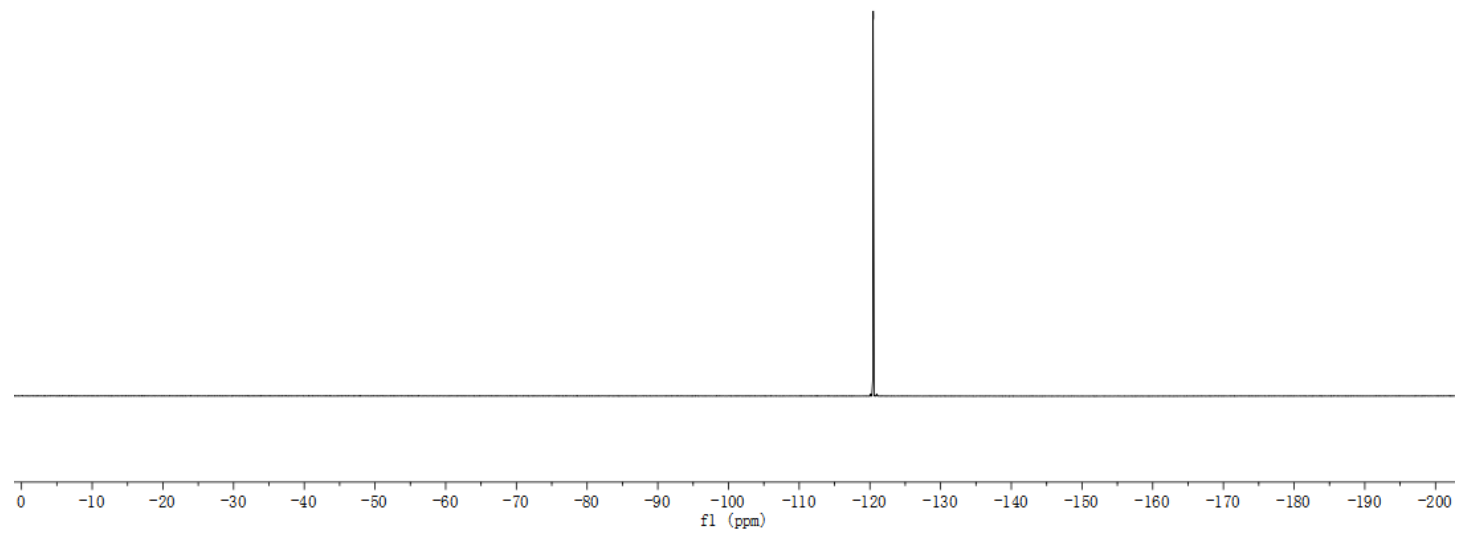

${ }^{1} \mathbf{H}$ NMR of compound $30\left(300 \mathrm{MHz}\right.$ in $\left.\mathrm{CDCl}_{3}\right)$

sjj-80-6/1

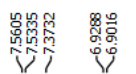
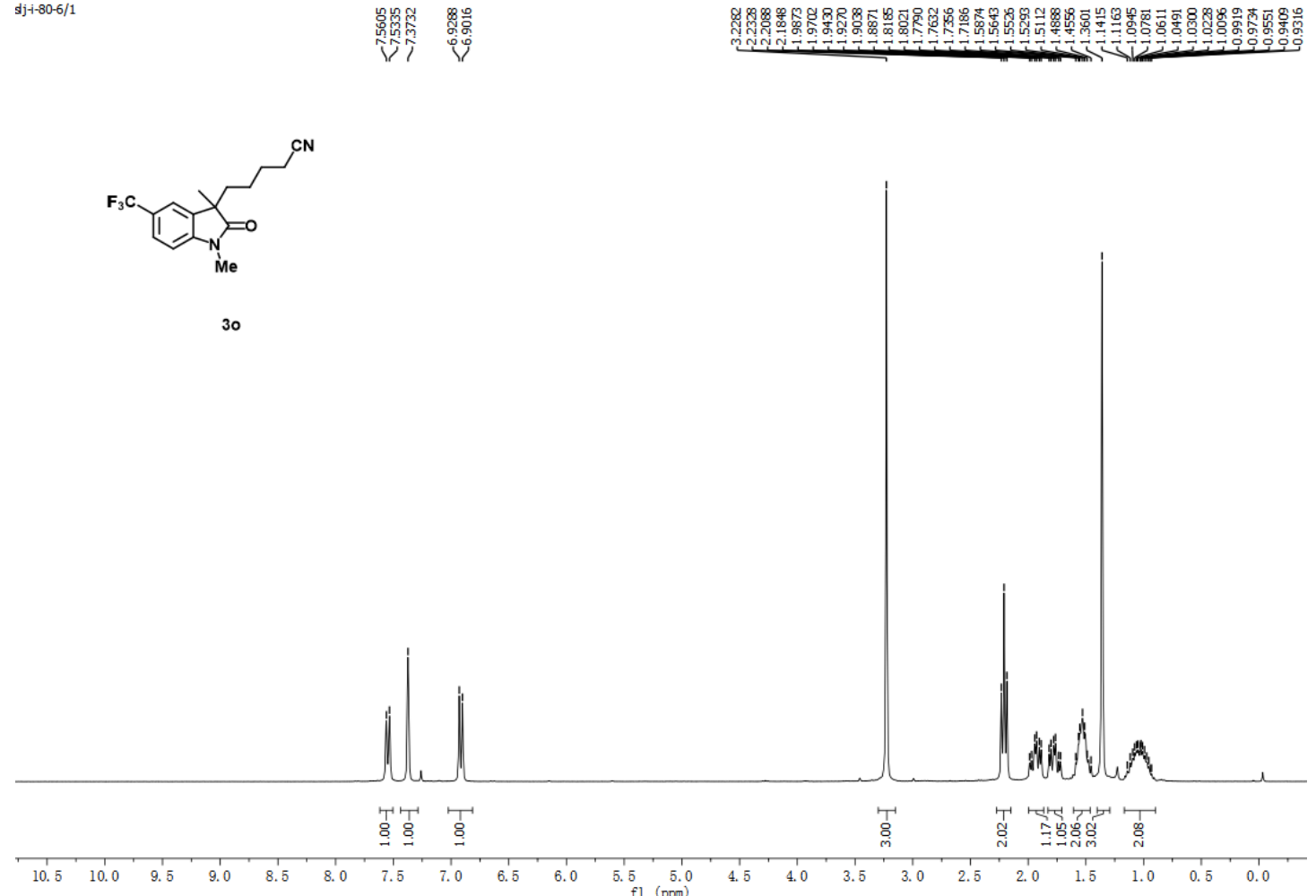

30 
${ }^{13} \mathrm{C}\left\{{ }^{1} \mathrm{H}\right\}$ NMR of compound $30\left(75 \mathrm{MHz}\right.$ in $\left.\mathrm{CDCl}_{3}\right)$

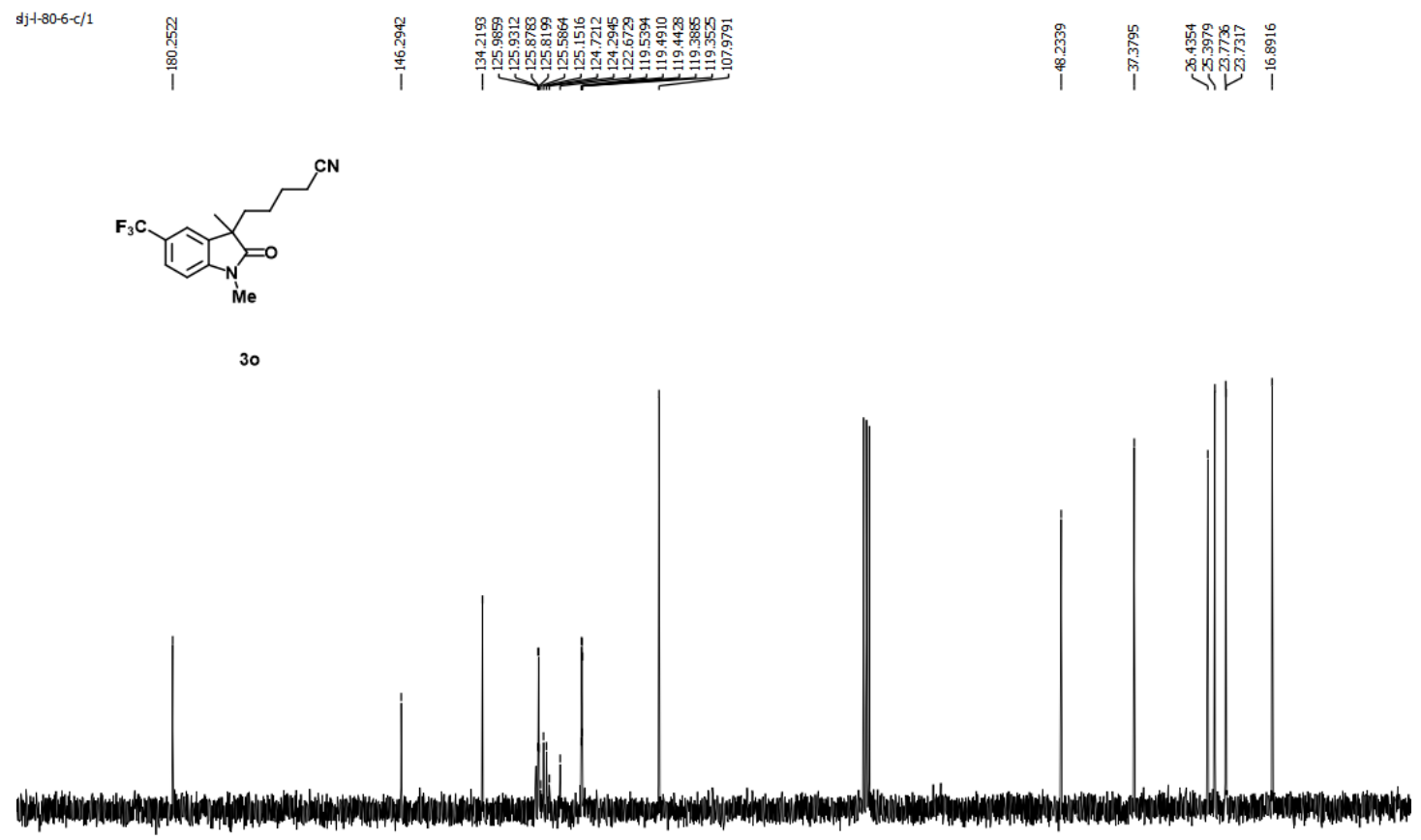

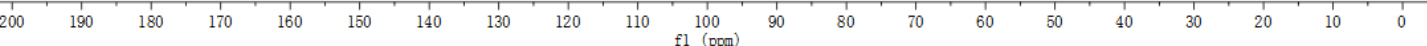

${ }^{19} \mathrm{~F}$ NMR of compound $30\left(282 \mathrm{MHz}\right.$ in $\left.\mathrm{CDCl}_{3}\right)$

sj $-1-80-6-F / 1$

畩

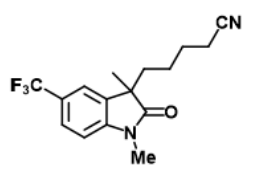

30

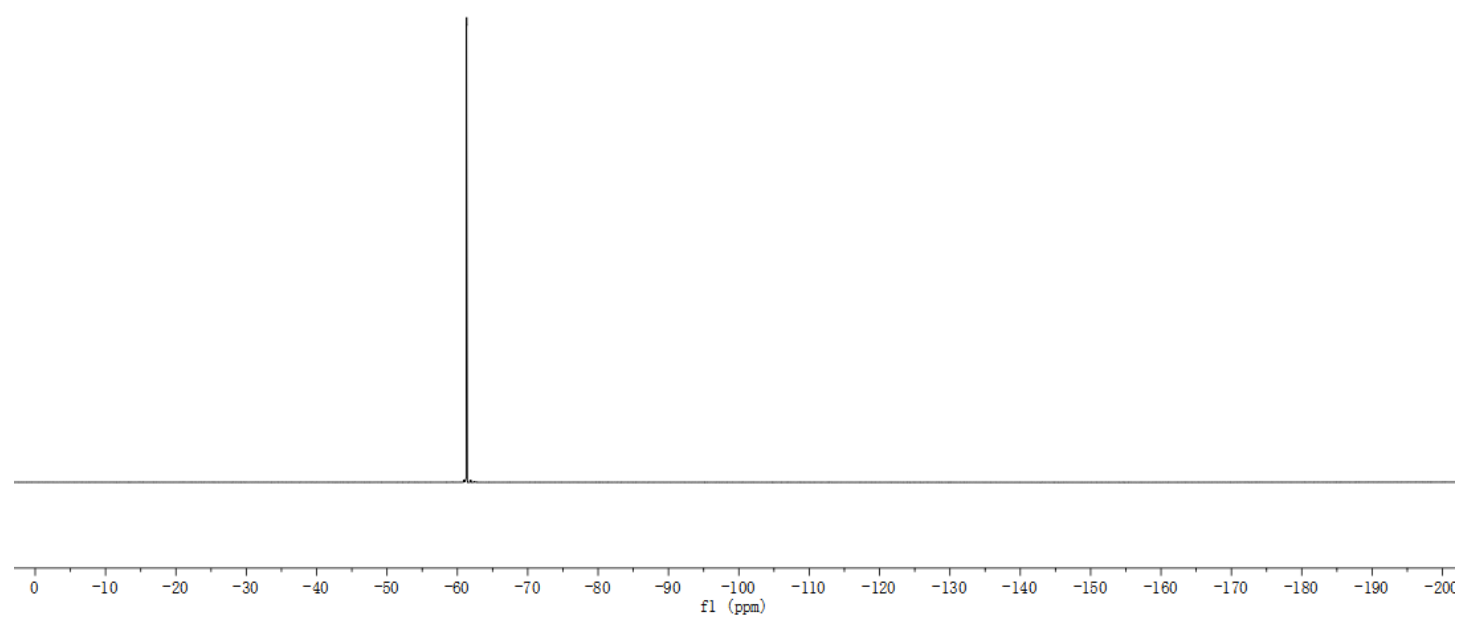


${ }^{1} \mathbf{H}$ NMR of compound $3 p\left(300 \mathrm{MHz}\right.$ in $\left.\mathrm{CDCl}_{3}\right)$

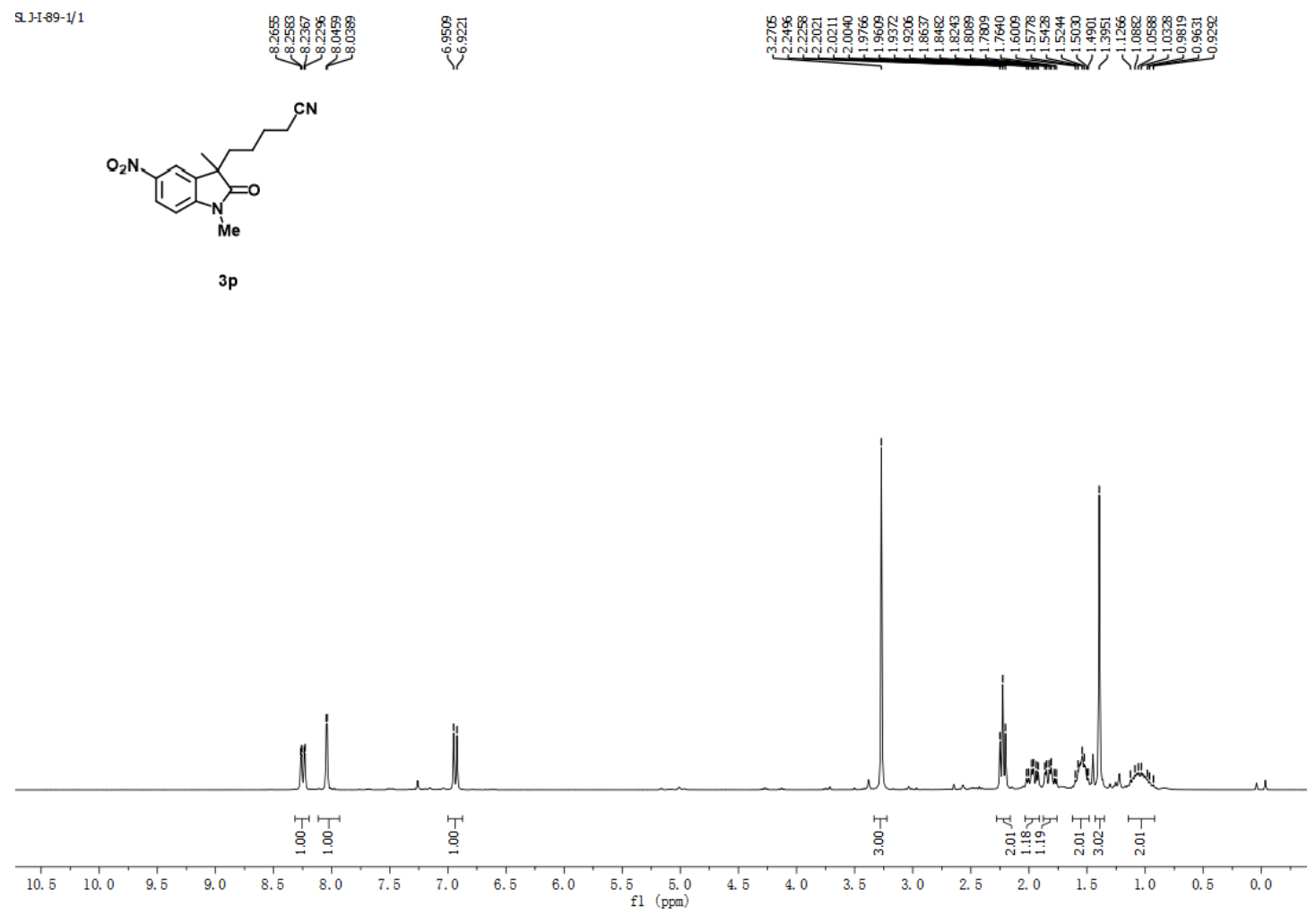

${ }^{13} \mathrm{C}\left\{{ }^{1} \mathrm{H}\right\}$ NMR of compound $3 p\left(75 \mathrm{MHz}\right.$ in $\left.\mathrm{CDCl}_{3}\right)$

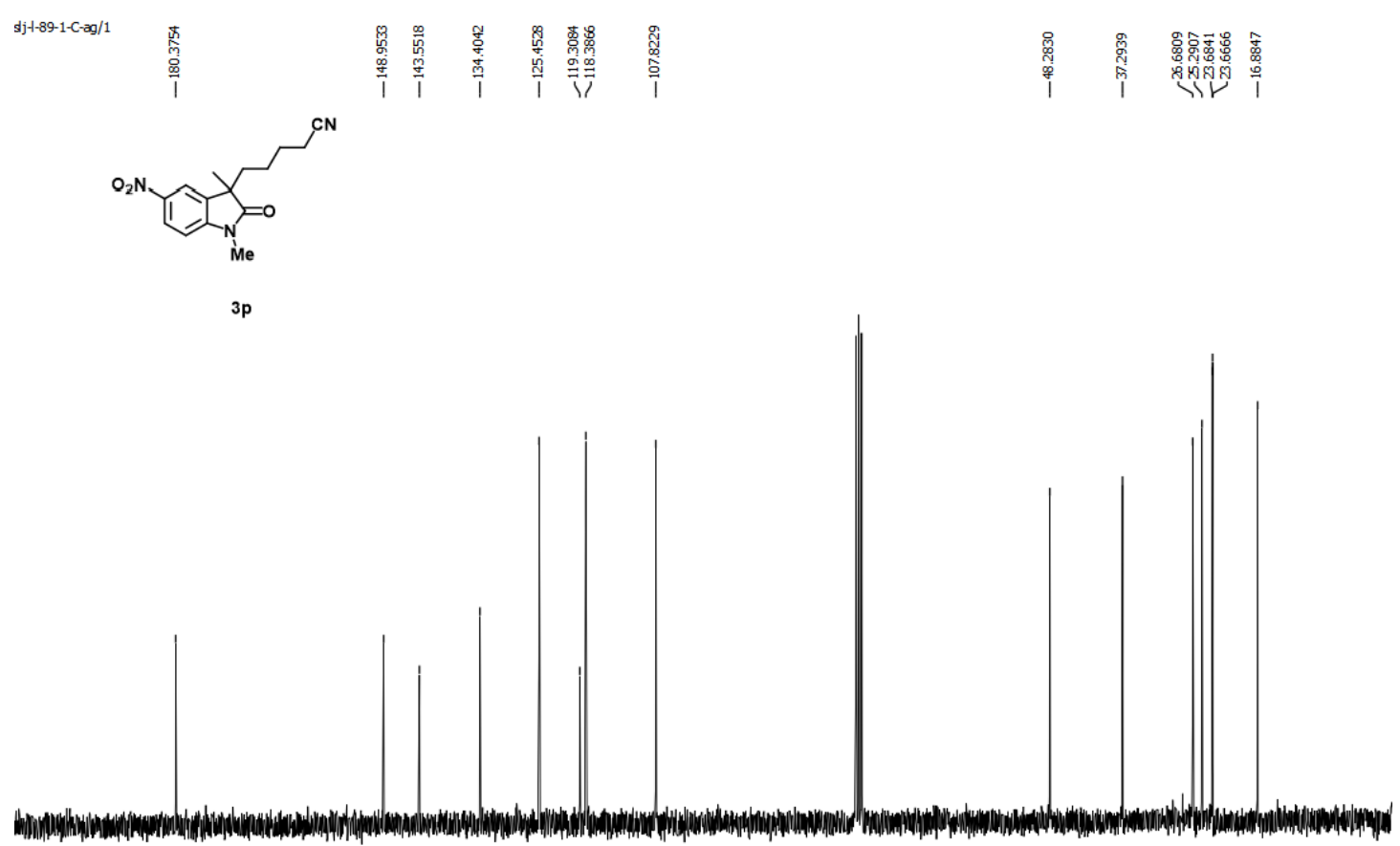

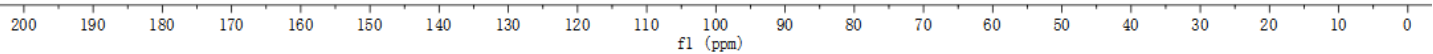


${ }^{\mathbf{1}} \mathbf{H} \mathbf{N M R}$ of compound $\mathbf{3 q}$ and $\mathbf{3 q} \mathbf{q}^{\prime}\left(300 \mathrm{MHz}\right.$ in $\mathrm{CDCl}_{3}$ )

s.]-I-1-121-3/1

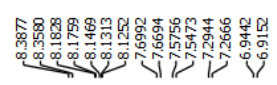

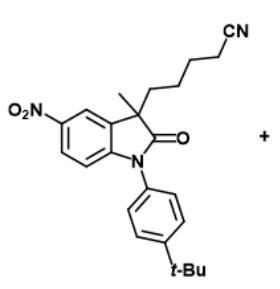

$3 q$

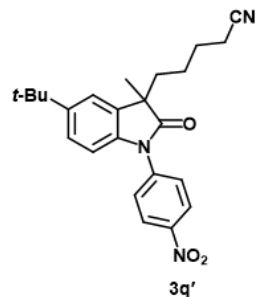

$3 q^{\prime}$

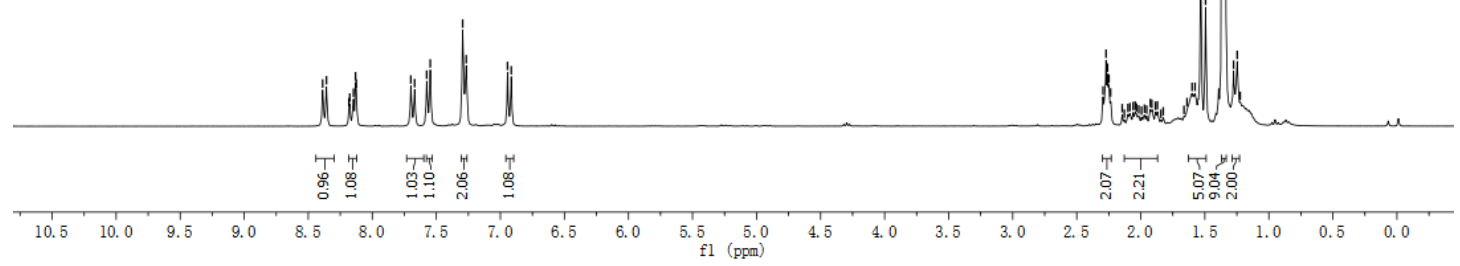

${ }^{13} \mathbf{C}\left\{{ }^{1} \mathrm{H}\right\}$ NMR of compound $\mathbf{3 q}$ and $\mathbf{3} \mathbf{q}^{\mathbf{\prime}}\left(75 \mathrm{MHz}\right.$ in $\mathrm{CDCl}_{3}$ )

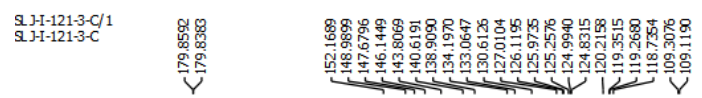

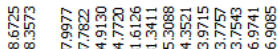

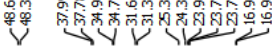

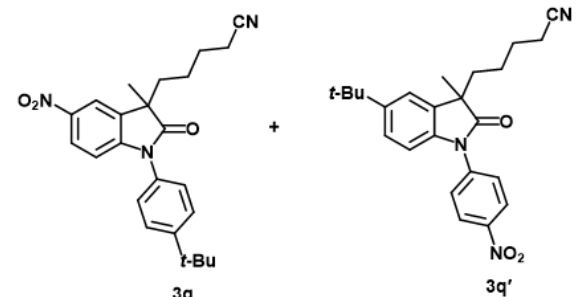

39

3q'

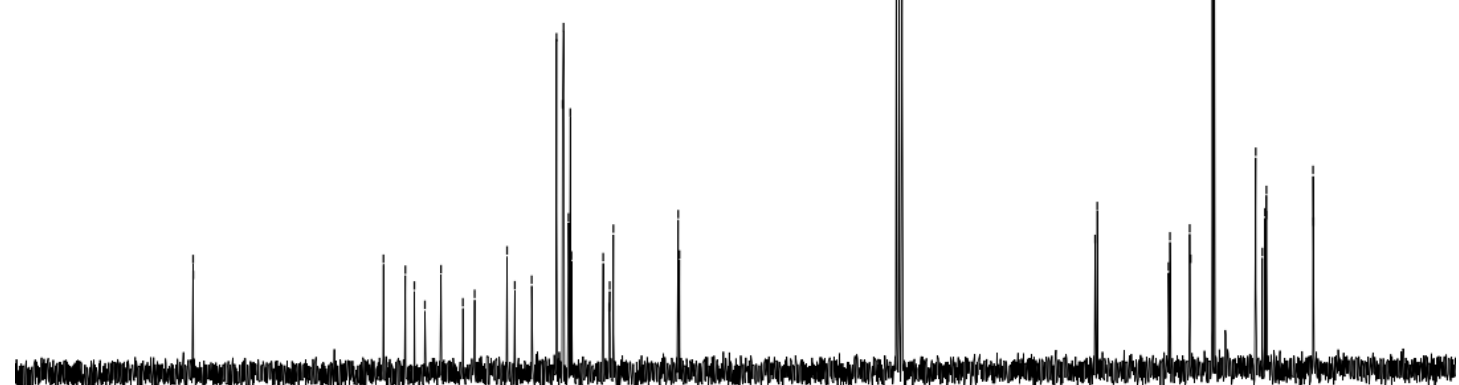

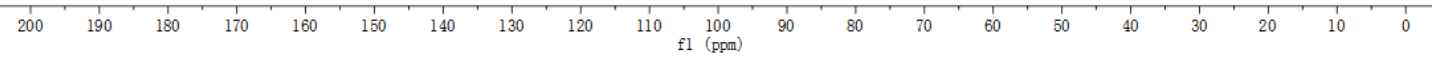


${ }^{1} \mathbf{H}$ NMR of compound $3 \mathbf{r}\left(300 \mathrm{MHz}\right.$ in $\left.\mathrm{CDCl}_{3}\right)$

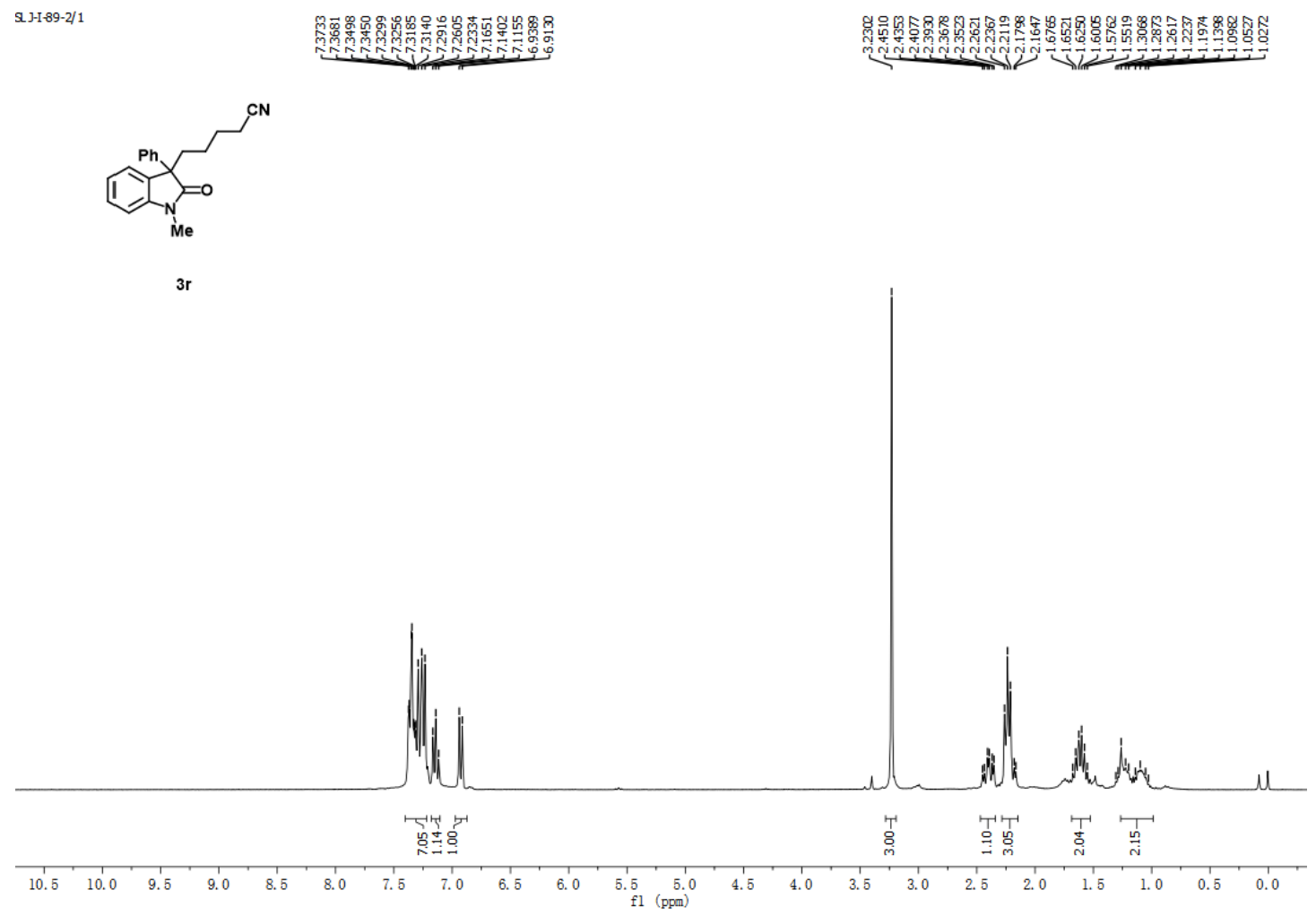

${ }^{13} \mathrm{C}\left\{{ }^{1} \mathrm{H}\right\}$ NMR of compound $3 r\left(75 \mathrm{MHz}\right.$ in $\left.\mathrm{CDCl}_{3}\right)$

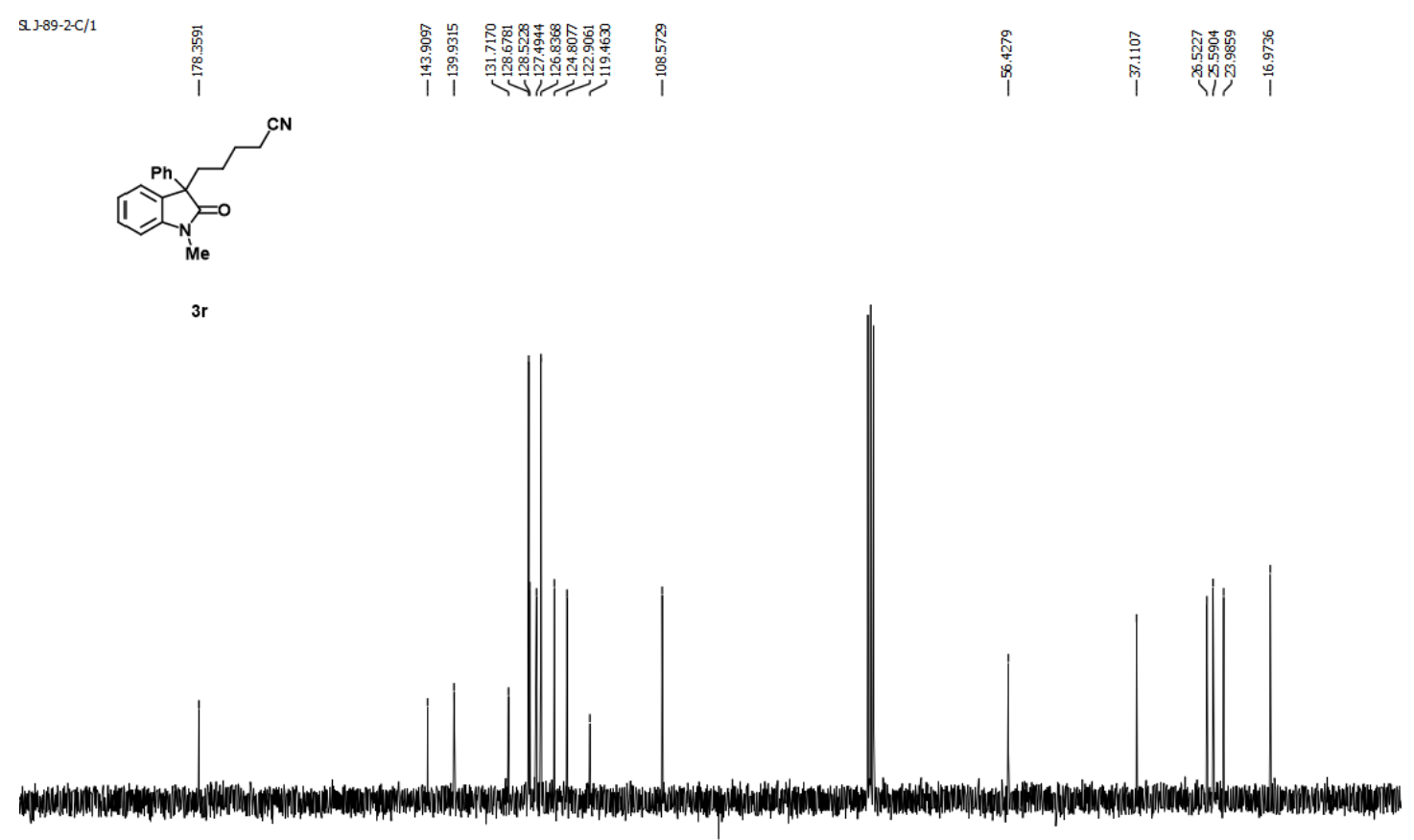

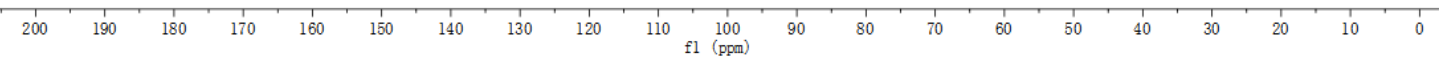


${ }^{1} \mathbf{H}$ NMR of compound $3 \mathbf{s}\left(300 \mathrm{MHz}\right.$ in $\left.\mathrm{CDCl}_{3}\right)$

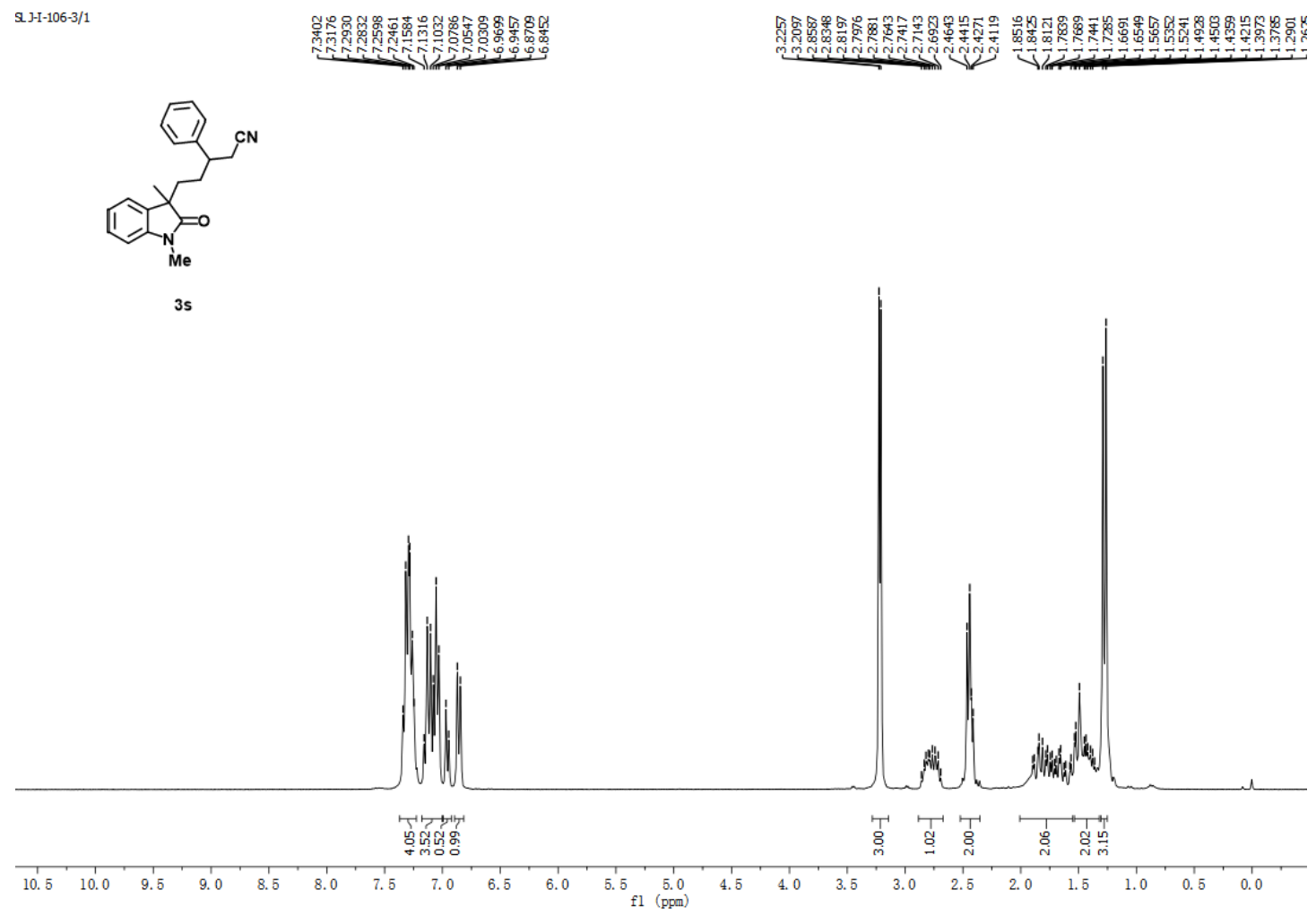

${ }^{13} \mathrm{C}\left\{{ }^{1} \mathrm{H}\right\}$ NMR of compound 3s $\left(75 \mathrm{MHz}\right.$ in $\left.\mathrm{CDCl}_{3}\right)$

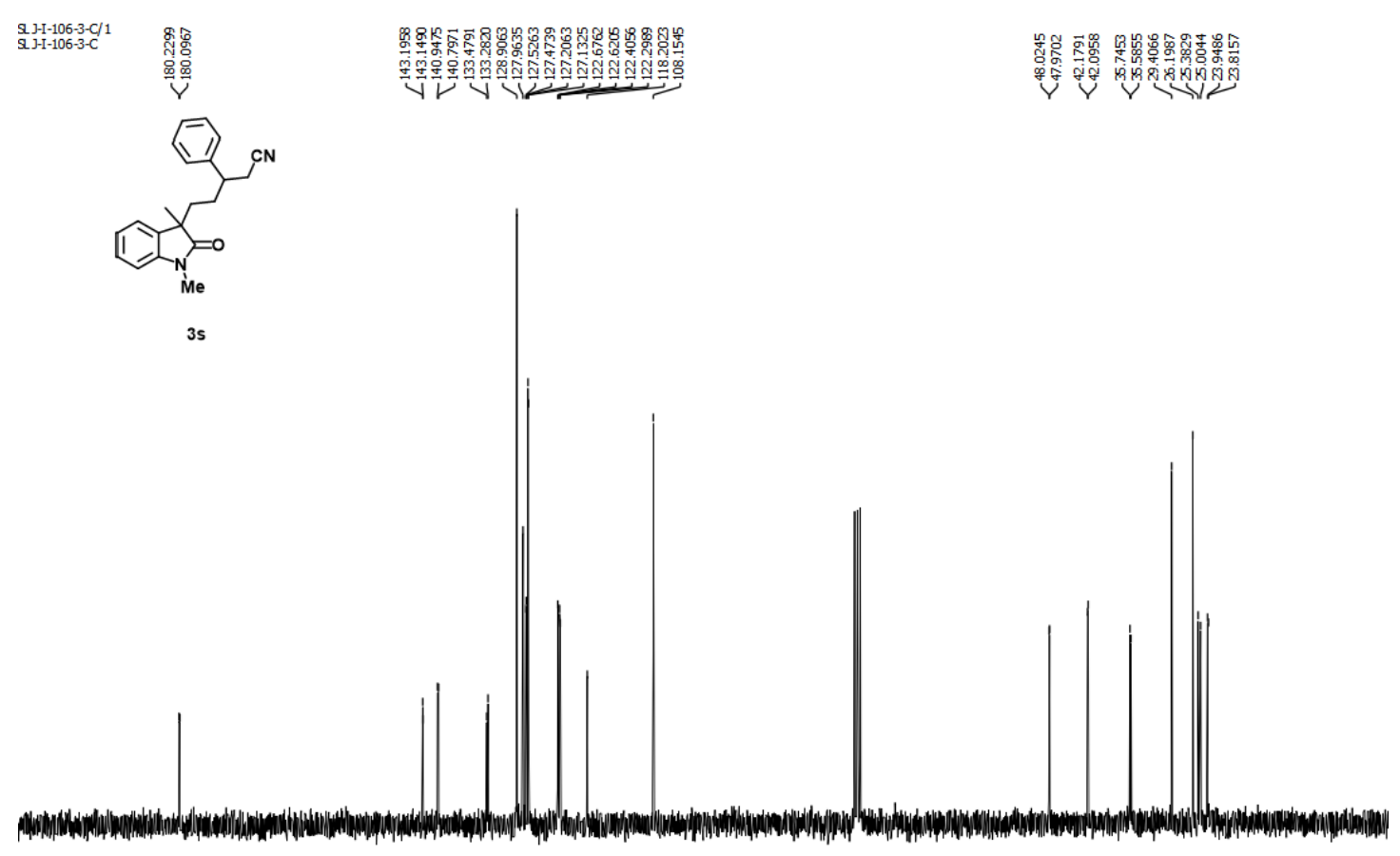

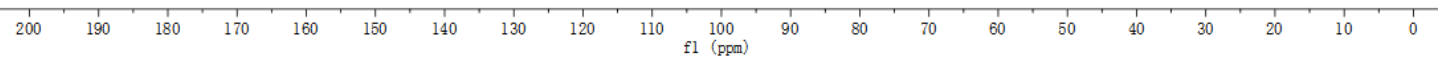




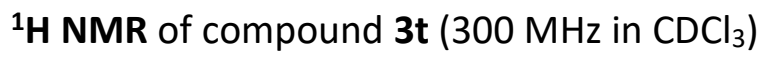

s.]-I-106-4/1

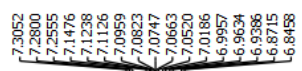

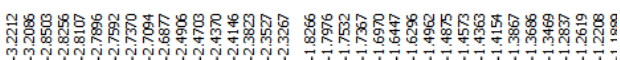

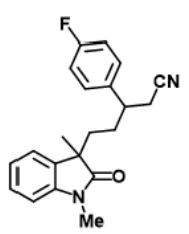

3t

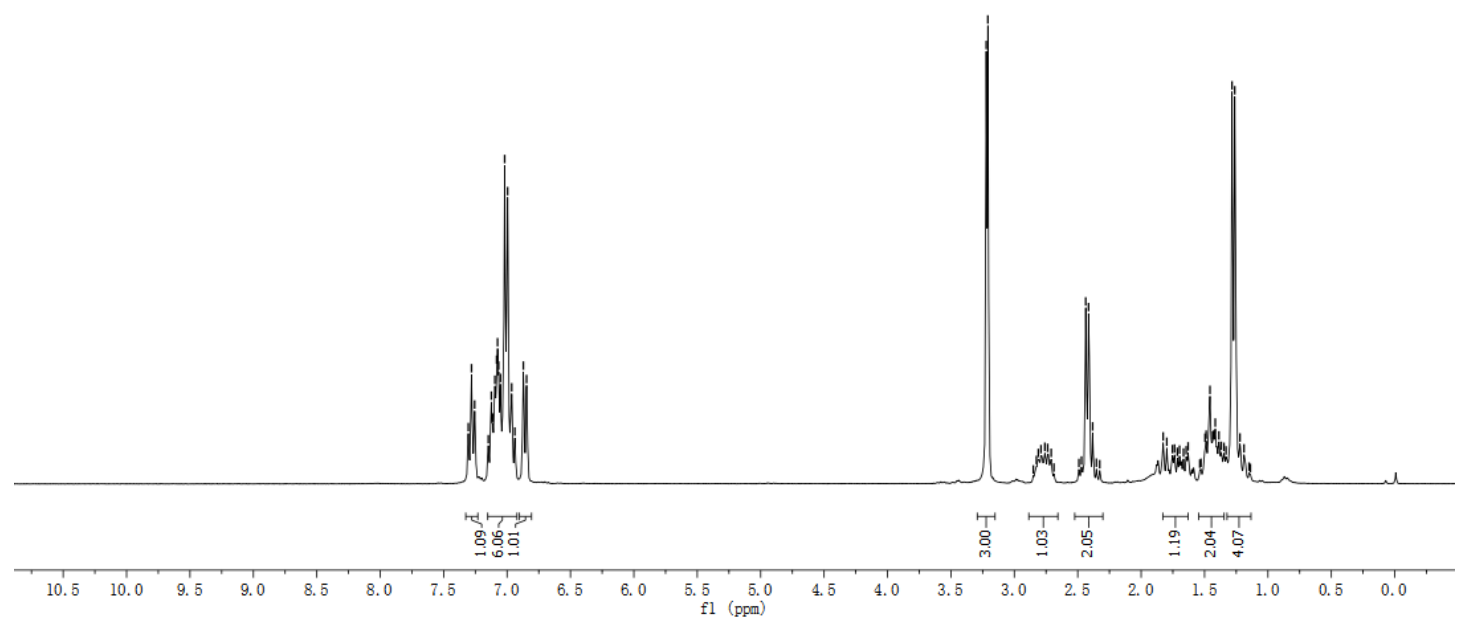

${ }^{13} \mathrm{C}\left\{{ }^{1} \mathrm{H}\right\}$ NMR of compound $3 t\left(75 \mathrm{MHz}\right.$ in $\left.\mathrm{CDCl}_{3}\right)$
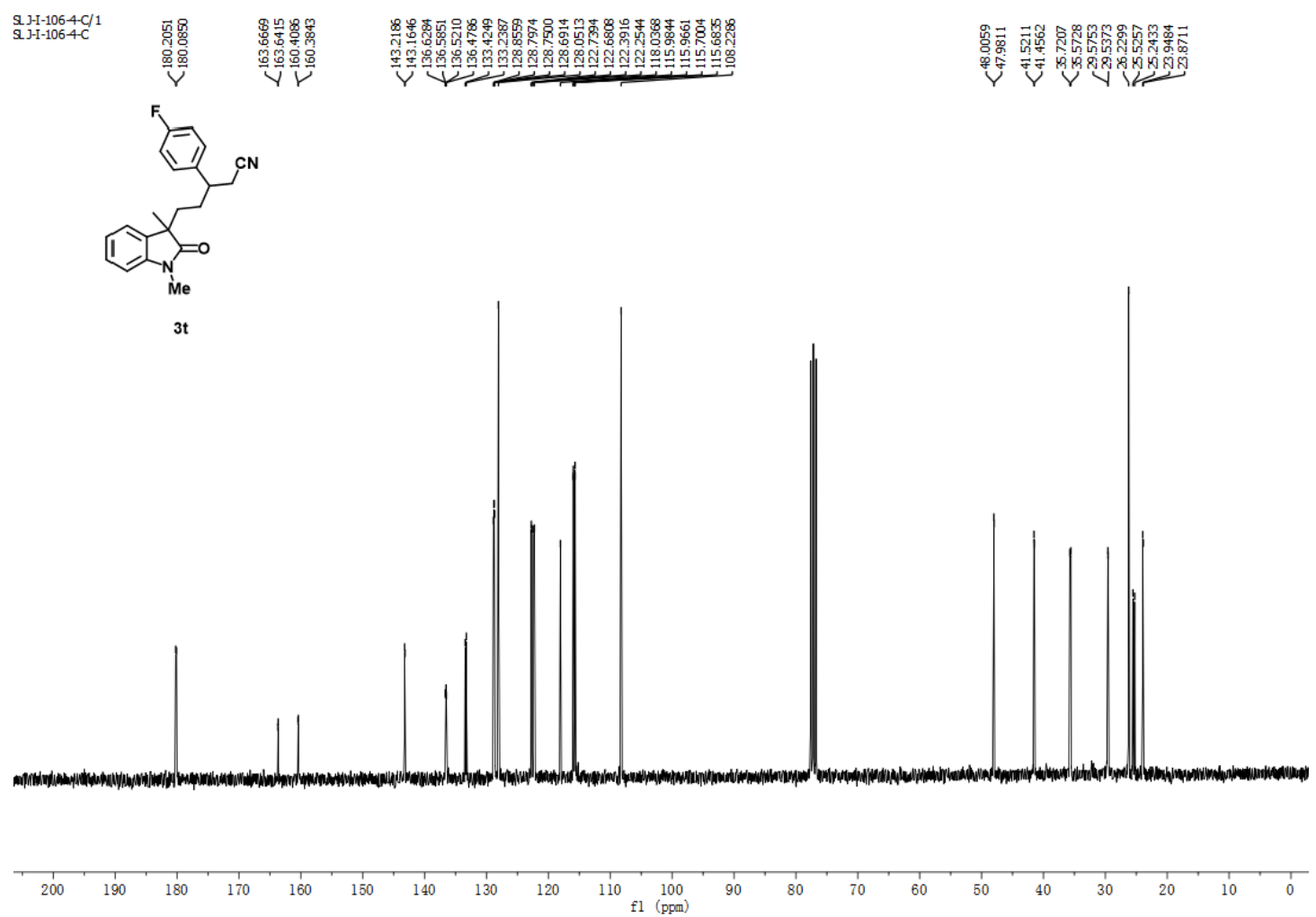
${ }^{19} \mathrm{~F}$ NMR of compound $3 \mathrm{t}\left(282 \mathrm{MHz}\right.$ in $\left.\mathrm{CDCl}_{3}\right)$

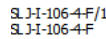

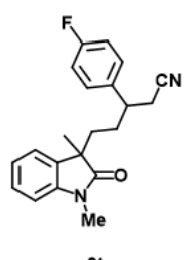

籍

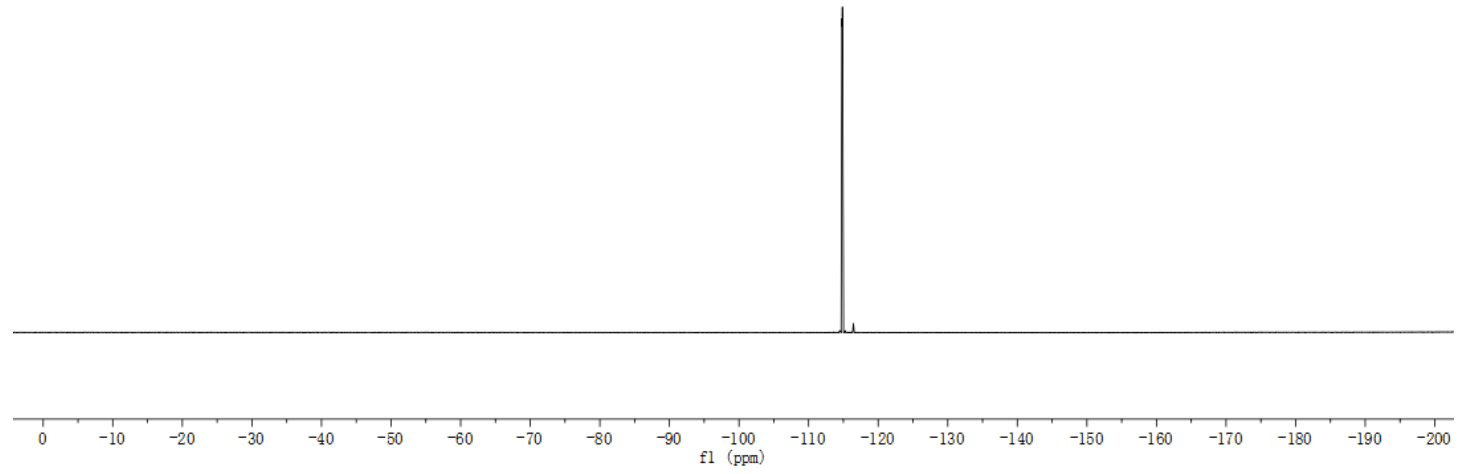

${ }^{{ }^{1} \mathbf{H}}$ NMR of compound $\mathbf{3 u}\left(300 \mathrm{MHz}\right.$ in $\mathrm{CDCl}_{3}$ )

djj-106-5/1

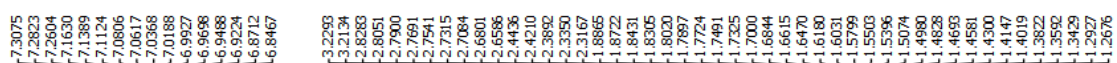

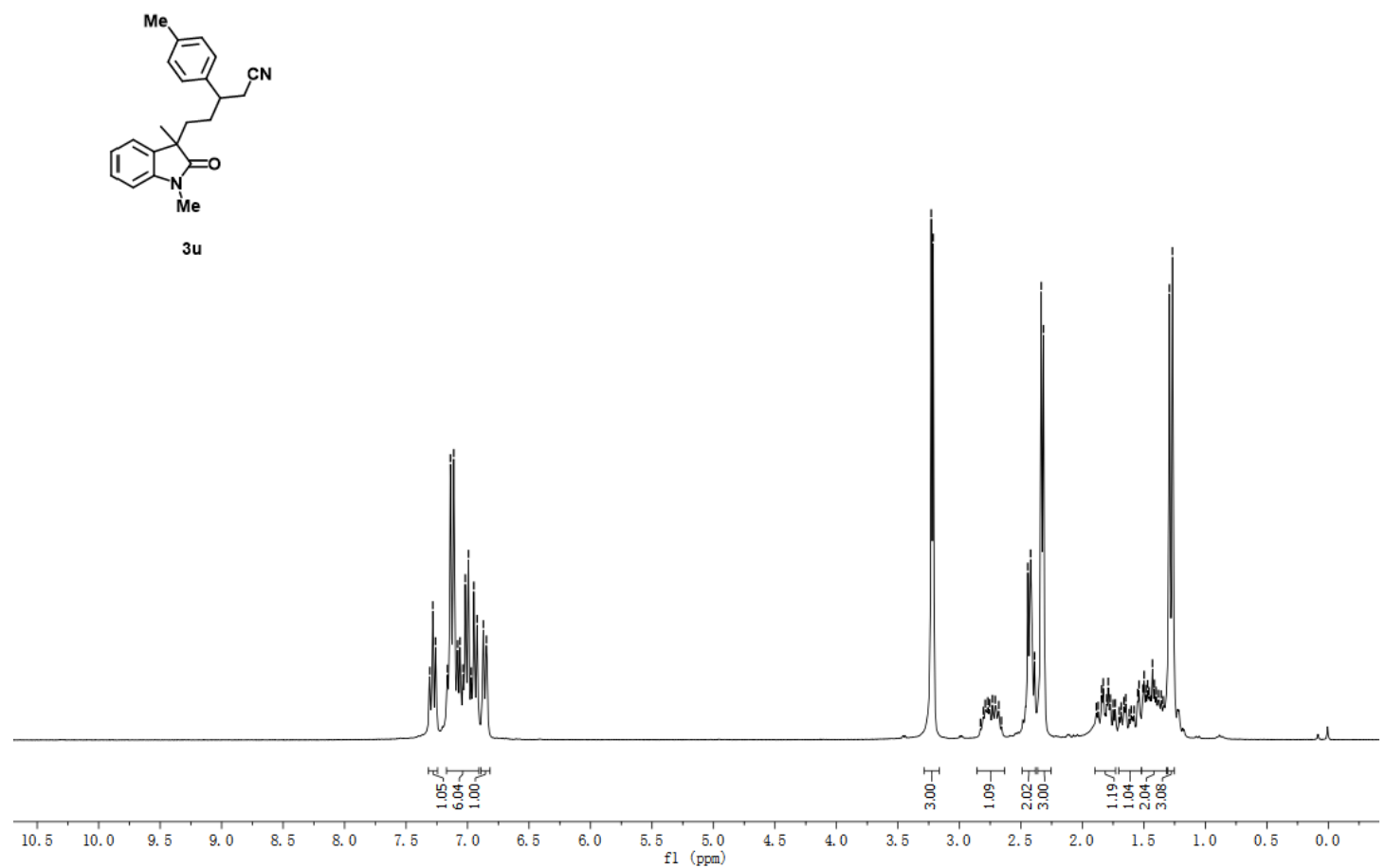


${ }^{13} \mathbf{C}\left\{{ }^{1} \mathrm{H}\right\}$ NMR of compound $\mathbf{3 u}\left(75 \mathrm{MHz}\right.$ in $\mathrm{CDCl}_{3}$ )
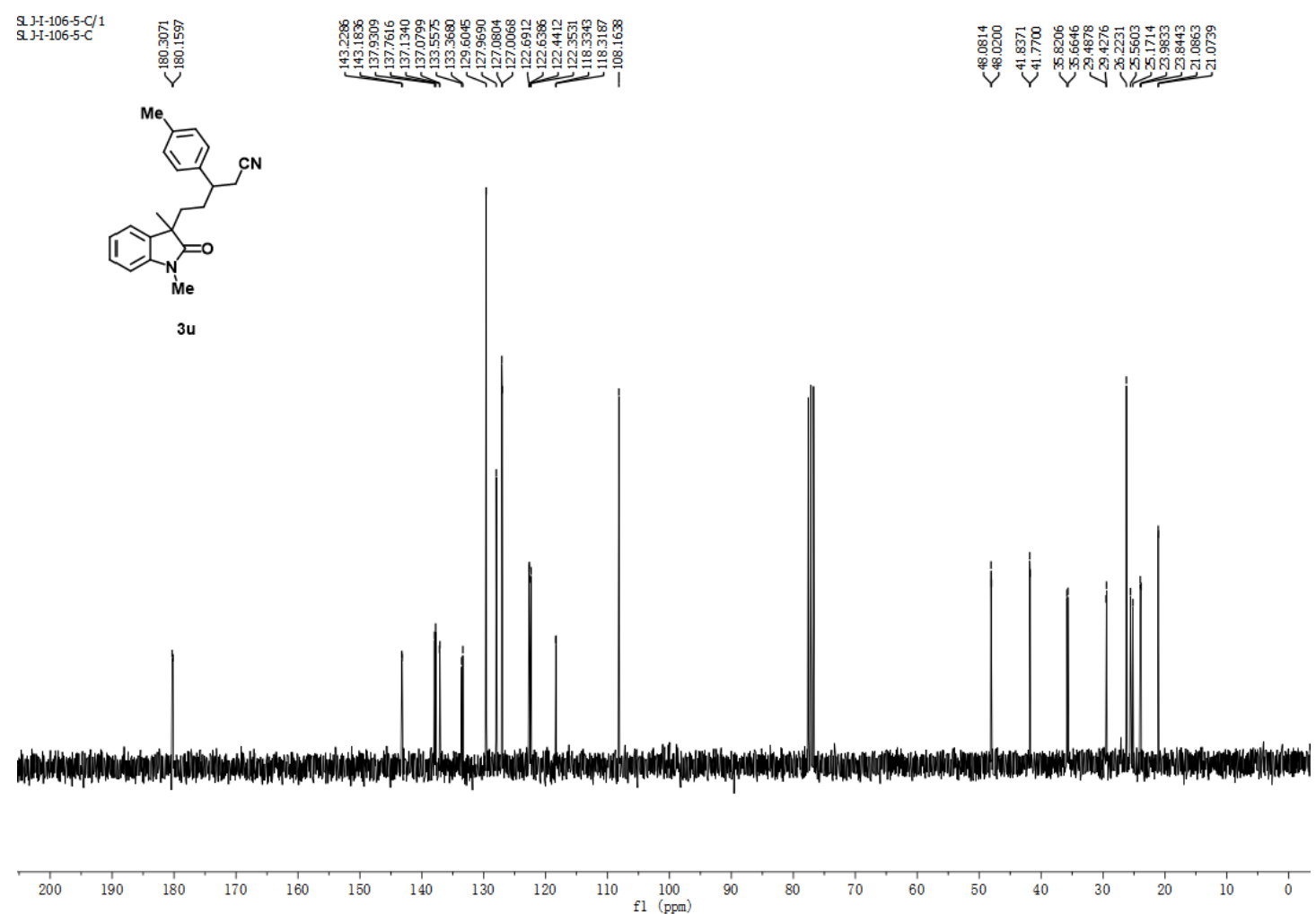

${ }^{1} \mathbf{H}$ NMR of compound $\mathbf{3} \mathbf{v}\left(300 \mathrm{MHz}\right.$ in $\left.\mathrm{CDCl}_{3}\right)$

8. $-1-189-10 / 1$

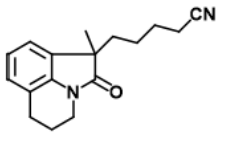

$3 v$

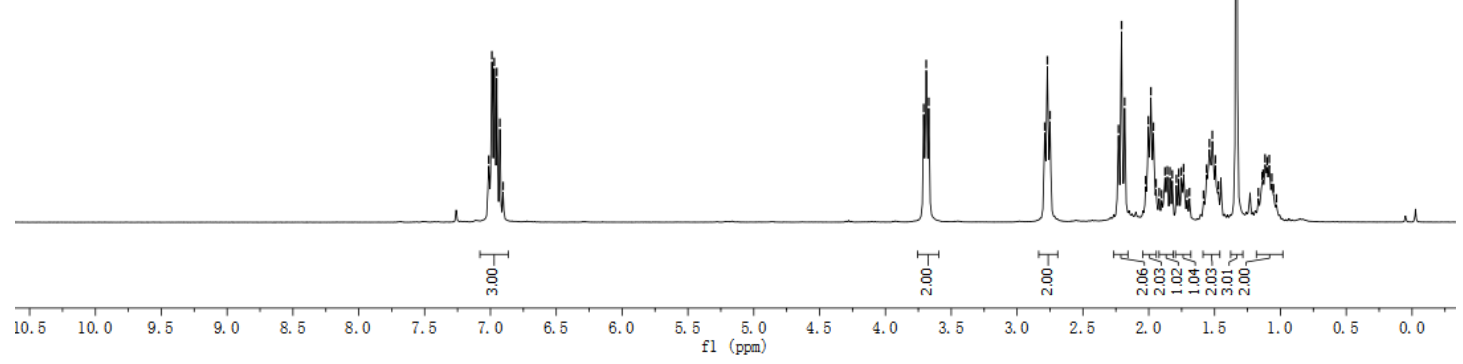


${ }^{13} \mathbf{C}\left\{{ }^{1} \mathrm{H}\right\}$ NMR of compound $3 \mathbf{v}\left(75 \mathrm{MHz}\right.$ in $\left.\mathrm{CDCl}_{3}\right)$
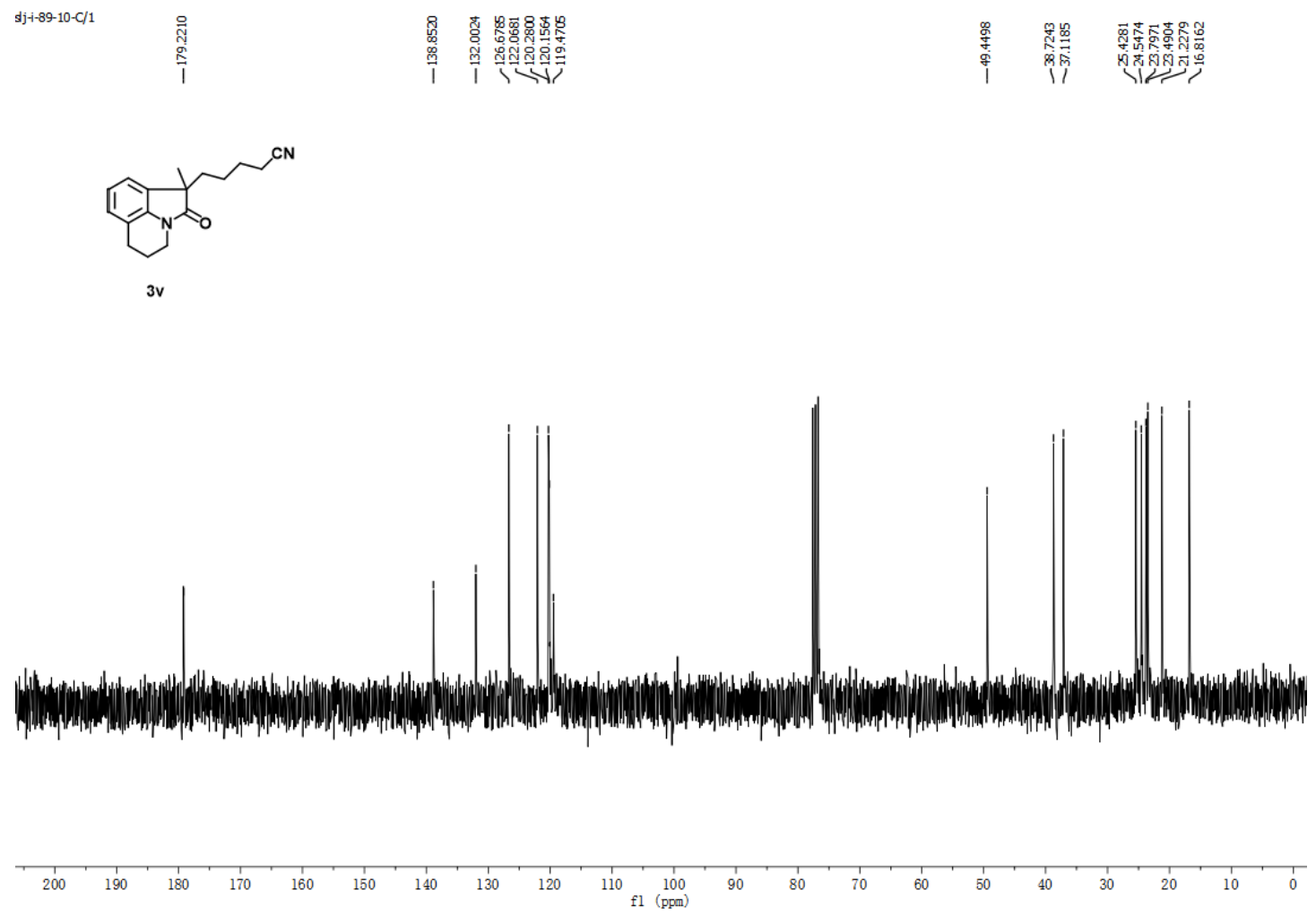

${ }^{1} \mathbf{H}$ NMR of compound 4 a (300 MHz in $\mathrm{CDCl}_{3}$ ) จ. $3-193-4 / 1$
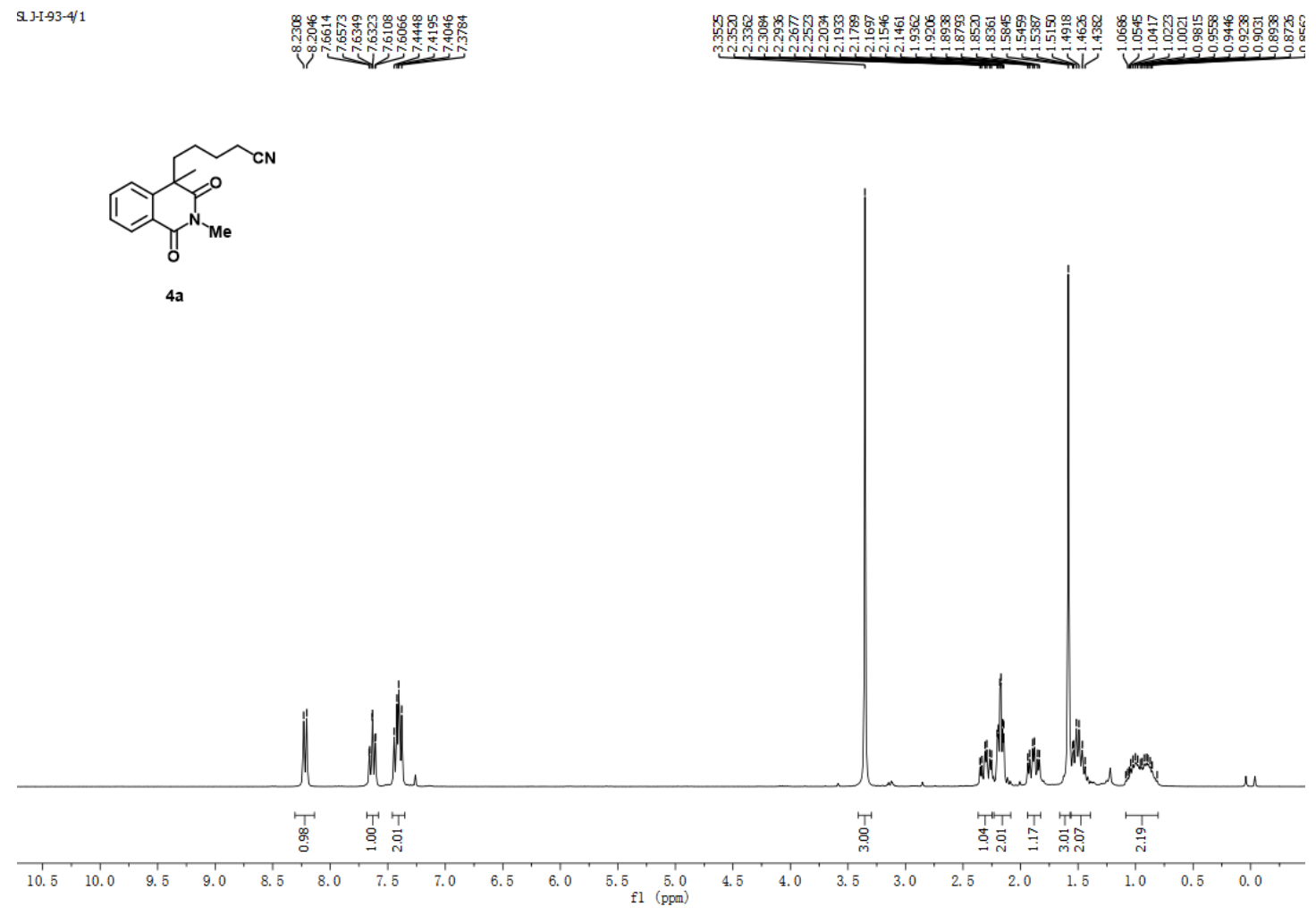
${ }^{13} \mathrm{C}\left\{{ }^{1} \mathrm{H}\right\}$ NMR of compound 4 a $\left(75 \mathrm{MHz}\right.$ in $\mathrm{CDCl}_{3}$ )
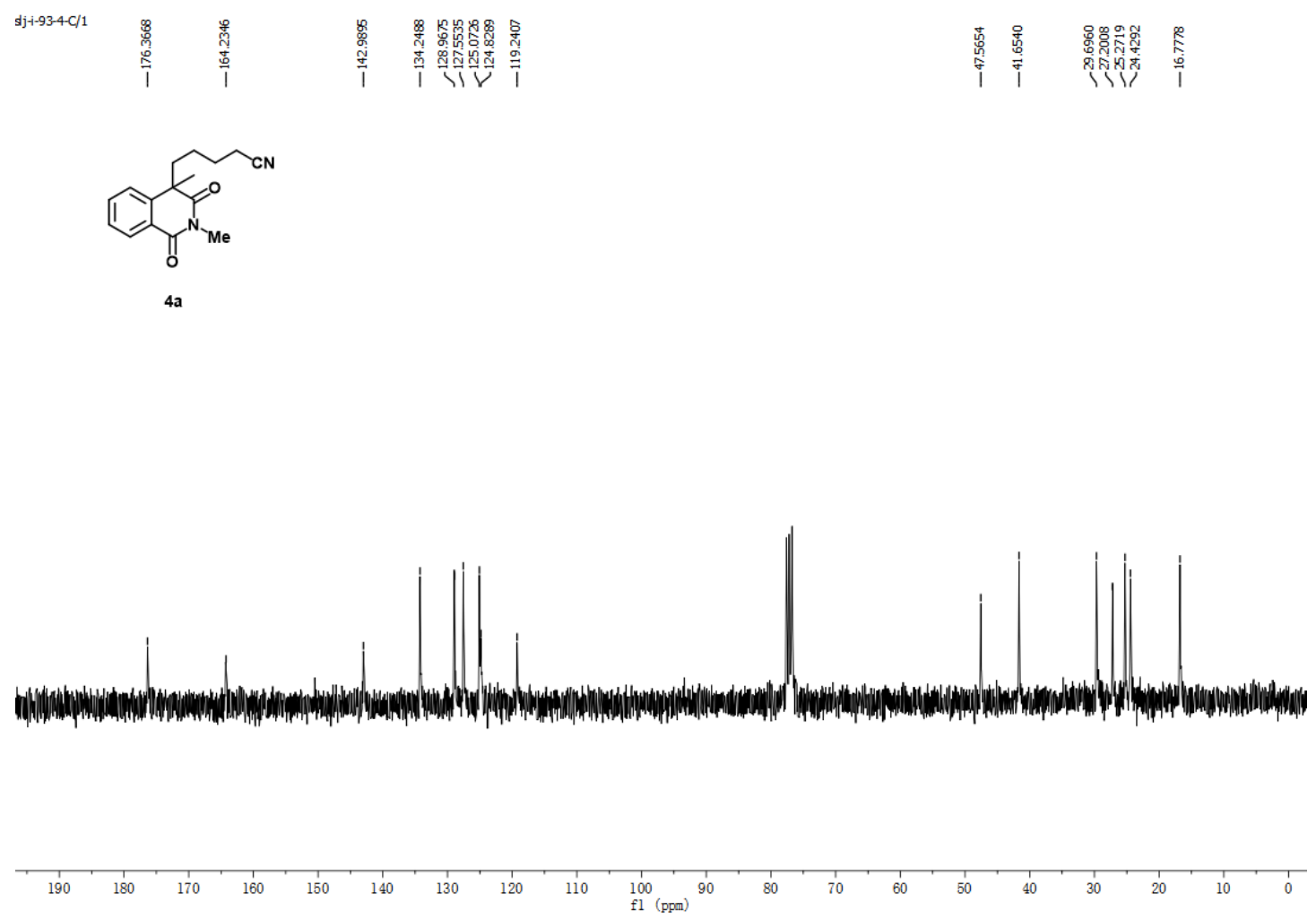

${ }^{1} \mathbf{H}$ NMR of compound $\mathbf{4 b}\left(300 \mathrm{MHz}\right.$ in $\left.\mathrm{CDCl}_{3}\right)$
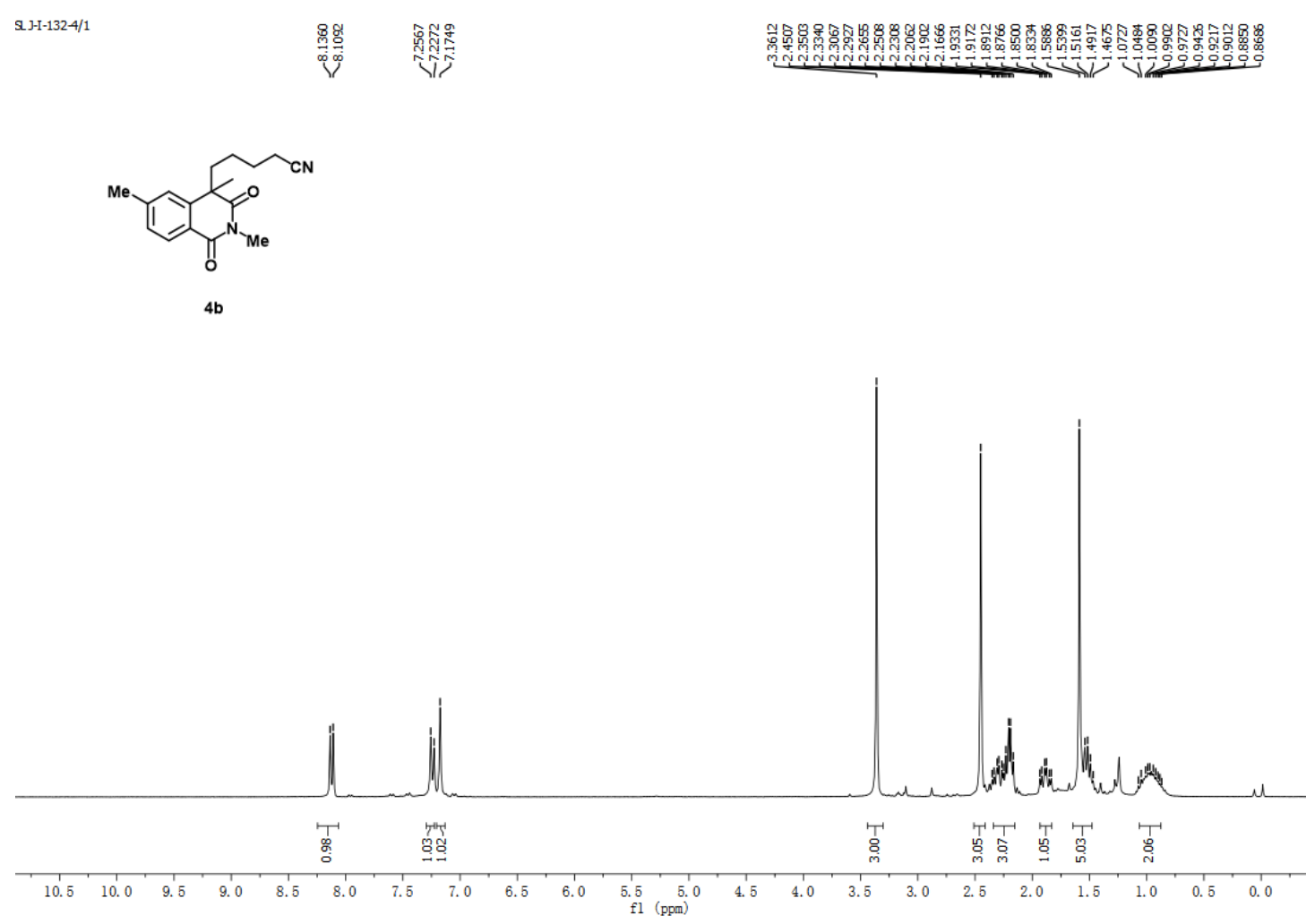
${ }^{13} \mathbf{C}\left\{{ }^{1} \mathrm{H}\right\}$ NMR of compound $\mathbf{4 b}\left(75 \mathrm{MHz}\right.$ in $\mathrm{CDCl}_{3}$ )
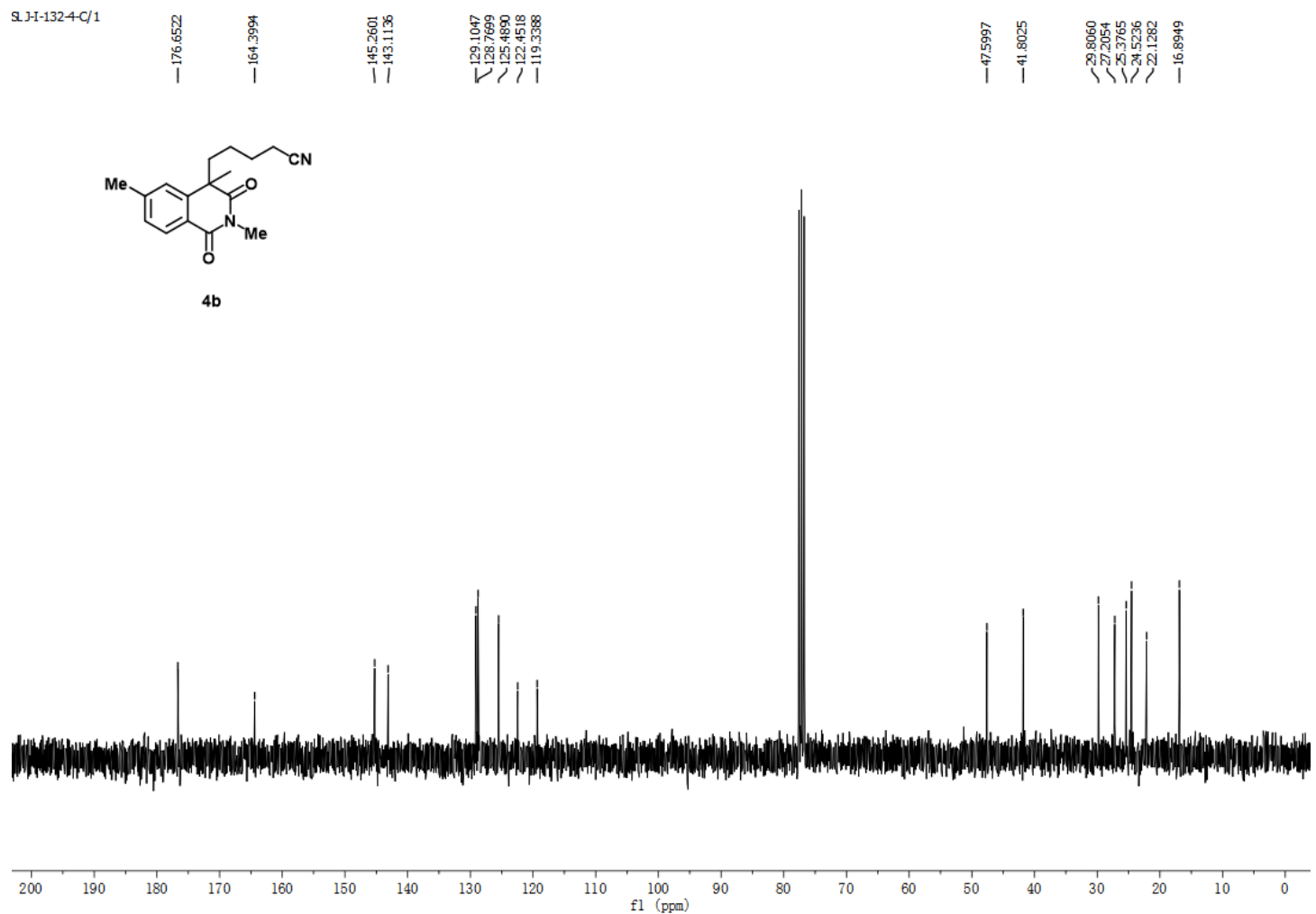

${ }^{1} \mathbf{H}$ NMR of compound $\mathbf{4 c}\left(300 \mathrm{MHz}\right.$ in $\left.\mathrm{CDCl}_{3}\right)$

s.]-I-1326/1

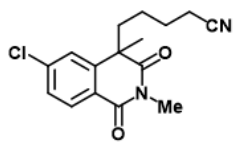

$4 c$

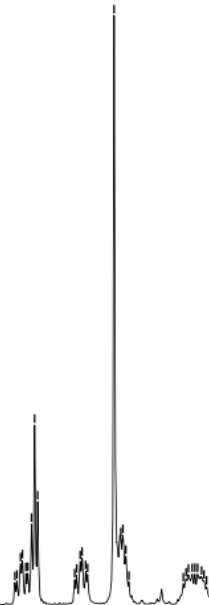

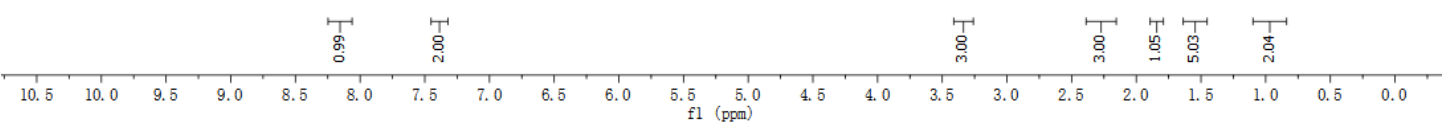


${ }^{13} \mathrm{C}\left\{{ }^{1} \mathrm{H}\right\}$ NMR of compound $4 \mathrm{c}\left(75 \mathrm{MHz}\right.$ in $\left.\mathrm{CDCl}_{3}\right)$
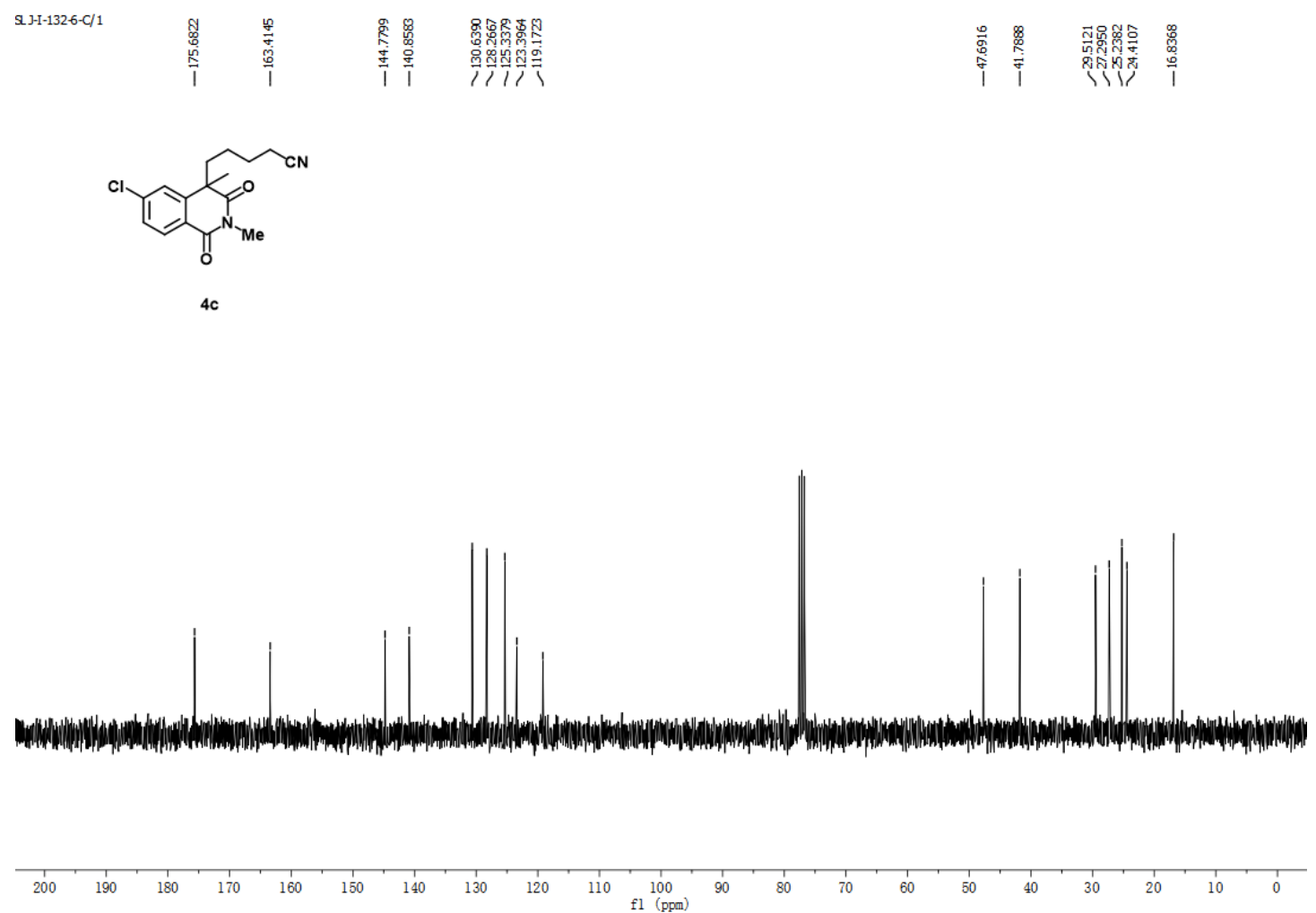

${ }^{{ }^{1}} \mathbf{H}$ NMR of compound $\mathbf{4 d}\left(300 \mathrm{MHz}\right.$ in $\left.\mathrm{CDCl}_{3}\right)$

s.]--1-133-3/1

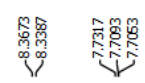

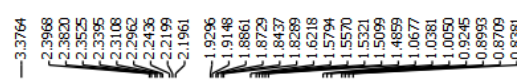

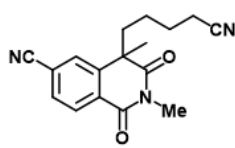

$4 d$

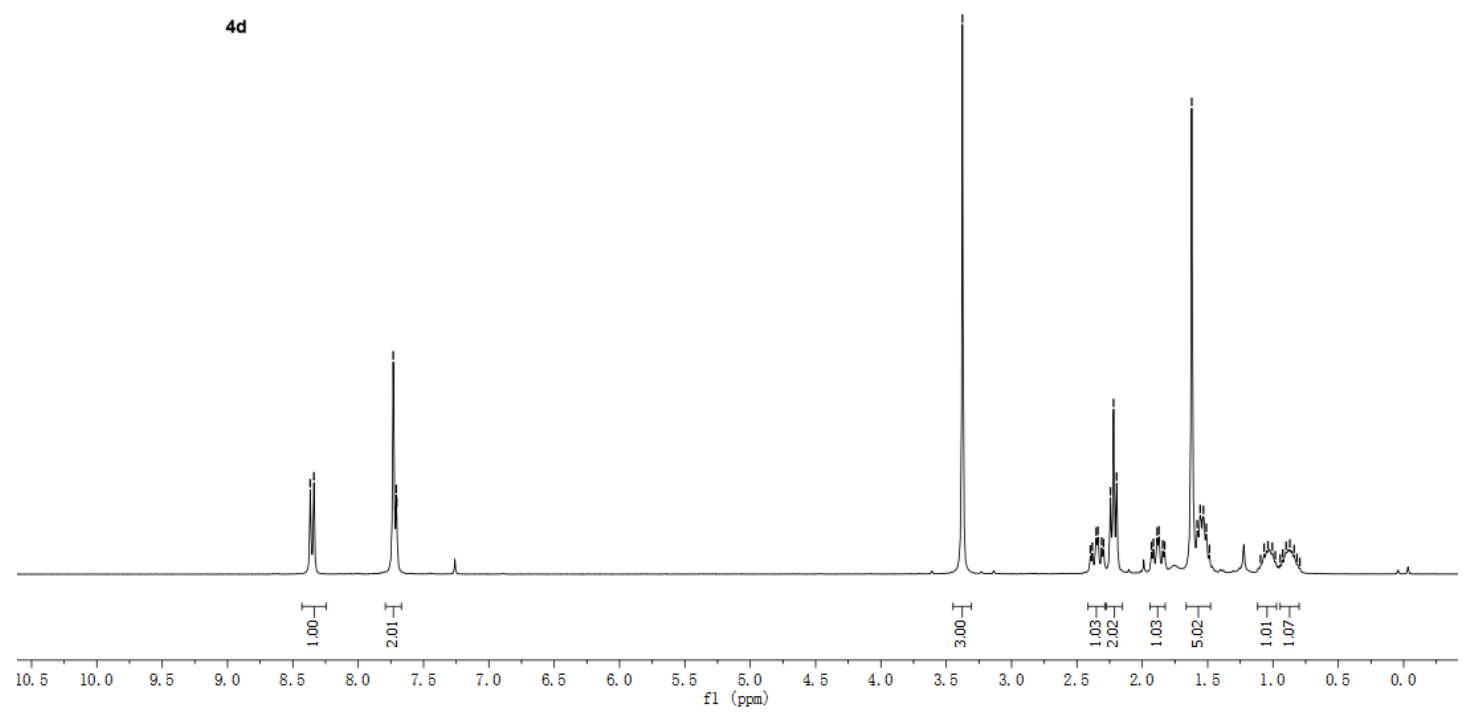


${ }^{13} \mathrm{C}\left\{{ }^{1} \mathrm{H}\right\}$ NMR of compound $4 \mathbf{d}\left(75 \mathrm{MHz}\right.$ in $\left.\mathrm{CDCl}_{3}\right)$
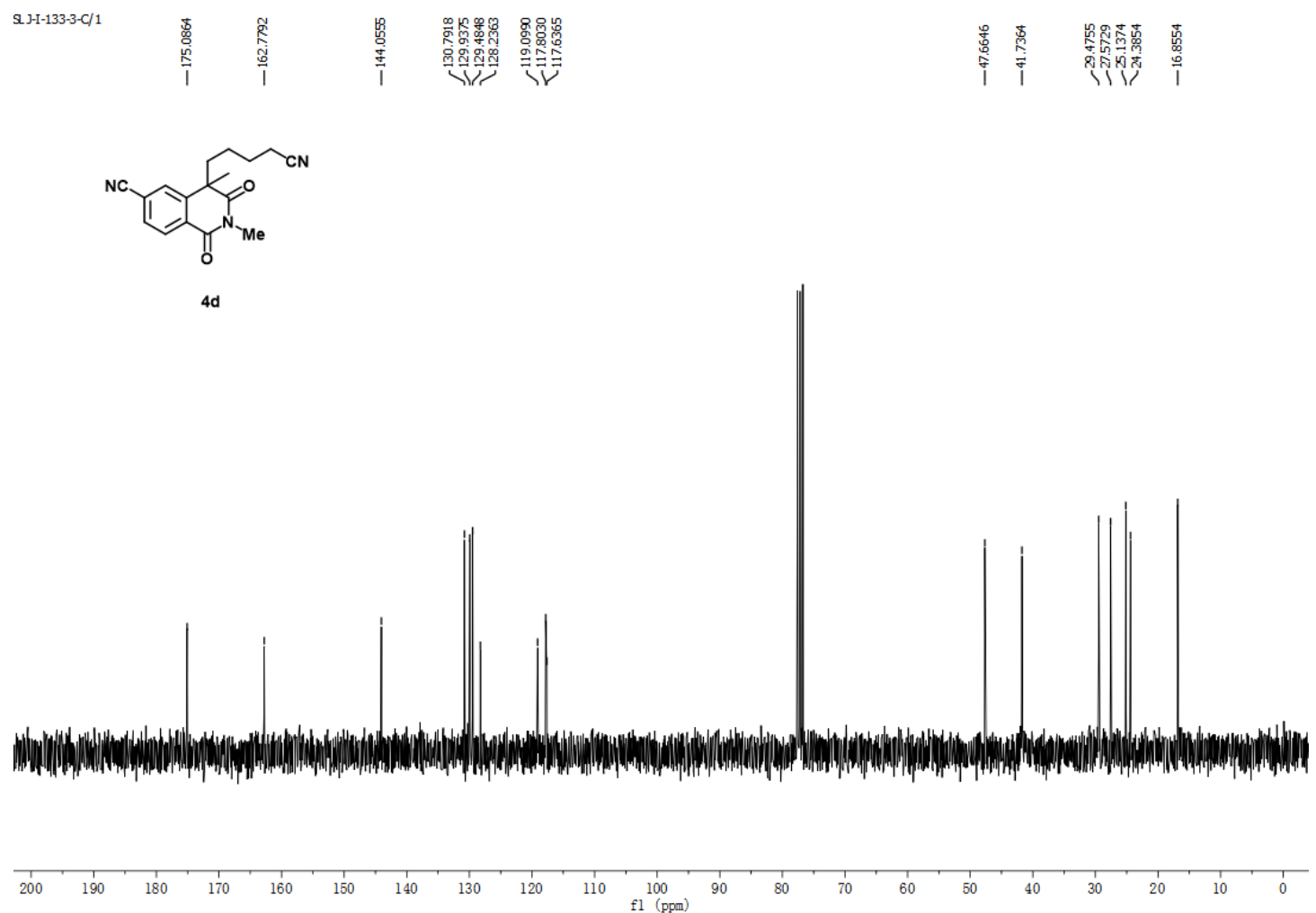

${ }^{1} \mathbf{H}$ NMR of compound $4 \mathbf{e}\left(300 \mathrm{MHz}\right.$ in $\left.\mathrm{CDCl}_{3}\right)$

s.]-I-128-2/1

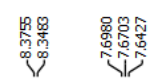

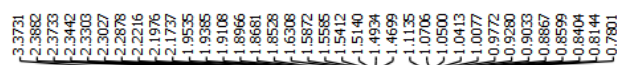

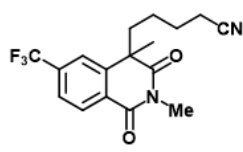

$4 e$

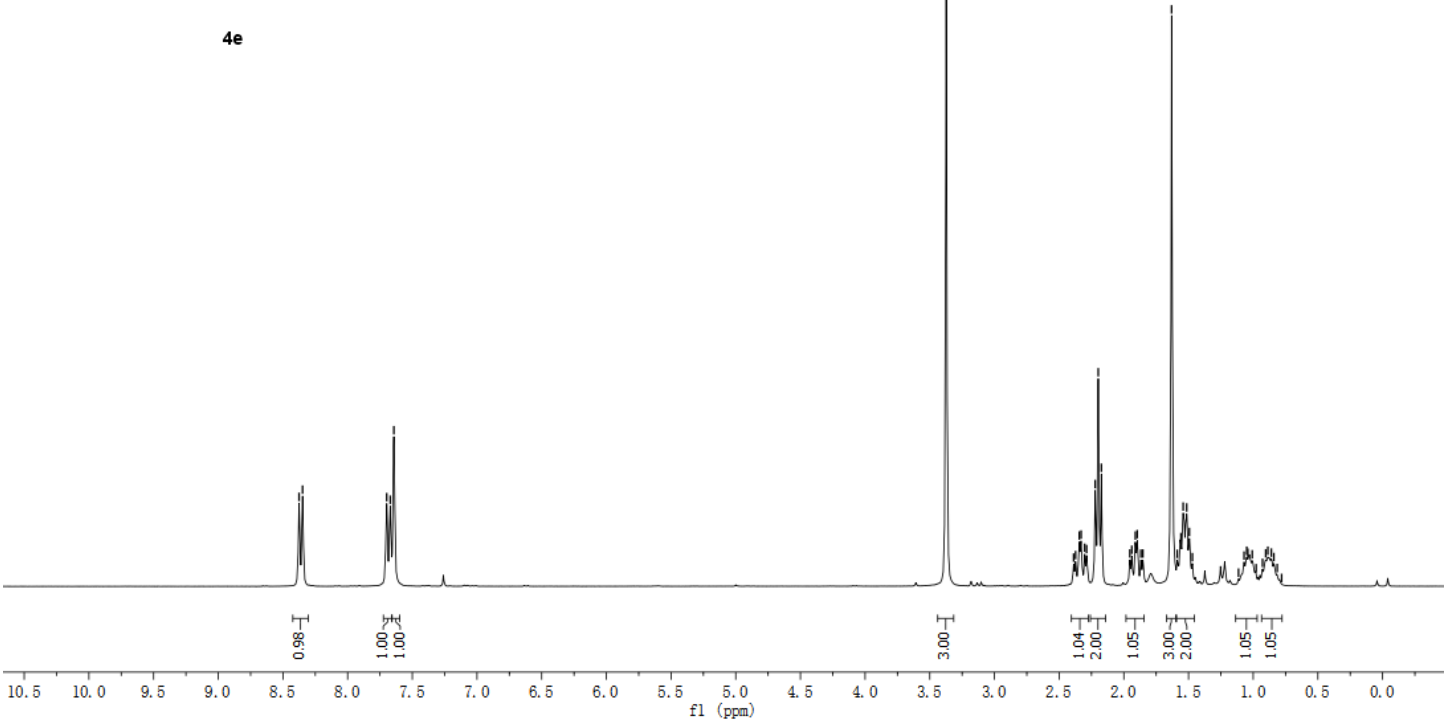


${ }^{13} \mathrm{C}\left\{{ }^{1} \mathrm{H}\right\}$ NMR of compound $4 \mathrm{e}\left(75 \mathrm{MHz}\right.$ in $\left.\mathrm{CDCl}_{3}\right)$
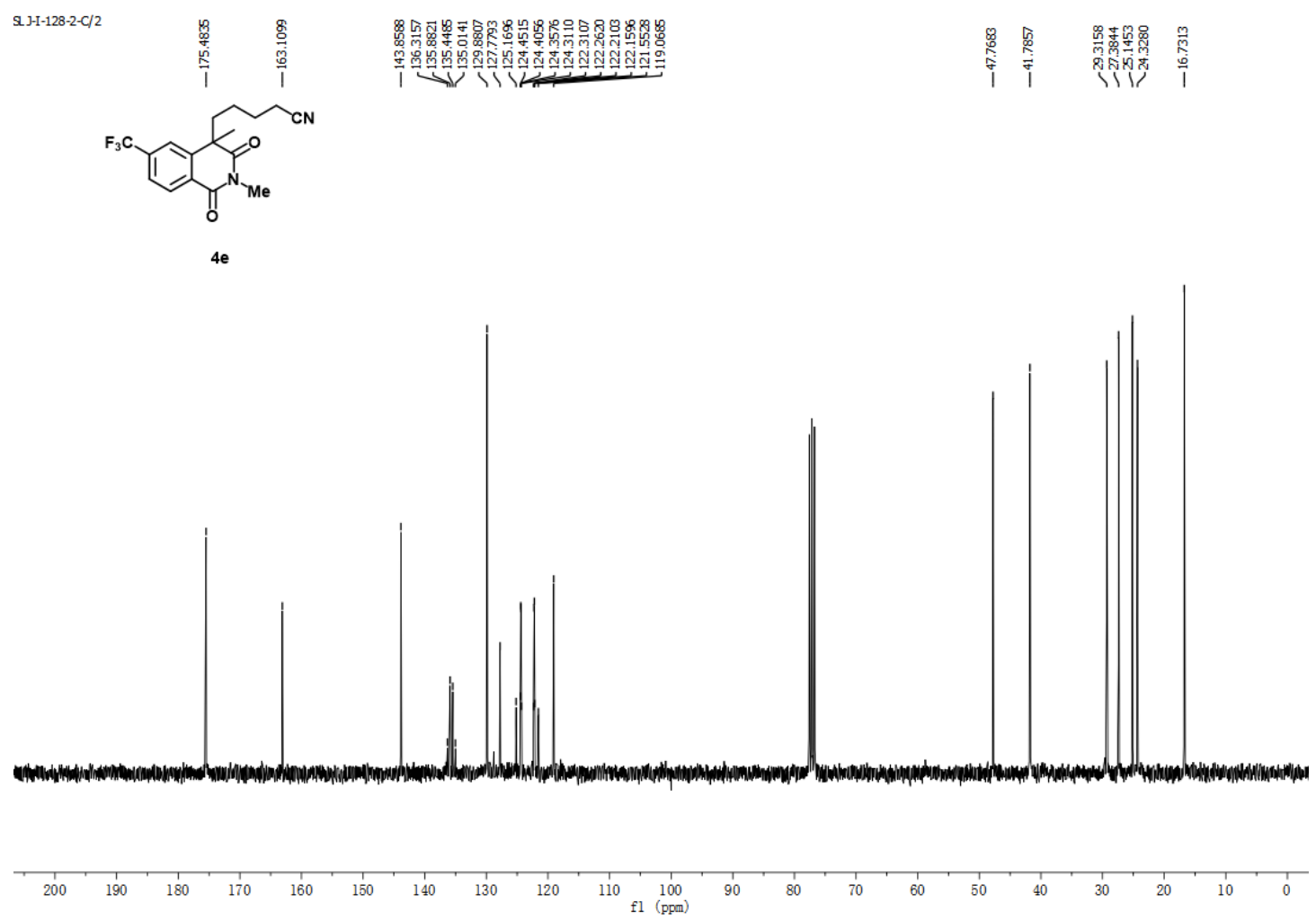

${ }^{19} \mathrm{~F}$ NMR of compound $4 \mathrm{e}\left(282 \mathrm{MHz}\right.$ in $\left.\mathrm{CDCl}_{3}\right)$

8. $-\mathrm{I}-128-2-\mathrm{F} / 1$

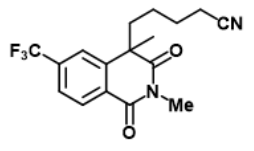

$4 e$

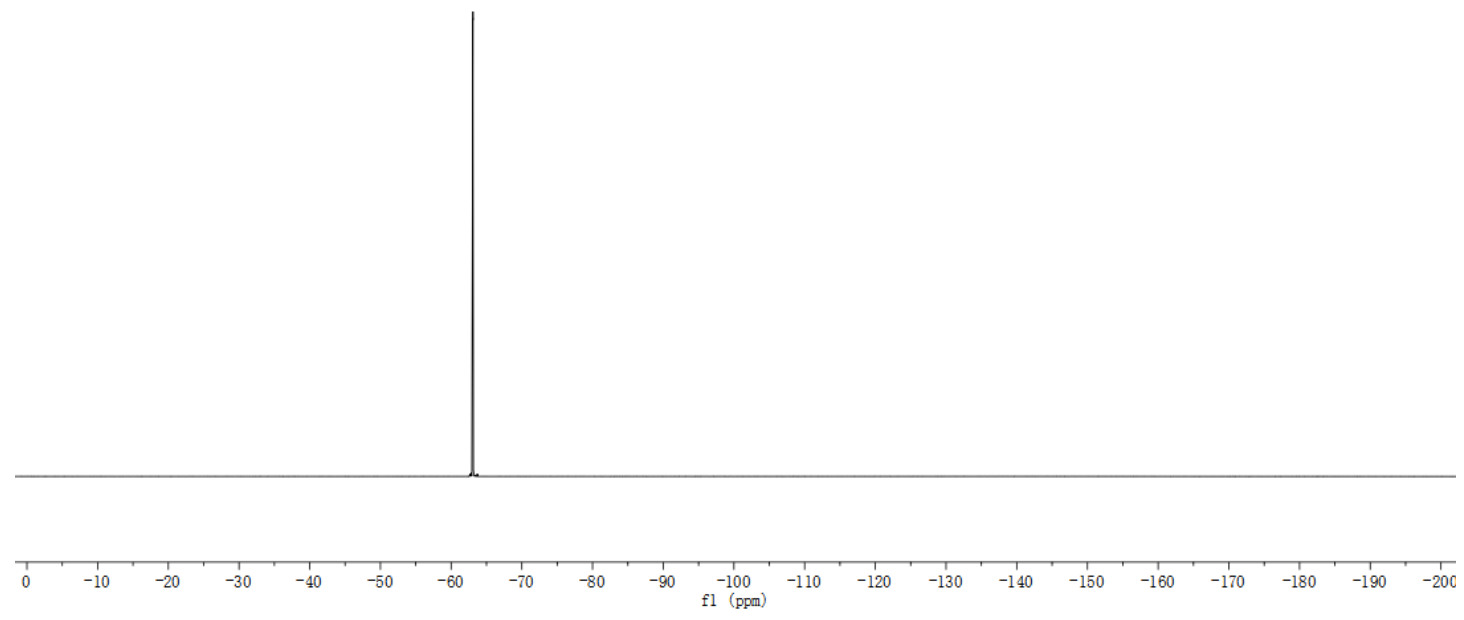


${ }^{\mathbf{1}} \mathbf{H}$ NMR of compound $\mathbf{4 f}\left(300 \mathrm{MHz}\right.$ in $\left.\mathrm{CDCl}_{3}\right)$

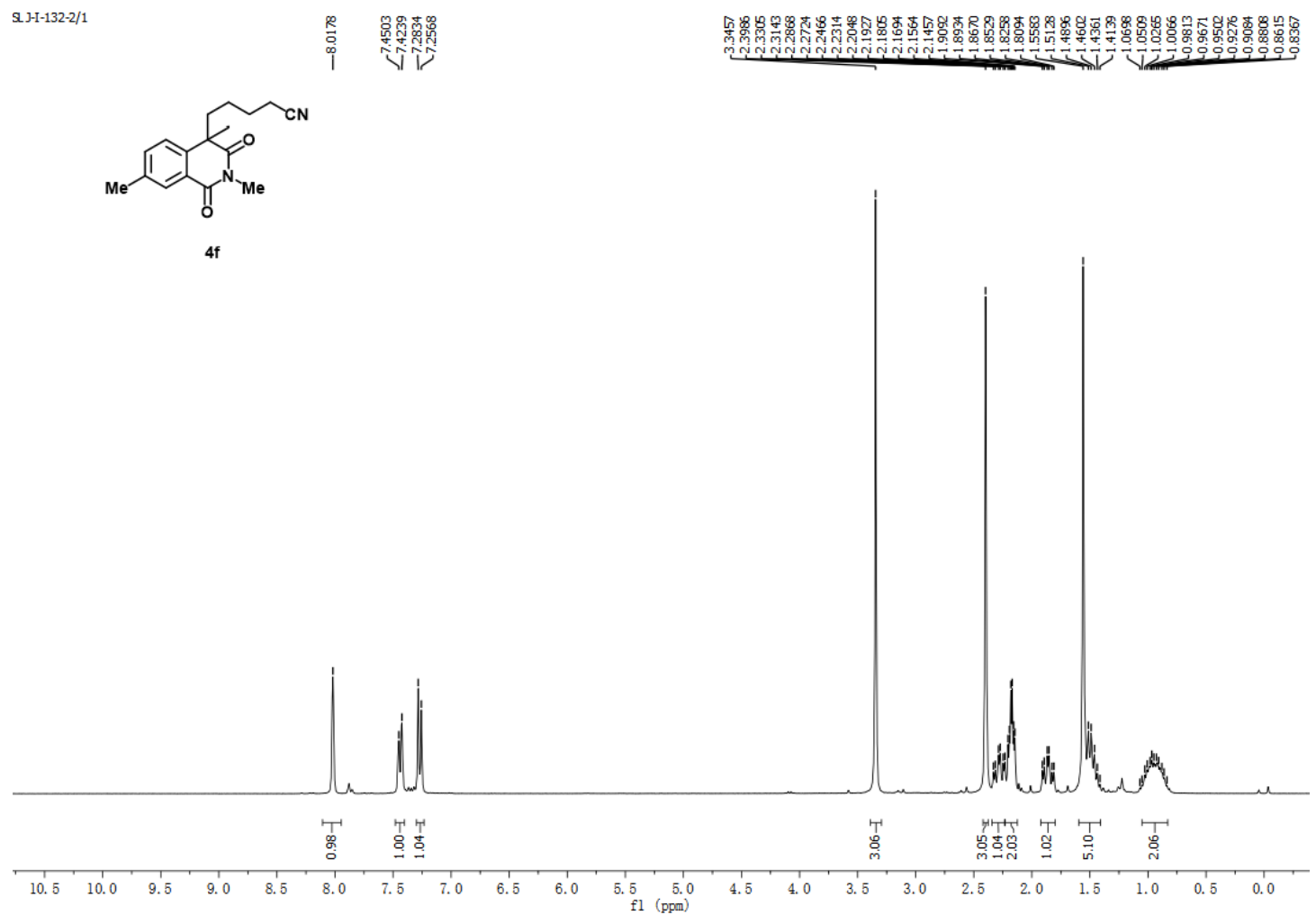

${ }^{13} \mathrm{C}\left\{{ }^{1} \mathrm{H}\right\} \mathrm{NMR}$ of compound $\mathbf{4 f}\left(75 \mathrm{MHz}\right.$ in $\left.\mathrm{CDCl}_{3}\right)$

s. $]-\mathrm{I}-132-2-\mathrm{C} / 1$

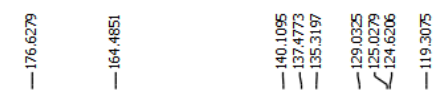

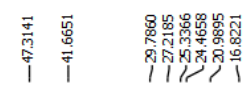

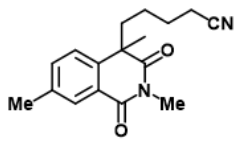

4 f

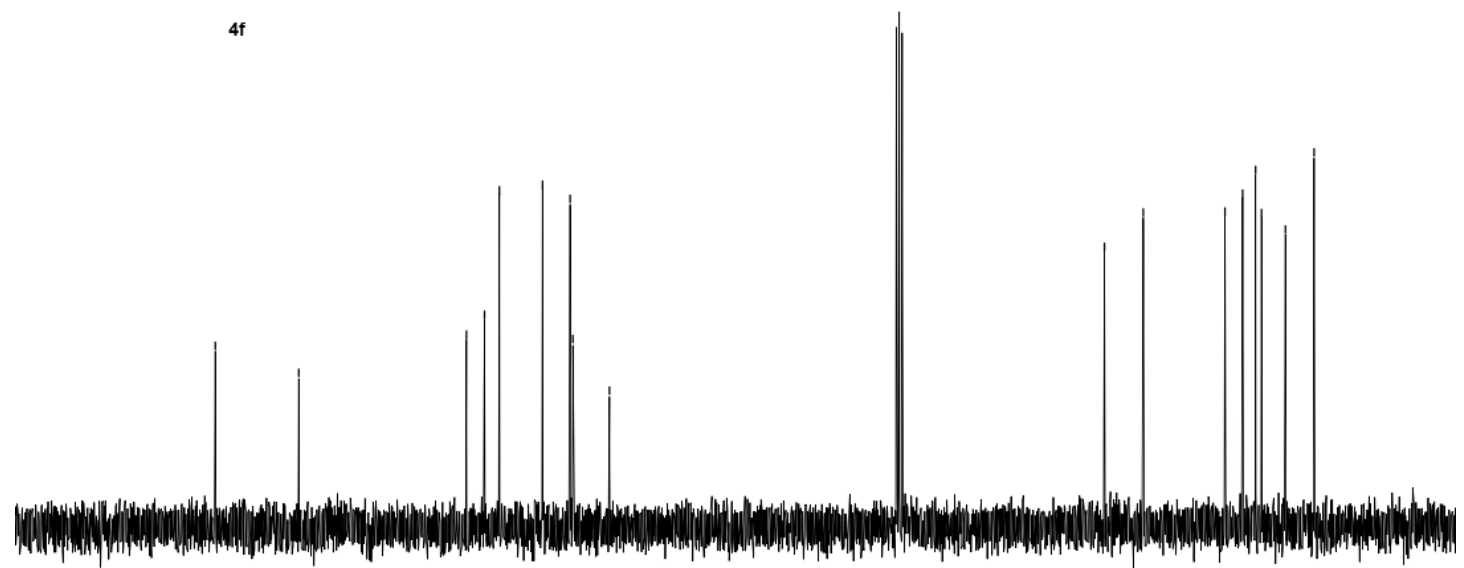

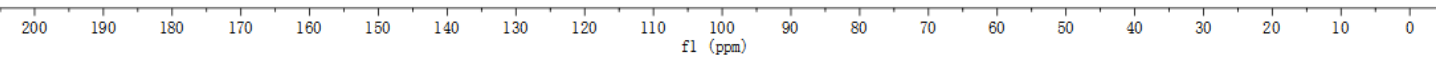


${ }^{1} \mathbf{H}$ NMR of compound $\mathbf{4 g}\left(300 \mathrm{MHz}\right.$ in $\left.\mathrm{CDCl}_{3}\right)$

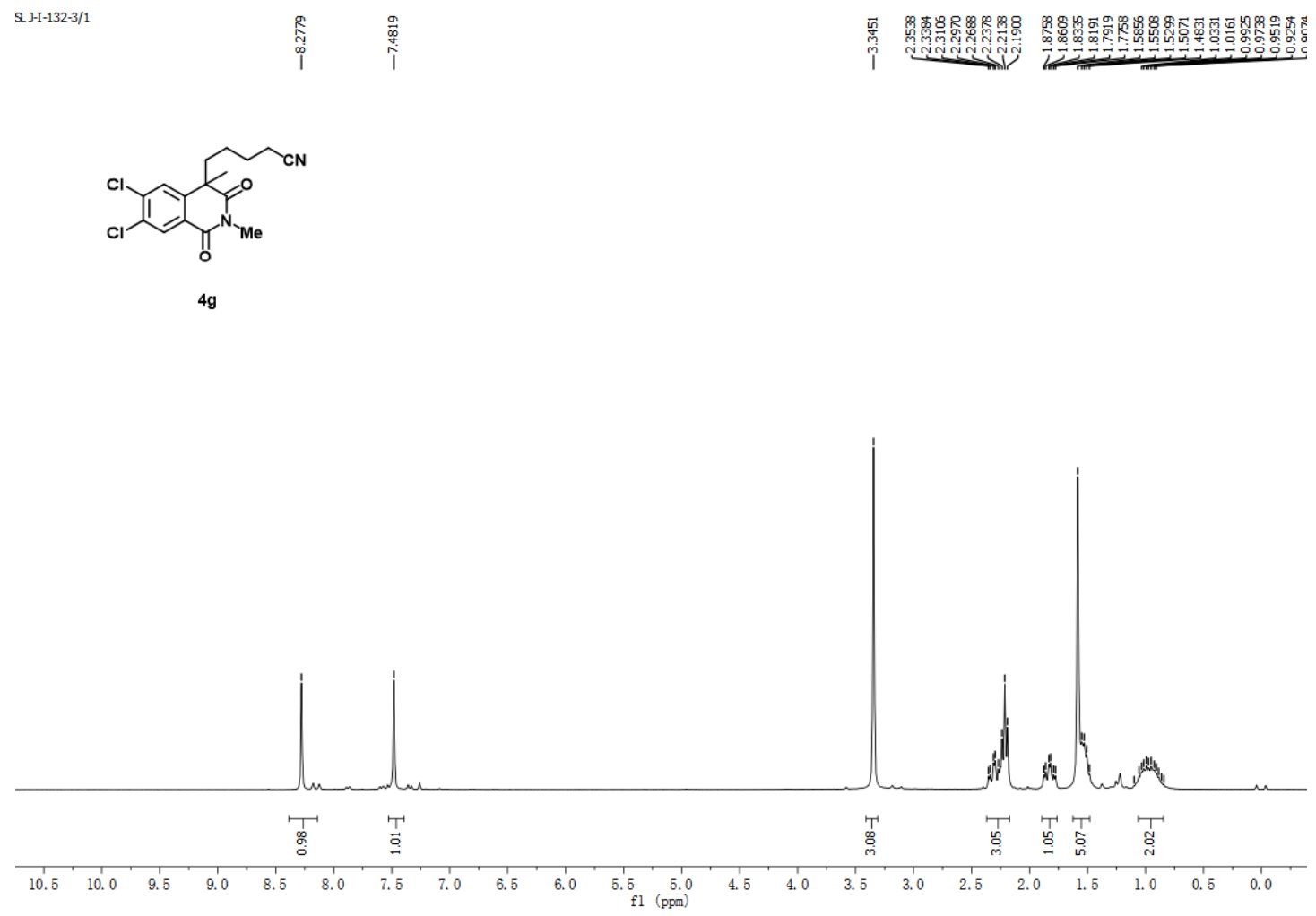

${ }^{13} \mathrm{C}\left\{{ }^{1} \mathrm{H}\right\}$ NMR of compound $\mathbf{4 g}\left(75 \mathrm{MHz}\right.$ in $\left.\mathrm{CDCl}_{3}\right)$

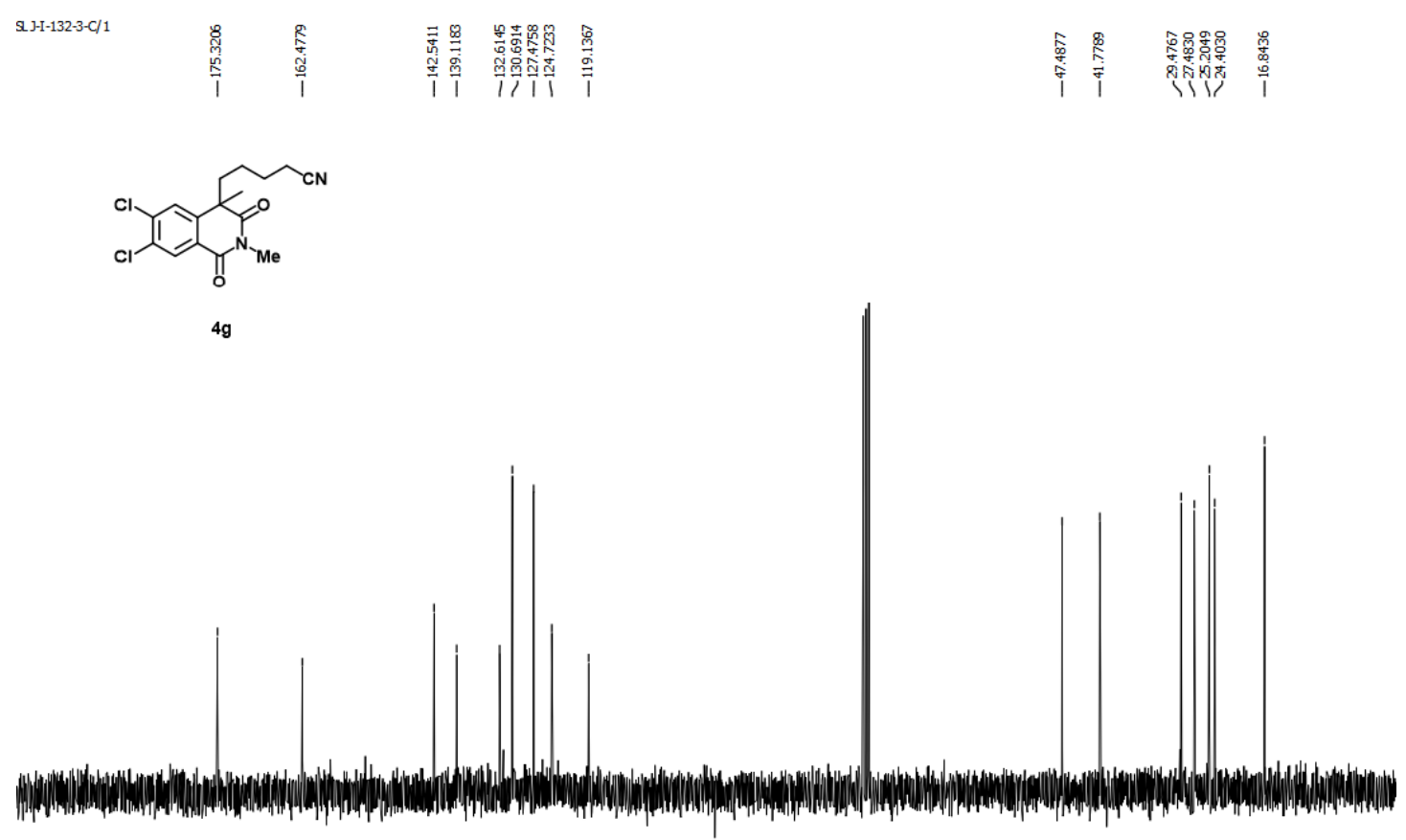

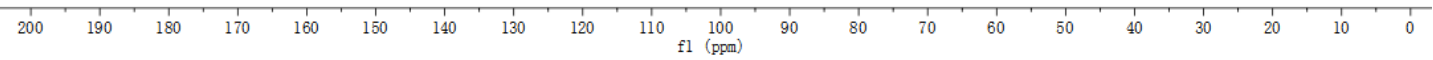


${ }^{1} \mathbf{H}$ NMR of compound 5 a $\left(300 \mathrm{MHz}\right.$ in $\left.\mathrm{CDCl}_{3}\right)$

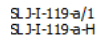

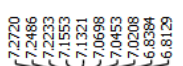

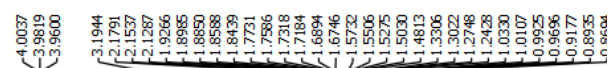

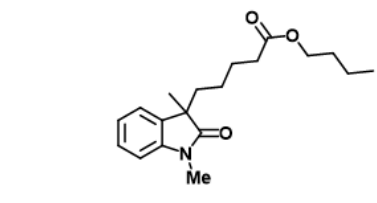

$5 a$

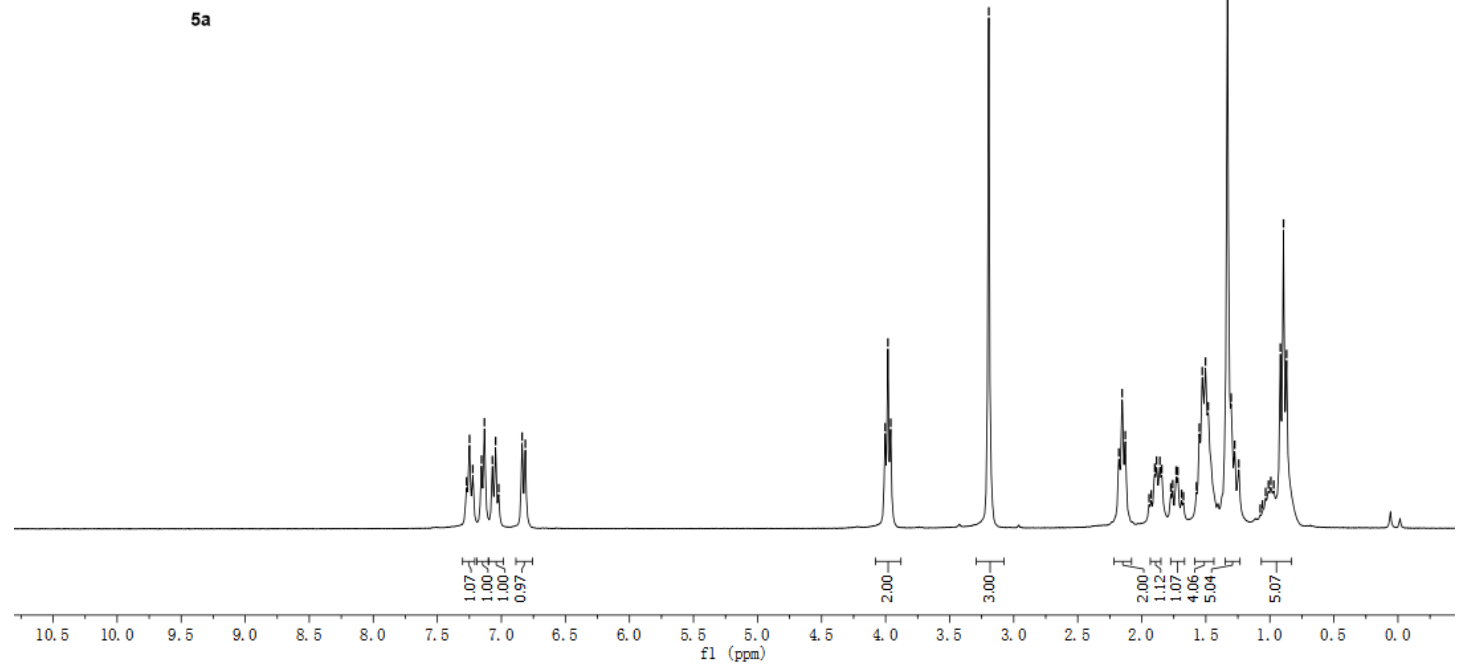

${ }^{13} \mathrm{C}\left\{{ }^{1} \mathrm{H}\right\}$ NMR of compound $\mathbf{5 a}\left(75 \mathrm{MHz}\right.$ in $\left.\mathrm{CDCl}_{3}\right)$

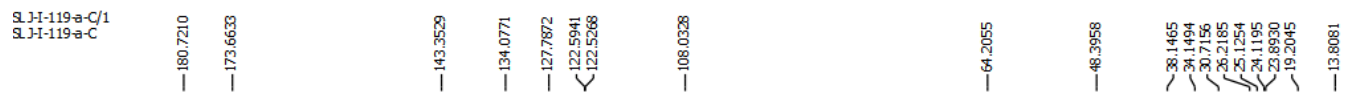

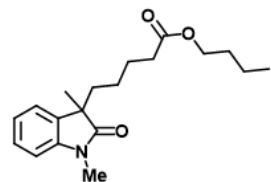

$5 a$

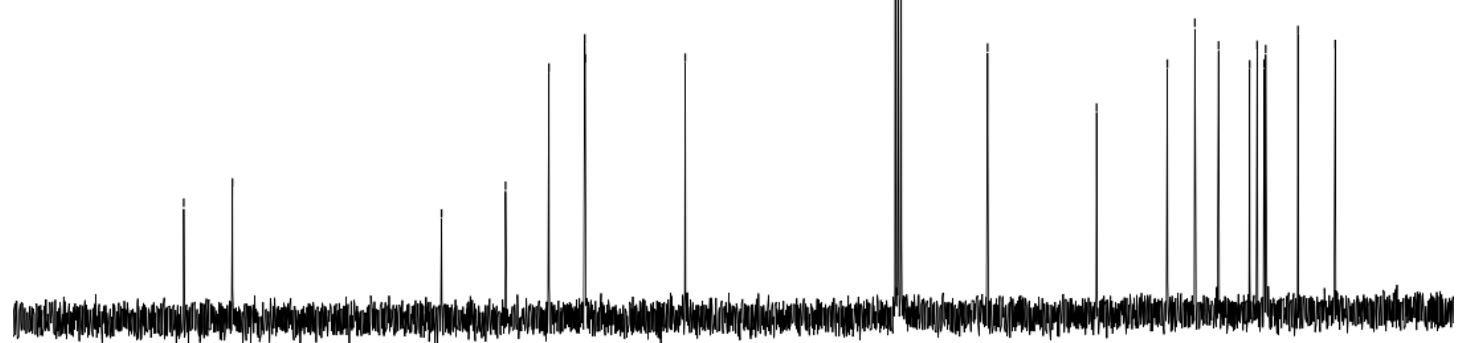

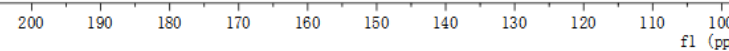


${ }^{1} \mathbf{H}$ NMR of compound $\mathbf{5 b}\left(300 \mathrm{MHz}\right.$ in $\left.\mathrm{CDCl}_{3}\right)$

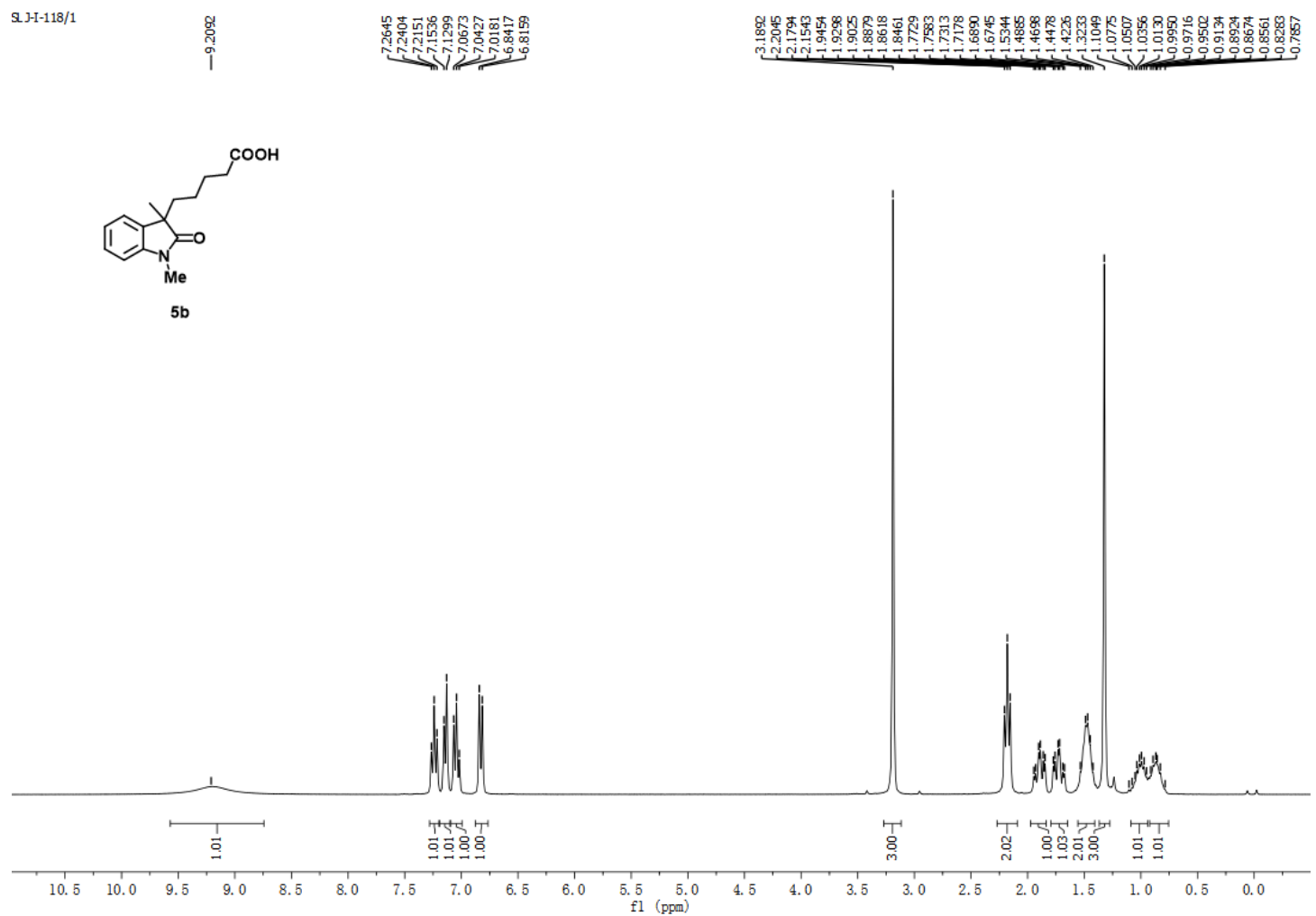

${ }^{13} \mathbf{C}\left\{{ }^{1} \mathrm{H}\right\}$ NMR of compound $\mathbf{5 b}\left(75 \mathrm{MHz}\right.$ in $\left.\mathrm{CDCl}_{3}\right)$

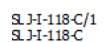

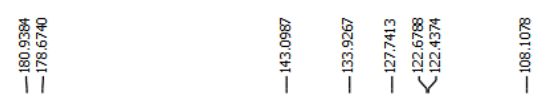

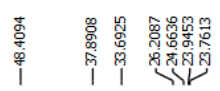

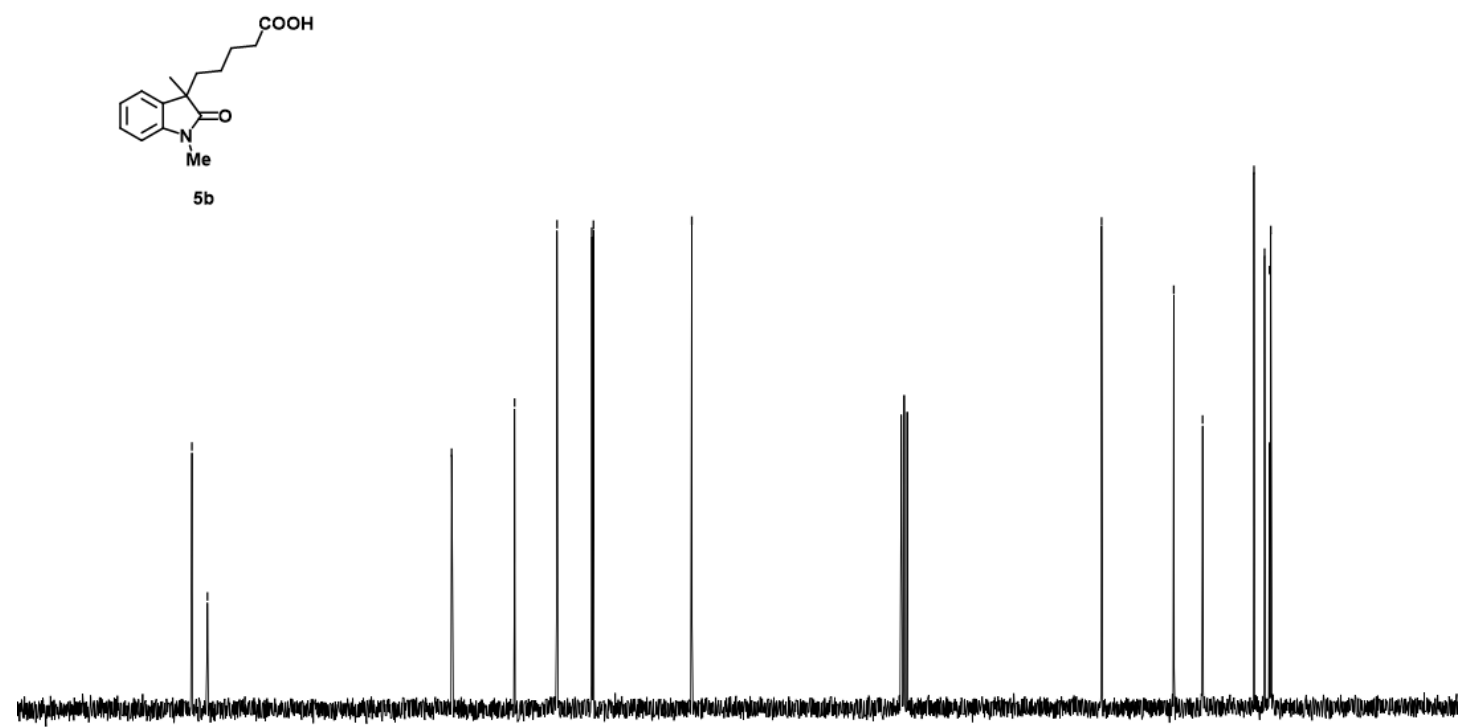

$5 b$

$\stackrel{\circ}{\stackrel{0}{0}}$

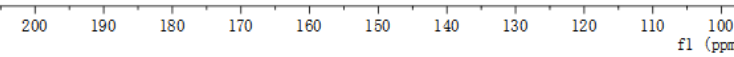


${ }^{1} \mathbf{H}$ NMR of compound $5 \mathbf{c}\left(300 \mathrm{MHz}\right.$ in $\left.\mathrm{CDCl}_{3}\right)$

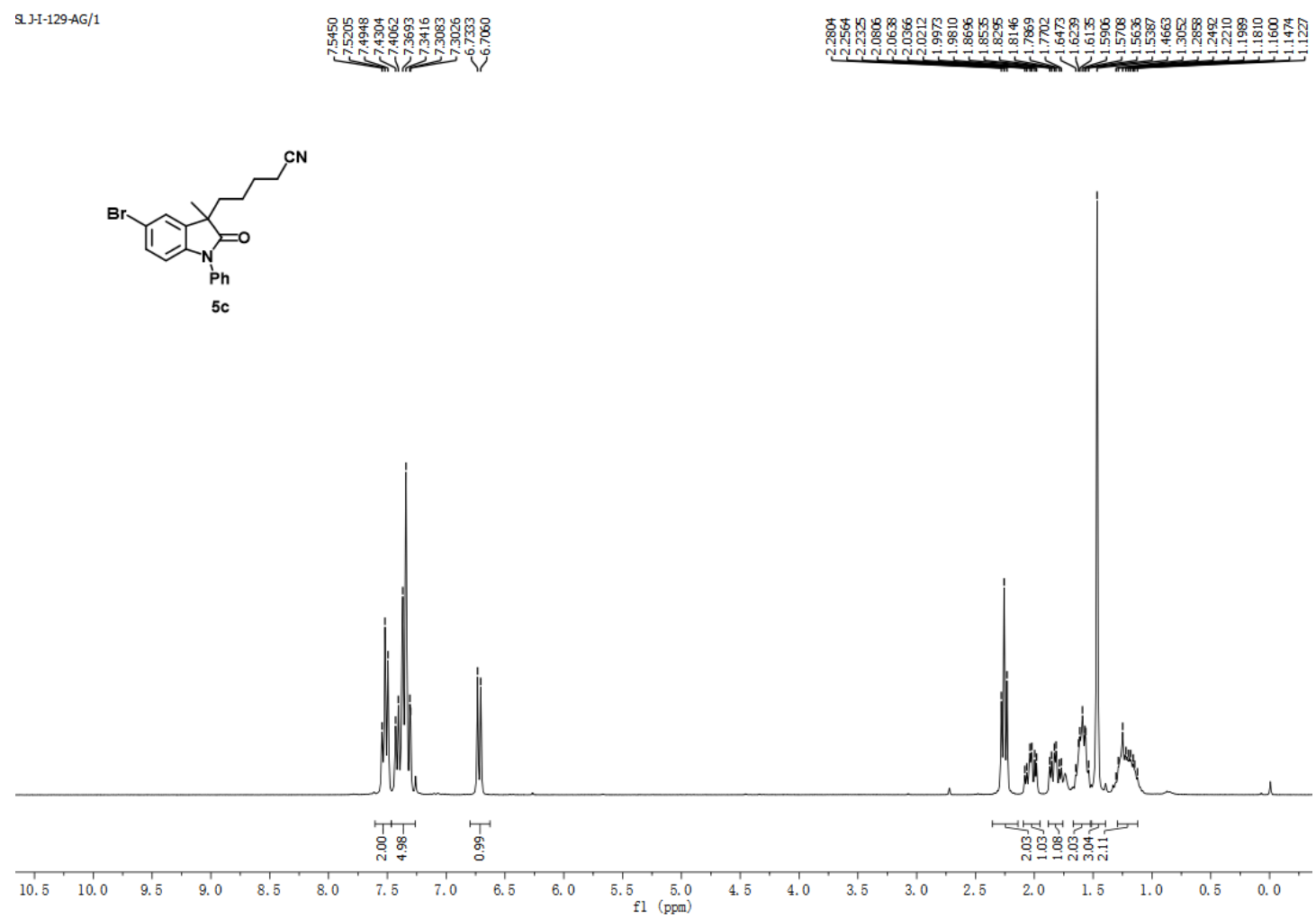

${ }^{13} \mathrm{C}\left\{{ }^{1} \mathrm{H}\right\}$ NMR of compound $5 \mathrm{c}\left(75 \mathrm{MHz}\right.$ in $\left.\mathrm{CDCl}_{3}\right)$
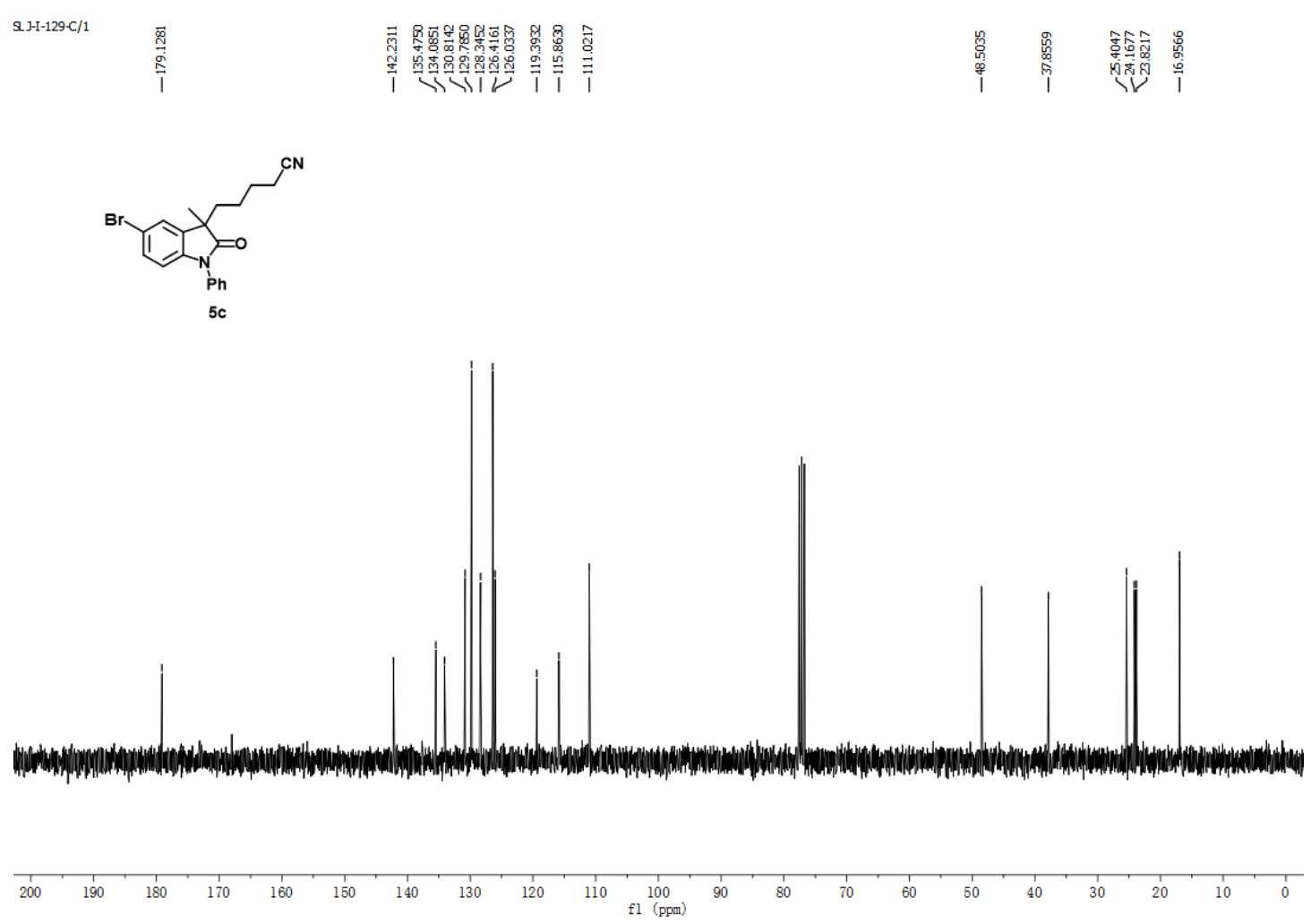